\title{
Molecular Dynamics simulations of polymer liquids on substrates of different topography
}

\author{
Dissertation \\ zur Erlangung des mathematisch-naturwissenschaftlichen Doktorgrades \\ "Doctor rerum naturalium" \\ der Georg-August-Universität Göttingen \\ im Promotionsprogramm ProPhys \\ der Georg-August-University School of Science (GAUSS) \\ vorgelegt von \\ Nikita Tretyakov \\ aus Kaliningrad, Moskauer Gebiet, UdSSR
}

Göttingen, 2012 
Betreuungsausschuss

Prof. Dr. Marcus Müller, Institut für Theoretische Physik

Prof. Dr. Reiner Kree, Institut für Theoretische Physik

Prof. Dr. Stephan Herminghaus, Max-Planck-Institut für Dynamik und

Selbstorganisation, Abtl. Dynamik Komplexer Fluide

Mitglieder der Prüfungskomission

Referent: Prof. Dr. Marcus Müller, Institut für Theoretische Physik

Korreferent: Prof. Dr. Reiner Kree, Institut für Theoretische Physik

Weitere Mitglieder der Prüfungskommission:

Prof. Dr. Annette Zippelius, Institut für Theoretische Physik

Prof. Dr. Jörg Enderlein, III. Physikalisches Institut

Dr. Richard Vink, Institut für Theoretische Physik

Tag der mündlichen Prüfung: 
To my parents

you're always with me 


\section{ACKNOWLEDGEMENTS}

If you ever need anything, please don't hesitate to ask someone else first.

Kurt Cobain

This research was carried out at the Institute for Theoretical Physics at the University of Göttingen from March 2009 until November 2012. At this place, I would like to thank all the people who helped me on the way. Without you, I would not write this thesis or would have done it in a different way (and different means "not better").

First, I thank my supervisor, Prof. Marcus Müller, who was patient enough and let me do all the mistakes I had to make. Without them I would not have learned how to proceed. Then, I thank Prof. Uwe Thiele, who was hospitable enough (4 month in total) at the University of Loughborough (UK). These stays resulted in the end in the joined paper of myself, Prof. Marcus Müller, Prof. Uwe Thiele and another early-stage researcher from Loughborough, Desislava Todorova.

Since research is a process and this process never ends (at least, it lasts till the moment one says: "I quit"), I am indebted to Fabien Léonforte and André Galuschko for active discussions, arguments and guidance. Also I thank André particularly for being to me a lighthouse that shed light on GPU computations.

Scientific work is not possible without filling in applications for trips, solving problems with the residence permit and many other things. I thank our secretaries: Gabriele Schubert, Katrin Glormann and Doris Thies for their assistance in the administrative life. Of course, I thank my colleagues: Kostas Daoulas, Yuliya Smirnova, Ulrich Welling, Giovanni Marelli, Veronica Chappa, Claudio Pastorino and Birger Steinmüller. You were always ready to talk about science and not only science, and I appreciate it. For the permanent reminders that life is not only work I am grateful to my girlfriend Julia. I can't thank you enough. 
I should not forget to mention the funding provided by Marie Curie ITN "MULTIFLOW" (principle organizer - Prof. Uwe Thiele). Also, since the main part of this work deals with computer simulations, I thank the computational centers, where this research was carried out: GWDG Göttingen, HLRN Hanover and GPU-cluster at the Institute for Theoretical Physics.

In the end, I thank Fabien Léonforte, André Galuschko and Prof. Marcus Müller again. They have tried to cultivate in me THE correct articles' grammar. As you can see, your attempts were unsuccessful. 


\section{ABSTRACT}

The properties of polymer films and droplets at substrates of different topography are studied employing particle-based simulation techniques (Molecular Dynamics). The liquid is modeled by short coarse-grained polymer chains of 10 monomers, while the temperature of the system is controlled by the Dissipative Particle Dynamics (DPD) thermostat that conserves momentum locally and provides correct hydrodynamics. Throughout this dissertation we show that macroscopic concepts cannot be straightforwardly extrapolated down to microscopic systems.

At first the flat topography of the substrate is studied. A parameter-passing technique is explored that bridges particle-based MD simulations with continuum descriptions (CD) of the liquid. In particular, the liquid-vapor, solid-liquid and solid-vapor interfacial tensions, and the interface potential are determined by MD simulations. This information is then introduced into continuum models accounting for (i) the full curvature and (ii) a long-wavelength approximation of the curvature (thin film model). A comparison of the dependence of the contact angle on droplet size indicates that the theories agree well if the contact angles are defined in a compatible manner.

Then, we proceed with substrates that are structured symmetrically. Their surface represents a regular array of grooves. The crucial feature of this system is that the typical dimensions of corrugations are of the order of ten diameters of fluid particles. We investigate the influence of corrugation, wettability and pressure on slippage and friction at the solid-liquid interface. For symmetrically structured substrates we observe a gradual crossover between the Wenzel state, where the liquid fills the grooves, and the Cassie state, where the corrugation supports the liquid and the grooves are filled with vapor. Using two independent flow set-ups, we characterize the near-surface flow by the slip length, $\delta$, and the position, $z_{\mathrm{h}}$, at which viscous and frictional stresses are balanced according to Navier's partial slip boundary condition. This hydrodynamic boundary position depends on the pressure inside the channel and may be located above the corrugated surface. In the Cassie state, we observe that the edges of the corrugation contribute to the friction. 
Finally, we consider asymmetrically structured substrates. This type of topography implies an asymmetric response of the droplet's shape onto periodic vibrations. Hence, directed motion of droplets can be achieved. By an analytical phenomenological model we explain the direction of motion and verify it by several computations. Then, the mechanism of the driving is investigated: along with the commonly described motion due to contact lines, we find that the contact area itself may additionally drive the droplet. We show that modifying the roughness of the substrate, one controls the dissipations of the input power due to substrate vibrations and different regimes of droplet motion may be established. 


\section{CONTENTS}

1 Introduction 1

2 Computational models $\quad 5$

2.1 Coarse-grained polymer chain and substrate models . . . . . . . . . . . 5

2.2 Interactions between components of the system $\ldots \ldots \ldots$

2.3 DPD thermostat $\ldots \ldots \ldots \ldots \ldots \ldots$

2.4 Time intergation algorithm $\ldots \ldots \ldots \ldots \ldots$

3 Flat substrates $\quad 11$

3.1 Static properties of thick polymer liquids . . . . . . . . . . . . . . . 11

3.1.1 Virial pressure for a liquid film on a solid substrate . . . . . . 12

3.1.2 Solid-liquid and liquid-vapor interfacial tensions . . . . . . . 16

3.1.3 Adhesion energy and wetting transition . . . . . . . . . . . 17

3.1.4 Solid-vapor interfacial tension . . . . . . . . . . . 18

3.1.5 Interface potential and Derjaguin pressure . . . . . . . . 21

3.2 Static properties of polymer liquids at partial wetting . . . . . . . . 26

3.2.1 Drops as described by continuum description (CD) . . . . . . 28

3.2.2 Contact angles of droplets in MD and CD. Equilibrium contact angle ........................... 31

3.2.3 Comparison of profiles of droplets obtained by CD and particlebased model . . . . . . . . . . . . . . . . . . . . . 36

3.3 Hydrodynamic properties of non-equilibrium polymer liquids . . . . . . 38

3.3.1 Navier's boundary condition . . . . . . . . . . . . . . . . 39 
3.3.2 Hydrodynamic boundary position and slip length . . . . . . . . 42

3.4 Discussion . . . . . . . . . . . . . . . . . . 44

$\begin{array}{lll}4 & \text { Symmetrically structured substrates } & 46\end{array}$

4.1 Static and dynamic properties of polymer droplets . . . . . . . . . . . . 47

4.1 .1 Cassie and Wenzel states . . . . . . . . . . . . . . . . . . . . 49

4.1.2 Energetic barrier and constraint force . . . . . . . . . . . . 51

4.1.3 Velocity profiles of droplets moving under a body force . . . . . . 53

4.2 Correlation between hydrodynamic properties of polymer liquid and surface topography . . . . . . . . . . . . . . . . . . 56

4.2.1 Transition from Cassie to Wenzel states . . . . . . . . . . . . 57

4.2.2 Normal pressure in the bulk phase . . . . . . . . . . . . . . . 60

4.2.3 Comparison of hydrodynamic boundary positions . . . . . . . 62

4.2.4 Comparison of slip length and surface friction . . . . . . . . 65

4.2.5 Pressure-driven flow in patterned channels . . . . . . . . . . . . 68

4.3 Discussion . . . . . . . . . . . . . . . . . 71

$\begin{array}{lll}5 & \text { Asymmetrically structured substrates } & 73\end{array}$

5.1 Directed transport of drops on agitated superhydrophobic substrates . . 74

5.1 .1 Agitated systems . . . . . . . . . . . . . . . . . 74

5.1 .2 Response of a drop on agitation $\ldots \ldots \ldots 76$

5.1.3 Mechanisms of droplet motion . . . . . . . . . . . . . . . . 81

5.1.4 Character of motion. On the way to sort out the dissipations . . . 83

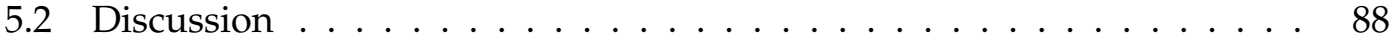

6 Discussion and outlook $\quad 89$

$\begin{array}{lll}\text { Appendix A Properties of the liquid } & 93\end{array}$

$\begin{array}{lr}\text { Curriculum Vitae } & 95\end{array}$

$\begin{array}{ll}\text { List of publications } & 96\end{array}$

$\begin{array}{lr}\text { References } & 97\end{array}$ 


\section{CHAPTER 1}

\section{INTRODUCTION}

God made the bulk; surfaces were invented by the devil.

Wolfgang Pauli

Liquid motion has enchanted many generations of philosophers, scientists and engineers. Probably, one of the first individual attempts to understand liquid motion was made by Archimedes (3rd century BC) and led to the comprehension of the stability of floating bodies and buoyancy. The next great impact to the field, initiating the continuous development of the fluid mechanics and hydrodynamics, was provided by Leonardo da Vinci in 15th century AD [1]. His ingenious sketches, installations and experiments contributed to the continuum principle and visualization of the flows in the presence of obstacles as depicted in Fig. 1.1a.

The other major contribution in understanding properties of a liquid was connected with wetting as shown in Fig. 1.1b. Thomas Young, who obtained the doctoral degree in Göttingen in 1796, described a balance of the forces acting on the contact line of the droplet, lying on a plane solid surface, in equilibrium with its vapor. This macroscopic equation relates the equilibrium contact angle $\theta_{\mathrm{E}}$ of the drop and interfacial tensions between the vapor (V), liquid (L) and solid (S) phases [2]:

$$
\gamma_{\mathrm{SL}}+\gamma \cos \theta_{\mathrm{E}}-\gamma_{\mathrm{SV}}=0,
$$

where for simplicity we dropped the indexes of the liquid-vapor surface tension.

These days, the main trend in liquid mechanics is connected with progressing miniaturization of devices. One can point out the following advantages of scaling down the everyday macroscopic to micro- or even nanoscopic setups [3, 4]: reduced amount of required sample; possibility to create compact and portable integrated devices; low production costs and fast production cycles.

Driven mainly by technological applications [3], microfluidics pursues its objective in development of entire bio- or chemical laboratories on the surface of silica [5] or polymer, usually poly(dimethylsiloxane) or PDMS, chips [6]. In microelectronics, this is 


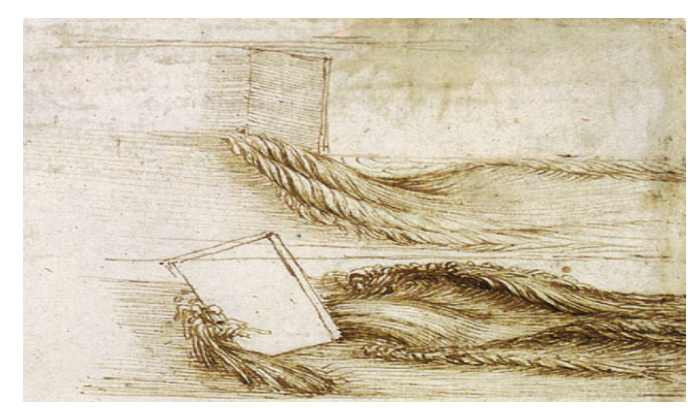

(a)

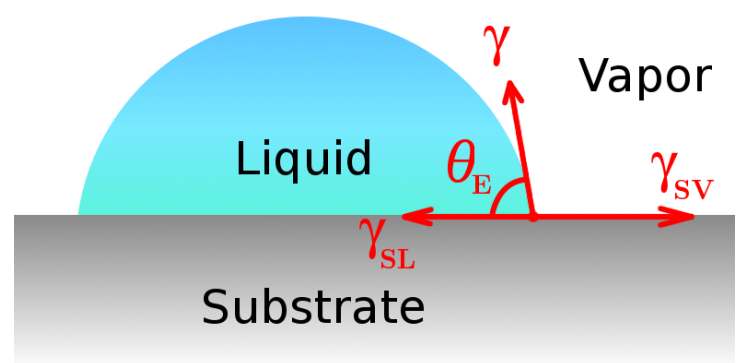

(b)

Figure 1.1: (a) Shown is a part from the Codex Leicester by Leonardo da Vinci with a sketch "Studies of water passing obstacles and falling" (ca. 1508). (b) An illustration of the Young equation, connecting interfacial tensions between phases and the contact angle of the droplet, resting at equilibrium on a flat substrate.

already the case [7], and plenty of devices for biological purposes are developed, e.g. a chemostat used to study the growth of microbial populations [8] or inexpensive diagnostic devices used to carry out sandwich immunoassays [9] as shown in Fig. 1.2.

As the miniaturization continues, nanofluidics separates into an independent scientific field [10]. Without a doubt, the interest to micro- and nanofluidics will persist in the future. To create sophisticated small devices on the basis of fluids, one needs to understand how the macroscopic laws are modified when applied to the microand nanoscopic amount of liquid, when the surface to volume ratio is dramatically large [11]. Therefore, the surface forces (for example, friction and surface tension) are dominating over volume forces (e.g. gravity and inertia) and the importance of the boundaries (e.g. Navier slip condition) increases. The purpose of this work is to provide a detailed (if possible) explanation of the properties and behavior of a small amount of liquid on different kinds of substrates and of the nanoscopic flows in confined geometries.

Since the aim is to study microscopic systems, preserving internal degrees of freedom of the liquid, particle-based Molecular Dynamics (MD) methods are employed. However, as we want to draw an analogy to processes, taking place in micro- and nanofluidic devices, the corresponding length and time scales should be reached. While atomistic MD demands too many computational resources to accomplish this task, we employ a coarse-graining technique, fusing several atoms into a single effective interaction cite.

The following simplification of our physical model is a neglection of evaporating effects, as they are often undesired in many lab-on-a-chip devices. To this end, the liquid constitutes of short polymer chains, providing the density of the vapor phase to be $10^{5}$ times smaller, than the one of the liquid. 


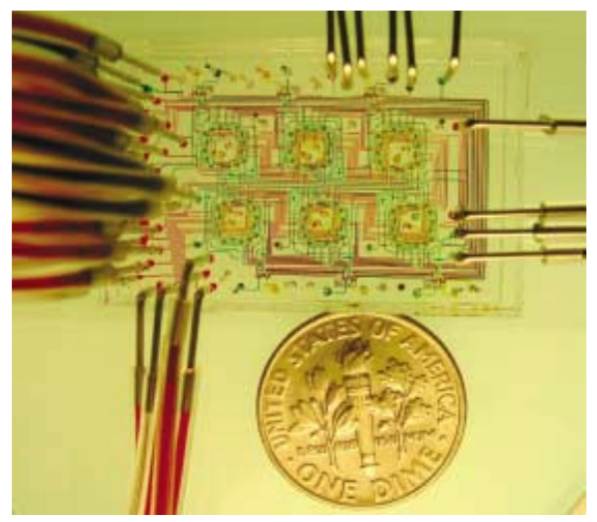

(a)

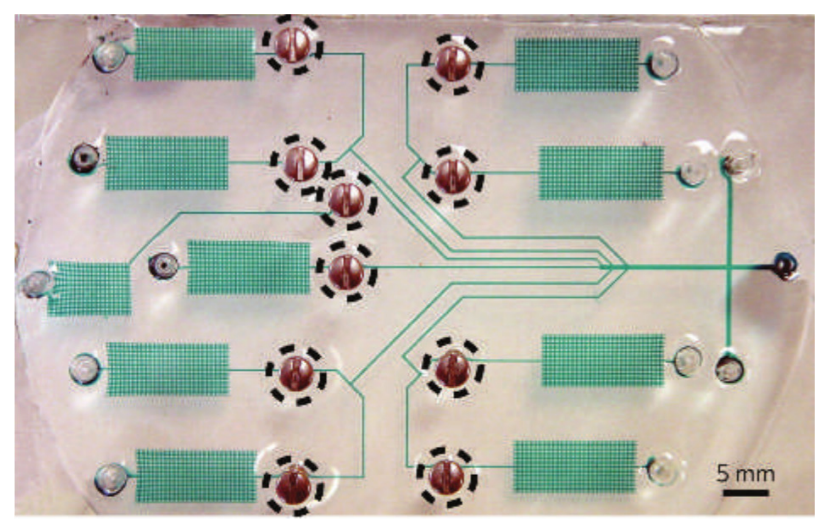

(b)

Figure 1.2: Shown are (a) a microfluidic chemostat to study microbial populations from Ref. [8] and (b) a device performing sandwich immunoassays from Ref. [9]. The microchannels are marked by a color liquid.

The red line through the dissertation is the topography of the substrate. Starting with the simplest one, flat substrate in Chapter 3, we move on with symmetrically structured one in Chapter 4 and finish with the most complex, asymmetrically structured substrate in Chapter 5.

Since the properties and underlying physical processes are very different for various topographies of the substrate, we decided to discuss them in the introductions to specific chapters. On the other hand, the computational model, common for all simulations, is described in Chapter 2. Every chapter ends with a short summary, allowing the reader to address and overview the phenomena he is interested in.

The chapter on flat substrates starts with an introduction of wetting parameters of polymer films. Next, we aim to relate the microscopic interactions of the particle-based model with phenomenological material constants of the continuum description (CD) that can directly address engineering time and length scales. Thus, effort has to be devoted to parameter-passing techniques that transfer information from particle-based models to the CD.

To this end, two questions have to be addressed:

(i) which is the relevant information of the particle-based model that needs to be passed into a CD and

(ii) how to extract this information from the particle-based description?

Then, both MD and CD approaches are used to determine the equilibrium contact angle of a droplet as a function of the size of the droplet. Finally, we compare the profiles of drops of varying size obtained by means of $C D$ and MD simulations. 
We finish Chapter 3 with an overview of the dynamic properties of Poiseuille and Couette flows between flat semi-infinite substrates.

Investigation of properties of liquids at symmetrically structured substrates is discussed in Chapter 4. At first, we present macroscopical theoretical Cassie and Wenzel models of the droplets on structured substrates and show their difference in terms of energetic barriers. Then, we study dynamics of the droplets pushed over structured superhydrophobic substrates by external body forces and present velocity profiles of such droplets. At the end of the chapter, we discuss flows in nanoscopic channels, when the spatial scale of the roughness is of the order of substrate atoms, and the roughness is, therefore, an intrinsic property of the substrate, as interactions and geometry can not be distinguished anymore.

At last, we look at the behavior of nanodroplets at asymmetrically structured substrates in Chapter 5. By agitation of the substrate in a harmonic manner we study a directed motion of droplets. The response of the shape of the drop onto varying vibration frequencies is asymmetric, providing the movement of the center of mass of the droplet. We discuss in detail the mechanisms that may be responsible for the motion.

The simulations of the droplets on asymmetrically structured substrates are performed on GPU facilities using HOOMD Software [12-14], while the liquids at other topographies are investigated by means of self-written programs and analyzing tools. Snapshots of the systems are made in VMD [15] and rendered by Tachyon ray tracing library [16]. 


\title{
COMPUTATIONAL MODELS
}

\author{
That's been one of my mantras - \\ focus and simplicity. Simple can be \\ harder than complex: You have to \\ work hard to get your thinking clean \\ to make it simple. But it's worth it in \\ the end because once you get there, \\ you can move mountains.
}

Steve Jobs

In this section we discuss the relation between atomistic and coarse-grained simulations and present key components of our study - a model for a complex liquid and a substrate. Furthermore, we introduce the interactions between the constituents of the system and a technique to maintain the temperature during the simulations.

\subsection{Coarse-grained polymer chain and substrate models}

Computer simulations always provide a compromise between the speed of calculation, accuracy and degree of realism of the computation model. Atomistic MD simulations use chemically realistic models that preserve an information about positions and velocities of atoms and constraints (e.g. bond length, angles, torsions of the carbonic backbone) and utilize specific force fields like CHARMM [17, 18], OPLS [19] or AMBER [20] to mimic interactions between components of the system. The timestep is relatively small (of the order of $5 \mathrm{ps}=5 \cdot 10^{-15} \mathrm{~s}$ ) and allows one to take into account thermal fluctuations of individual atoms. The length of the trajectory usually does not exceed $100 \mathrm{~ns}$.

To facilitate the study of larger time and length scales, one lumps together a small number of atoms into an effective interaction center (called 'bead') in a coarse-grained model reducing the number of degrees of freedom of the system [21-24, 24, 25]. The advantage of particle-based simulations consists in the ability to refine the model to- 


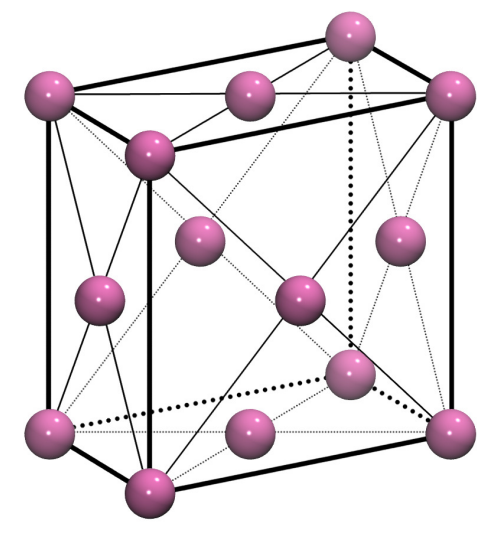

(a)

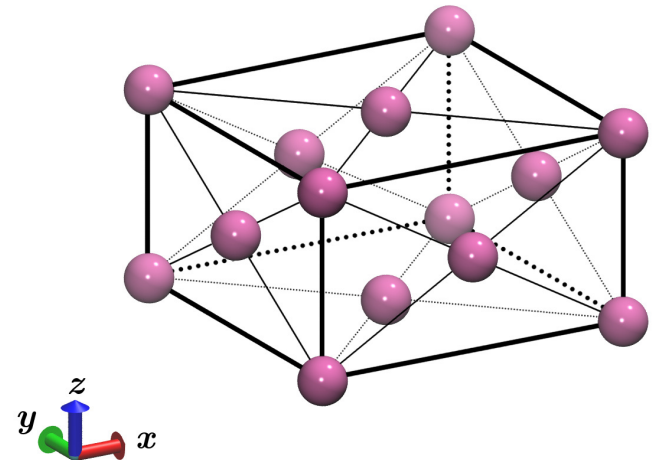

(b)

Figure 2.1: Shown are an original unit cell used in flat substrate simulations $X Y Z$ (a) and the rotated one $X Y z$ (b) used in simulations with structured substrates. The number density of the substrate atoms is constant $\rho_{\mathrm{s}}=2.67 \sigma^{-3}$ in all performed simulations.

wards a chemically realistic description and to include effects of thermal fluctuations and of discreteness of matter, that are expected to become important on small length scales.

In the present work, we employ a widely used coarse-grained model of a polymer chain of $N_{p}=10$ monomers [21, 26, 27]. The polymeric liquid is placed onto a supporting substrate that is not smooth and homogeneous [26, 28], but is composed of particles (called 'atoms') arranged in a face-centered-orthorhombic ( $f c o$ ) lattice. The unit cell of the lattice consists of four atoms ( 8 atoms in the corners are shared by 8 cells and 6 atoms on the faces by two cells, i.e. $8 / 8+6 / 2=4$ atoms) and occupies a volume of $a_{\mathrm{s}} \cdot \sqrt{3} a_{\mathrm{s}} \cdot \sqrt{3} a_{\mathrm{s}}=3 a_{\mathrm{s}}^{3}$, where $a_{\mathrm{s}}=\sqrt[3]{0.5} \sigma$. The number density is therefore $\rho_{\mathrm{s}}=$ $2.67 \sigma^{-3}$.

Despite being similar in linear sizes of unit cells, the substrates in use differ in topography. In Chapter 3, a flat substrate is composed of two atomic layers of $f c o$ lattice. Later, in Chapters 4 and 5, the orientation of the unit cell is changed and this new cell is used to construct structured substrates. The original $X y Z$ cell is rotated by $90^{\circ}$ around $x$-axis and is referred to as a $X Y z$ unit cell. The transformation is shown in Fig. 2.1. The notations of the cells represent the relations between the lattice vectors $\vec{x}, \vec{y}$ and $\vec{z}$.

It is shown in Sec. 4.2.4 that the change of the orientation of the unit cell of a flat substrate dramatically influences friction at the liquid-substrate interface. This effect originates from the fact, that in $x y$-plane the atomic layer composed of $X y Z$ cells is more densely packed and, therefore, the isopotential surface is more uniform than the layer of $X Y z$ cells (cf. top faces of the cells in Fig. 2.1). 


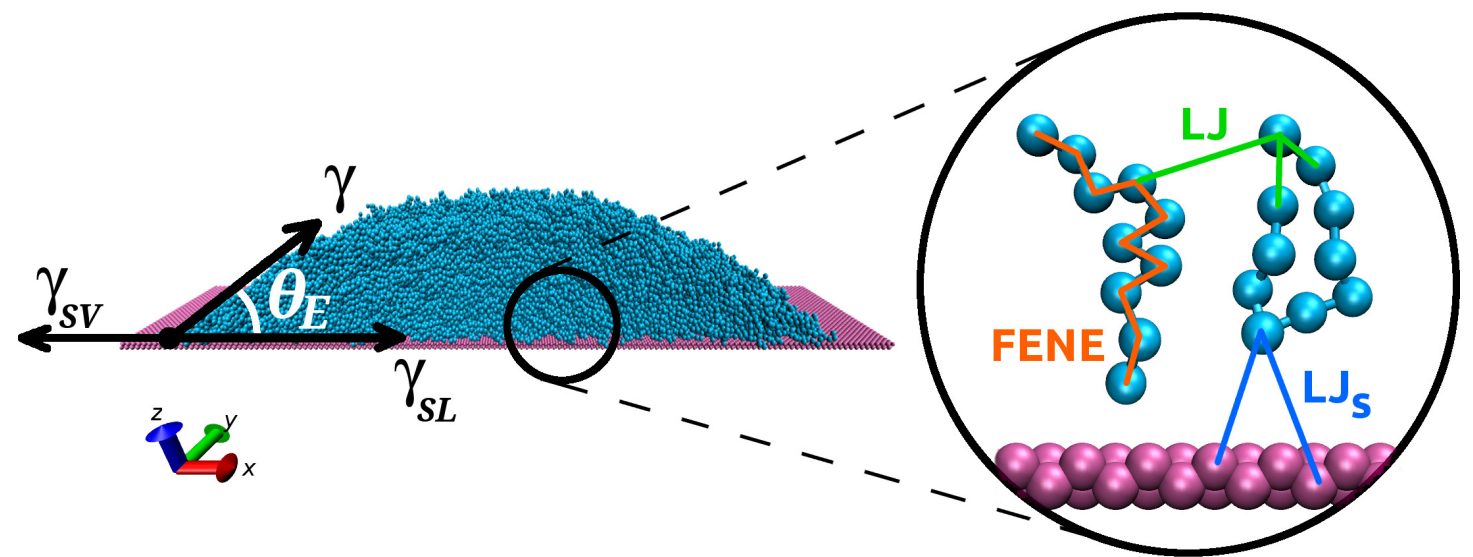

Figure 2.2: Snapshot from MD simulation of a cylindrical drop on a flat substrate with illustration of Young's equation (left). The enlargement close to the substrate (right) sketches the pairwise bead potentials. Coarse-grained beads of polymer chains (blue) interact with each other and with the substrate modeled by two atomic layers of face-centered-orthorhombic lattice (lilac)

\subsection{Interactions between components of the system}

The interaction potentials of the model are sketched in Fig. 2.2. All bonded and nonbonded beads interact via truncated and shifted Lennard-Jones (LJ) potentials

$$
U\left(r_{i j}\right)=U_{\mathrm{LJ}}\left(r_{i j}\right)-U_{\mathrm{LJ}}\left(r_{c}\right)
$$

with

$$
U_{\mathrm{LJ}}\left(r_{i j}\right)=4 \epsilon\left[\left(\frac{\sigma}{r_{i j}}\right)^{12}-\left(\frac{\sigma}{r_{i j}}\right)^{6}\right]
$$

if the distance $r_{i j}=\left|\vec{r}_{i}-\vec{r}_{j}\right|$ between $i$ and $j$ particles is smaller than the cutoff distance $r_{c}=2 \times 2^{1 / 6} \sigma . U_{\mathrm{LJ}}\left(r_{c}\right)$ is the LJ potential evaluated at the cutoff distance. All LJ parameters are set to unity, $\epsilon=1$ and $\sigma=1$, i.e. we express all energies and lengths in units of $\epsilon$ and $\sigma$, respectively. Furthermore, all beads have unit mass $m=1$. The reduced time unit $\tau$ is set by a combination of the LJ parameters as $\tau=\sigma \sqrt{\frac{m}{\epsilon}}$.

The individual beads are connected into chains employing a finite extensible nonlinear elastic (FENE) potential given by $[29,30]$

$$
U_{\mathrm{FENE}}=\left\{\begin{array}{cc}
-\frac{1}{2} k R_{0}^{2} \ln \left[1-\left(\frac{r_{i j}}{R_{0}}\right)^{2}\right] & \text { for } r_{i j}<R_{0} \\
\infty & \text { for } r_{i j} \geq R_{0}
\end{array}\right.
$$

where $R_{0}=1.5 \sigma$ and $k=30 \epsilon / \sigma^{2}$.

We also employ a truncated and shifted LJ interaction between the beads of the liquid and the individual constituents of the substrate

$$
U^{\mathrm{s}}\left(r_{i j}\right)=U_{\mathrm{LJ}}^{\mathrm{s}}\left(r_{i j}\right)-U_{\mathrm{LJ}}^{\mathrm{s}}\left(r_{c}\right)
$$


with

$$
U_{\mathrm{LJ}}^{s}=4 \epsilon_{\mathrm{s}}\left[\left(\frac{\sigma_{\mathrm{s}}}{r_{i j}}\right)^{12}-\left(\frac{\sigma_{\mathrm{s}}}{r_{i j}}\right)^{6}\right]
$$

where the length scale $\sigma_{\mathrm{s}}=0.75 \sigma$. By changing $\epsilon_{\mathrm{s}}$ from $0.2 \epsilon$ to $\epsilon$, one tunes the wettability of the system from non-wetting (polymer droplet with contact angle close to $\theta_{\mathrm{E}}=180^{\circ}$ ) to complete wetting (polymer film with $\theta_{\mathrm{E}}=0^{\circ}$ ).

\subsection{DPD thermostat}

The temperature of the system is controlled by a dissipative particle dynamics (DPD) thermostat [31,32]. In DPD, the total force on a single particle $i$ is given by

$$
F_{\text {tot }}=\sum_{j \neq i}\left(\mathbf{F}_{i j}+\mathbf{F}_{i j}^{\mathrm{D}}+\mathbf{F}_{i j}^{\mathrm{R}}\right)
$$

where the conservative force $\mathbf{F}_{i j}$ is derived from the potentials between particles, $\mathbf{F}_{i j}^{\mathrm{D}}$ is a dissipative force, and $\mathbf{F}_{i j}^{\mathrm{R}}$ is a random force. The dissipative and random forces act on pairs of particles and are of the form

$$
\begin{gathered}
\mathbf{F}_{i j}^{\mathrm{D}}=-\gamma_{\mathrm{DPD}} \omega_{\mathrm{D}}\left(r_{i j}\right)\left(\overrightarrow{e_{i j}} \cdot \overrightarrow{v_{i j}}\right) \overrightarrow{e_{i j}}, \\
\mathbf{F}_{i j}^{\mathrm{R}}=\zeta \omega_{\mathrm{R}}\left(r_{i j}\right) \theta_{i j} \overrightarrow{e_{i j},}
\end{gathered}
$$

where the unit vector $\overrightarrow{i_{i j}}=\overrightarrow{r_{i j}} / r_{i j}$ points from the $j$-th to the $i$-th particle. In order to obey the fluctuation-dissipation theorem, the damping coefficient, $\gamma_{\mathrm{DPD}}$, is connected to the amplitude of the noise, $\zeta$, via the fluctuation-dissipation theorem $\zeta^{2}=2 k_{\mathrm{B}} T \gamma_{\mathrm{DPD}}$, and the weight functions are defined as

$$
\omega_{\mathrm{R}}^{2}\left(r_{i j}\right)=\omega_{\mathrm{D}}\left(r_{i j}\right)=\left\{\begin{array}{cl}
\left(1-\frac{r_{i j}}{r_{c}}\right)^{2} & \text { for } r_{i j}<r_{c} \\
0 & \text { for } r_{i j} \geq r_{c}
\end{array}\right.
$$

We fix $\gamma_{\mathrm{DPD}}=0.5$ throughout the simulations. The term $\theta_{i j}$ in Eq. (2.8) is a random noise term such that $\theta_{i j}=\theta_{j i}$ and its first and second moments are

$$
\begin{gathered}
\left\langle\theta_{i j}\right\rangle=0, \\
\left\langle\theta_{i j}(t) \theta_{k l}\left(t^{\prime}\right)\right\rangle=\left(\delta_{i k} \delta_{j l}+\delta_{i l} \delta_{j k}\right) \delta\left(t-t^{\prime}\right) .
\end{gathered}
$$

We use uniformly distributed random numbers [33] with the first and second moments dictated by the relations above.

Since the dissipative and random forces and, of course, also the conservative forces satisfy Newton's third law, they locally conserve momentum, i.e. they preserve the hydrodynamics of the flow (in contrast to the dissipative macroscopic behavior of Langevin dynamics). Using this DPD thermostat, we maintain the constant temperature, $k_{\mathrm{B}} T=1.2 \epsilon$, where $k_{\mathrm{B}}$ is the Boltzmann constant. 


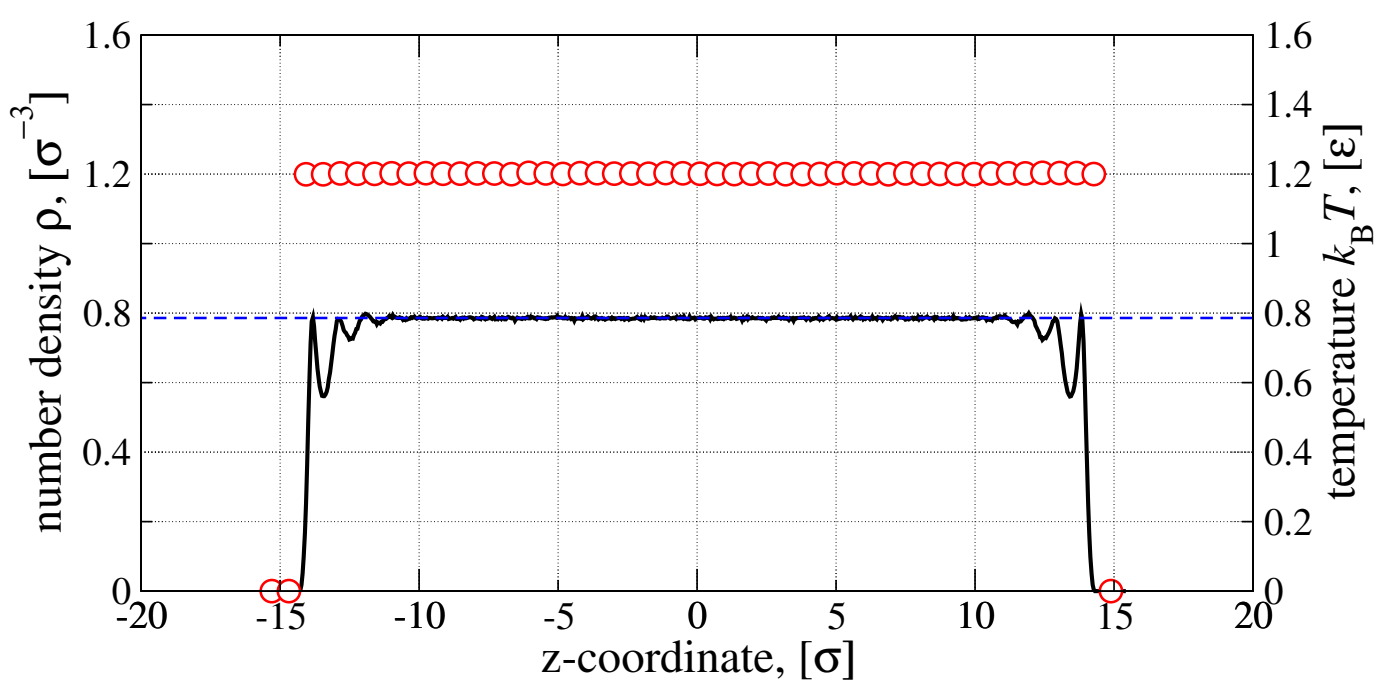

Figure 2.3: Temperature profile (circles) of a polymer film confined between two flat walls with attraction strength $\epsilon_{\mathrm{s}}=0.4 \epsilon$ at coexistence density (dashed horizontal line). The temperature is defined via the kinetic energy. The density profile is plotted by the solid line. Packing of the liquid, characteristic for the models of the liquid and solid, is seen at the interface.

However, although the DPD thermostat does locally conserve momentum, we do not have a momentum conservation of liquid-substrate interactions. The substrate atoms are frozen at their lattice nodes and act on the polymer liquid by uncompensated forces, as opposed to soft substrates [34]. The temperature of such substrate is $T_{\mathrm{s}}=0$, but it does not lead to any noticeable temperature gradient induced in the liquid in close vicinity of the solid, as proved by the temperature profile presented in Fig. 2.3. There, the liquid in the vicinity of the solid has the same temperature as the bulk. Also, a weak local violation of the momentum conservation does not influence the properties of the droplets, as we verified by comparison of drops sitting on two different substrates: the one with frozen atoms and the one with atoms, oscillating around their equilibrium positions (the Einstein crystal).

\subsection{Time intergation algorithm}

The equations of motion (2.6) are discretized by time steps $\Delta t$. For MD simulations a balance between efficiency and accuracy should be found. The former is usually limited by force evaluation, which is the heaviest component of computations, and rules out any Runge-Kutta method [35]. The latter excludes the simplest Euler integration, as it is only a first-order method. A good compromise between efficiency and accuracy is provided by the simple, but robust and reversible Verlet or leap-frog algorithms $[36,37]$.

The basic form of the Verlet algorithm follows immediately from the Taylor expansions 
of the particle coordinates $\vec{r}(t+\Delta t)$ and $\vec{r}(t-\Delta t)$, namely

$$
\vec{r}(t+\Delta t)=2 \vec{r}(t)-\vec{r}(t-\Delta t)+\vec{a}(t) \Delta t^{2}+O\left(\Delta t^{4}\right),
$$

where $\vec{a}(t)$ is the acceleration of the particle at time $t$. The error here is of the order of $\Delta t^{4}$. The problem with this version of the algorithm is that velocities are not explicitly involved into solution (2.12). Instead, one can calculate them via

$$
\vec{v}(t+\Delta t)=\frac{\vec{r}(t+\Delta t)-\vec{r}(t-\Delta t)}{2 \Delta t}+O\left(\Delta t^{2}\right) .
$$

Nevertheless, this method increases the error to the order of $\Delta t^{2}$.

To include velocities explicitly into integration, the leap-frog algorithm may be used. The coordinates and velocities are calculated at integer and integer plus a half time steps, correspondingly (as the name suggests).

However, the most commonly used version of the integration scheme is the one, where the coordinates and velocities are obtained at the same time steps, the Velocity Verlet algorithm [36, 38]:

$$
\begin{aligned}
\vec{r}(t+\Delta t) & =\vec{r}(t)+\vec{v}(t) \Delta t+\frac{1}{2} \vec{a}(t) \Delta t^{2}+O\left(\Delta t^{4}\right), \\
\vec{v}(t+\Delta t) & =\vec{v}(t)+\frac{\vec{a}(t)+\vec{a}(t+\Delta t)}{2} \Delta t+O\left(\Delta t^{2}\right) .
\end{aligned}
$$

The error of the Velocity Verlet algorithm is exactly the same as for basic Verlet scheme. Providing good computation speed and reliability of the Velocity Verlet algorithm, we employ it to integrate equations of motion using a time step $\Delta t=0.005 \tau$. 


\section{FLAT SUBSTRATES}

Do not worry about your difficulties in mathematics. I can assure you that mine are still greater.

Albert Einstein

This Chapter deals with static and dynamic properties of polymer films and droplets on a flat solid substrate. A flat substrate studied here is composed of two atomic layers of $X y Z$ unit cell presented in Sec. 2.1. A side view of the substrate is shown in Fig. 2.2. At first, we consider general wetting properties of thick liquid films (i.e. films that have a bulk region) on supporting solid substrates. Special attention is devoted to the rigorous definition of the wetting transition and the stability of thin films is investigated by calculation of the interface potential.

Then, in the second part of the Chapter, we bridge particle-based MD simulations with a continuum description (CD) of the liquid, suggesting a parameter-passing scheme. The reliability of the method is tested for droplets of various sizes with different contact angles.

Finally, the last part is devoted to the fundamental insight into dynamic properties of liquids confined between flat walls and application of the hydrodynamic boundary condition to describe the solid-liquid interface independently from the type of the flow.

\subsection{Static properties of thick polymer liquids}

In this section we discuss equilibrium properties of polymeric liquid films on a supporting substrate. At first, the individual components of Young's equation that balances surface and interfacial tensions are considered. The technique, allowing for independent calculations of surface (solid-liquid) and interfacial (liquid-vapor) tensions of a thick film, is presented. Then, the wetting transition is localized for our solid-liquid model. After it, we discuss the solid-vapor surface tension. Finally, regions of stable 


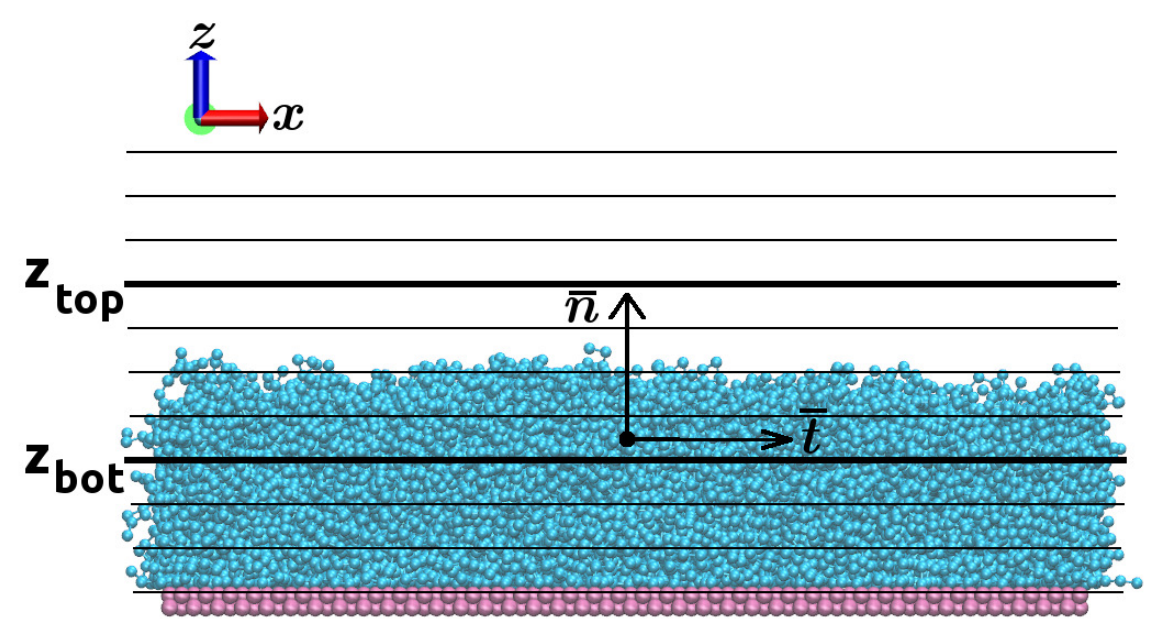

Figure 3.1: Sketch of the slab geometry used to calculate the liquid-vapor interfacial tension $\gamma$. The pressure tensor components $p_{\mathrm{n}}(z)$ and $p_{\mathrm{t}}(z)$ are calculated in every slab $k$ and then their difference is integrated across the interface.

film thickness are determined by means of the interface potential.

\subsubsection{Virial pressure for a liquid film on a solid substrate}

We study a supported thick polymer film as illustrated in Fig. 3.1 in the canonical ensemble. By virtue of the low vapor pressure of the polymer liquid, one can neglect evaporation effects. The flat liquid-vapor interface allows us to divide the system into thin parallel slabs (separated by the horizontal grey lines in Fig. 3.1), whose normal vector $\vec{n}$ is perpendicular to the substrate. All relevant quantities can then be averaged over each slab, resulting in fields that depend on the $z$-coordinate only.

In order to obtain the tension of the liquid-vapor and solid-liquid interfaces, $\gamma$ and $\gamma_{S L}$, we consider a virtual change of the geometry of the simulation box such that the total volume $V$ remains unaltered. Using the scaling parameter $\Lambda$, we relate the new linear dimensions, $L_{x}^{\prime}, L_{y}^{\prime}, L_{z}^{\prime}$ of the simulation box to the original ones via $L_{x}^{\prime}=\sqrt{\Lambda} L_{x}, L_{y}^{\prime}=$ $\sqrt{\Lambda} L_{y}, L_{z}^{\prime}=\frac{1}{\Lambda} L_{z}$. Only the liquid is subjected to this virtual change of the geometry but not the solid support.

The value $\Lambda<1$ corresponds to a lateral squeezing of the liquid film on top of a solid substrate and a concomitant increase of the film thickness $h^{\prime}=\frac{1}{\Lambda} h$, where we have assumed that the liquid is incompressible. In the continuum model such a transformation gives rise to the the following infinitesimal change of the canonical free energy [39]

$$
\left.\frac{\mathrm{d} F_{0}(\Lambda)}{\mathrm{d} \Lambda}\right|_{\Lambda=1}=\left.\left[\gamma_{\mathrm{SL}}+\gamma\right] \frac{\mathrm{d} L_{x}^{\prime} L_{y}^{\prime}}{\mathrm{d} \Lambda}\right|_{\Lambda=1},
$$

where, contrary to the related works in grandcanonical ensemble $[40,41]$, we use the 


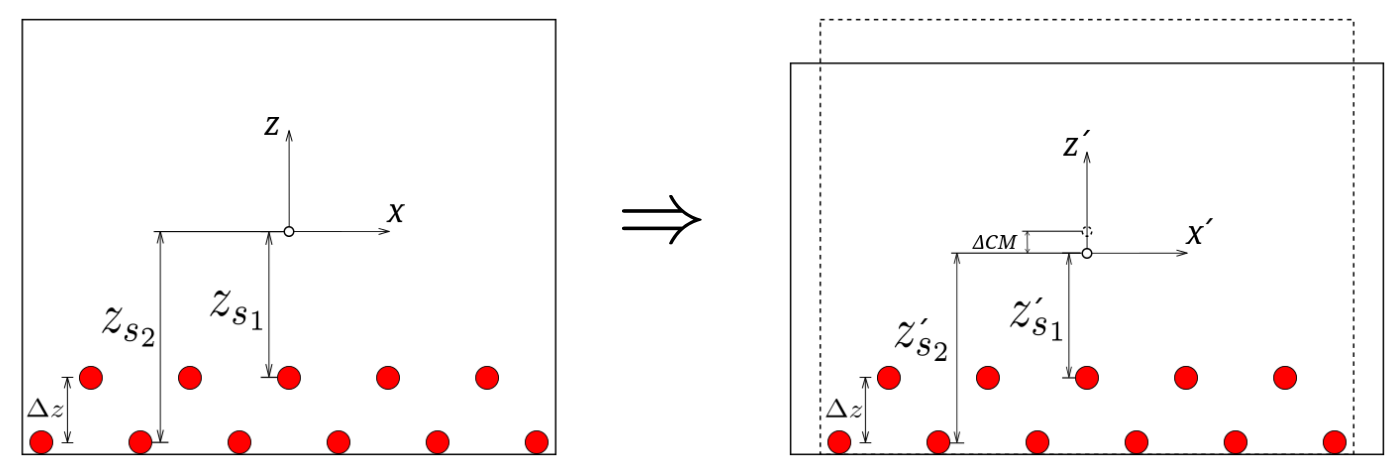

Figure 3.2: Unscaled simulation box (left). Red circles represent two layers of substrate atoms. The origin with $x$ and $z$ axes is in the middle of the box. Scaled simulation box (right). The origin has moved by $\triangle C M$ against $z$ axis, but its lateral coordinates, $x$ and $y$, remain unaltered. $y$ axis is not shown for simplicity.

property of a canonical one and keep the number of particles in the liquid constant, i.e. constant volume $h L_{x} L_{y}=h^{\prime} L_{x}^{\prime} L_{y}^{\prime}$ of the film

$$
\frac{\mathrm{d} L_{x}^{\prime} L_{y}^{\prime}}{L_{x}^{\prime} L_{y}^{\prime}}+\frac{\mathrm{d} h^{\prime}}{h^{\prime}}=0
$$

The scaling affects the beads of the polymeric liquid only, i.e., the lateral coordinates $x$ and $y$ are scaled by the factor $\sqrt{\Lambda}$ and the normal component $z$ is scaled by $1 / \Lambda$. Since the origin of the coordinate system is at the center of the simulation cell, the $z$ coordinates of the first layer of the substrate is also scaled by the factor $1 / \Lambda$ and the second row is shifted such that the distance $\Delta z$ between the two layers of the substrate remains unchanged. The lateral coordinates of the substrate particles are not changed as depicted in Fig. 3.2.

In order to compute the change of free energy, we consider the canonical partition function

$$
\mathcal{Z}=\frac{1}{N ! \lambda_{\mathrm{T}}^{3 N}} \int \prod_{i=1}^{N} d^{3} \vec{r}_{i} \exp \left[-\beta \sum_{i<j} U\left(\vec{r}_{i}-\vec{r}_{j}\right)-\beta \sum_{s, i} U^{\mathrm{s}}\left(\vec{r}_{i}-\vec{r}_{s}\right)\right]
$$

where $N$ is the number of particles in the system, $\beta=\frac{1}{k_{\mathrm{B}} T}$ and $\lambda_{T}$ is the thermal deBroglie wavelength. $U$ denotes the bonded and non-bonded interactions between the polymer beads $i$ and $j$, and $U^{s}$ are the interactions between the polymer beads $i$ and the substrate particles $s$.

This separation of potentials allows us to express the partition function, $\mathcal{Z}(\Lambda)$, of the 
scaled system through the scaling transformation of the original positions

$$
\begin{aligned}
\mathcal{Z}(\Lambda)=\frac{1}{N ! \lambda_{\mathrm{T}}^{3 N}} \int \prod_{i=1}^{N} \mathrm{~d}^{3} \vec{r}_{i} \exp [ & -\beta \sum_{i<j} U\left(\sqrt{\Lambda}\left(x_{i}-x_{j}\right), \sqrt{\Lambda}\left(y_{i}-y_{j}\right), \frac{1}{\Lambda}\left(z_{i}-z_{j}\right)\right) \\
& -\beta \sum_{s_{1}, i} U^{\mathrm{s}}\left(\sqrt{\Lambda} x_{i}-x_{s_{1}}, \sqrt{\Lambda} y_{i}-y_{s_{1}}, \frac{1}{\Lambda} z_{i}-\frac{1}{\Lambda} z_{s_{1}}\right) \\
& \left.-\beta \sum_{s_{2}, i} U^{\mathrm{s}}\left(\sqrt{\Lambda} x_{i}-x_{s_{2}}, \sqrt{\Lambda} y_{i}-y_{s_{2}}, \frac{1}{\Lambda} z_{i}-\left(\frac{1}{\Lambda} z_{s_{1}}-\Delta z\right)\right)\right]
\end{aligned}
$$

where we explicitly separated the interaction of the polymer beads with the first and second layer of the substrate, $z_{s_{1}}^{\prime}=\frac{1}{\Lambda} z_{s_{1}}$ and $z_{s_{2}}^{\prime}=z_{s_{1}}^{\prime}-\Delta z$. Differentiation with respect to $\Lambda$ yields

$$
\begin{aligned}
\left.\frac{\mathrm{d} \mathcal{Z}}{\mathrm{d} \Lambda}\right|_{\Lambda=1}=-\frac{\beta}{N ! \lambda_{\mathrm{T}}^{3 N}} \int \prod_{i=1}^{N} \mathrm{~d}^{3} \vec{r}_{i}\{ & \sum_{i<j}\left(\frac{1}{2}\left(\frac{\partial U}{\partial x_{i j}} x_{i j}+\frac{\partial U}{\partial y_{i j}} y_{i j}\right)-\frac{\partial U}{\partial z_{i j}} z_{i j}\right) \exp \left[-\beta \sum_{i<j} U\right] \quad(3.5) \\
& +\sum_{s_{1}, i}\left(\frac{1}{2}\left(\frac{\partial U^{\mathrm{s}}}{\partial x_{i s_{1}}} x_{i}+\frac{\partial U^{\mathrm{s}}}{\partial y_{i s_{1}}} y_{i}\right)-\frac{\partial U^{\mathrm{s}}}{\partial z_{i s_{1}}} z_{i s_{1}}\right) \exp \left[-\beta \sum_{s_{2}, i} U^{\mathrm{s}}\right] \\
& \left.+\sum_{s_{2}, i}\left(\frac{1}{2}\left(\frac{\partial U^{\mathrm{s}}}{\partial x_{i s_{2}}} x_{i}+\frac{\partial U^{\mathrm{s}}}{\partial y_{i s_{2}}} y_{i}\right)-\frac{\partial U^{\mathrm{s}}}{\partial z_{i s_{2}}} z_{i s_{1}}\right) \exp \left[-\beta \sum_{s_{1}, i} U^{\mathrm{s}}\right]\right\}
\end{aligned}
$$

where $x_{i j}=x_{i}-x_{j}$. Substituting $z_{i s_{1}}=z_{i s_{2}}-\Delta z$, we write the change of the free energy in the form

$$
\begin{aligned}
\left.\frac{\mathrm{d} F_{0}}{\mathrm{~d} \Lambda}\right|_{\Lambda=1}= & -\left.k_{\mathrm{B}} T \frac{1}{\mathcal{Z}} \frac{\mathrm{d} \mathcal{Z}}{\mathrm{d} \Lambda}\right|_{\Lambda=1} \\
= & \left\langle\sum_{i<j}\left(\frac{1}{2}\left(\frac{\partial U}{\partial x_{i j}} x_{i j}+\frac{\partial U}{\partial y_{i j}} y_{i j}\right)-\frac{\partial U}{\partial z_{i j}} z_{i j}\right)\right\rangle \\
& +\left\langle\sum_{s, i}\left(\frac{1}{2}\left(\frac{\partial U^{\mathrm{s}}}{\partial x_{i s}} x_{i}+\frac{\partial U^{\mathrm{s}}}{\partial y_{i s}} y_{i}\right)-\frac{\partial U^{\mathrm{s}}}{\partial z_{i s}} z_{i s}\right)\right\rangle+\left\langle\sum_{s_{2}, i} \frac{\partial U^{\mathrm{s}}}{\partial z_{i s_{2}}}\right\rangle \Delta z \\
= & \left\langle\sum_{i<j}\left(f_{z, i j} z_{i j}-\frac{1}{2}\left(f_{x, i j} x_{i j}+f_{y, i j} y_{i j}\right)\right)\right\rangle \\
& +\left\langle\sum_{s, i}\left(f_{z, i s}^{\mathrm{s}} z_{i s}-\frac{1}{2}\left(f_{x, i s}^{\mathrm{s}} x_{i}+f_{y, i s}^{\mathrm{s}} y_{i}\right)\right)-\sum_{s_{2}, i} f_{z, i s_{2}}^{\mathrm{s}} \Delta z\right\rangle
\end{aligned}
$$

where $f_{x, i j}$ denotes the $x$-component of the force acting between polymer beads, $i$ and $j .\langle\cdots\rangle$ denote averages in the canonical ensemble.

The first term of Eq. (3.8) is the anisotropy of the pressure inside the liquid [42, 43]. Using the approach of Irving and Kirkwood [44] we define profiles of the normal and tangential pressure in a slab $k$ according to

$$
p_{\mathrm{n}}(k)=k_{\mathrm{B}} T\langle\rho(k)\rangle+\frac{1}{V_{\mathrm{sl}}}\left\langle\sum_{i<j}^{(k)} f_{z, i j} z_{i j} \eta_{k}\left(\vec{r}_{i j}\right)\right\rangle,
$$

and

$$
p_{\mathrm{t}}(k)=k_{\mathrm{B}} T\langle\rho(k)\rangle+\frac{1}{2 V_{\mathrm{sl}}}\left\langle\sum_{i<j}^{(k)}\left(f_{x, i j} x_{i j}+f_{y, i j} y_{i j}\right) \eta_{k}\left(\vec{r}_{i j}\right)\right\rangle
$$


32832 atoms, 2280 chains, box $(52.24 \times 52.38 \times 24.60) \sigma, T=1.2 \varepsilon$, after 700000 steps

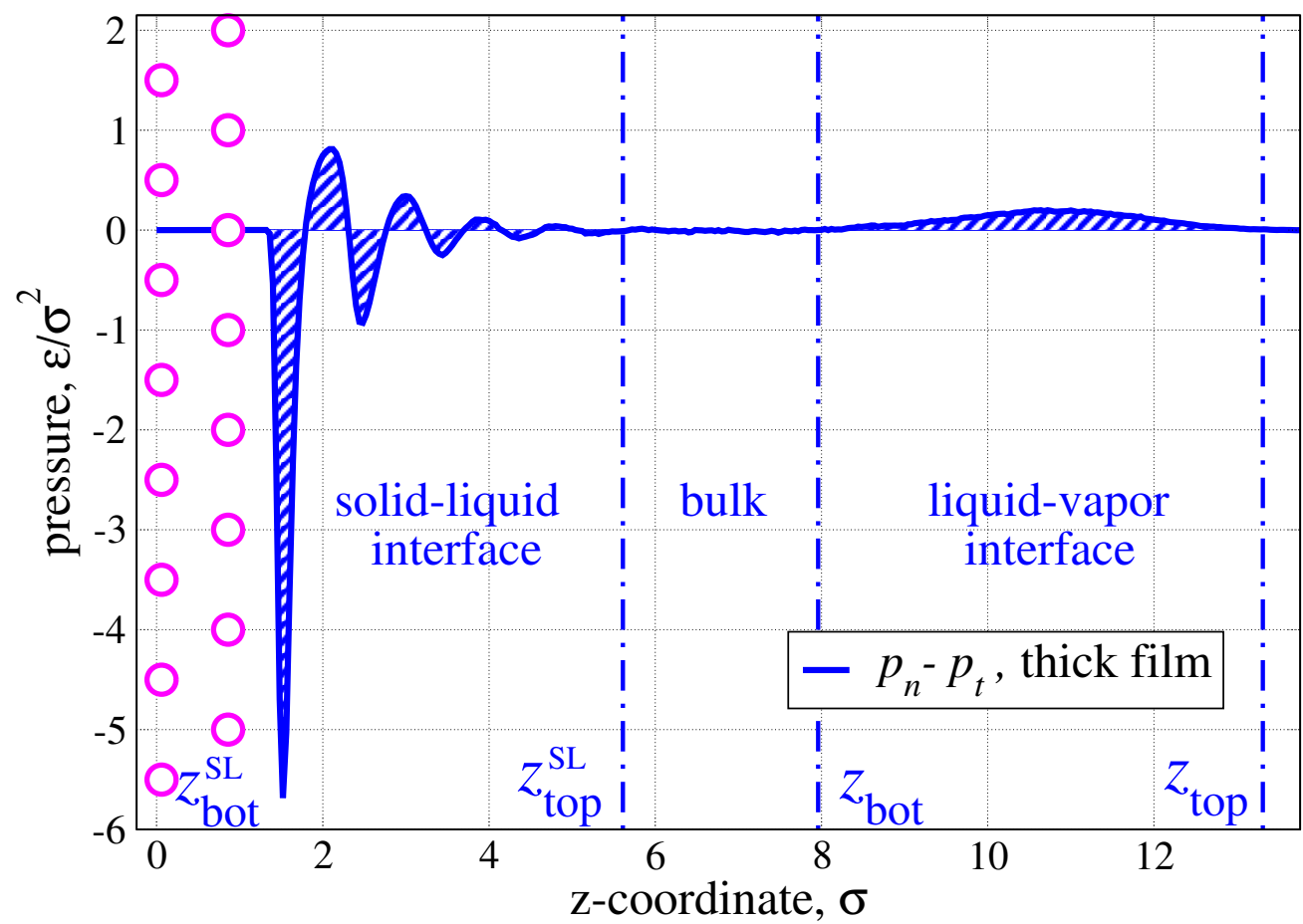

Figure 3.3: A typical diagram of normal and tangential pressure differences along the $z$ axis in a thick film is plotted. Two atomic layers of the flat substrate are represented as circles. The presence of the bulk phase in the middle of the film allows one to separate pressure differences associated with surface (solid-liquid) or interfacial (liquid-vapor) tensions.

where $\rho(k)$ is the number density in a slab $k$ and $V_{\mathrm{sl}}$ denotes the volume of the slab. The sum $\sum_{i<j}^{(k)}$ runs over particles $i$ and $j$ if the line connecting them crosses the boundary of slab $k$ (then $\eta_{k}\left(\vec{r}_{i j}\right)$ is the fraction of that line that is located in slab $k$ ) or if both particles are in slab $k$ (then $\eta_{k}\left(\vec{r}_{i j}\right)=1$ ).

Using this definition of the local pressure and Eq. (3.1), we finally rewrite Eq. (3.8) as

$$
\begin{aligned}
\gamma_{\mathrm{SL}}+\gamma= & \int \mathrm{d} z\left[p_{\mathrm{n}}(z)-p_{\mathrm{t}}(z)\right] \\
& +\frac{1}{L_{x} L_{y}}\left\langle\sum_{s, i}\left[f_{z, i s}^{\mathrm{s}} z_{i s}-\frac{1}{2}\left(f_{x, i s}^{\mathrm{s}} x_{i}+f_{y, i s}^{\mathrm{s}} y_{i}\right)\right]-\sum_{s_{2}, i} f_{z, i s_{2}}^{\mathrm{s}} \Delta z\right\rangle,
\end{aligned}
$$

and the sum of the surface and interfacial tensions of the supported polymer film is given by the anisotropy of the pressure in the liquid and contributions due to the direct interaction between the liquid and the solid substrate. In the limit that the substrate is laterally homogeneous the terms involving the lateral forces between solid and liquid vanish. Since solid-liquid and liquid-vapor interfaces in a thick film are well separated by a bulk phase as shown in Fig. 3.3, the individual contributions for $\gamma_{\mathrm{SL}}$ and $\gamma$ can be set apart. It is done in the following section. 


\subsubsection{Solid-liquid and liquid-vapor interfacial tensions}

In the absence of a solid substrate, the liquid is separated by a liquid-vapor interface from its coexisting vapor phase. In this special case, Eq. (3.11) simplifies and allows us to measure the liquid-vapor interfacial tension through the anisotropy of the pressure tensor components across the interface as [45-47]:

$$
\gamma=\int_{z_{\text {top }}}^{z_{\mathrm{bot}}} \mathrm{d} z\left[p_{\mathrm{n}}(z)-p_{\mathrm{t}}(z)\right]
$$

We find $\gamma=0.512 \pm 0.006 \epsilon / \sigma^{2}$ which agrees well with previous calculations for similar systems [27]. Mechanical stability requires that the normal component of the pressure is constant throughout the system and equals the coexistence pressure [46]. Since the vapor pressure of a polymer melt is vanishingly small, $p_{\mathrm{n}}(z) \approx 0$. We also note, that the anisotropy of the pressure is localized around the interface and, therefore, the integration can be restricted to an interval $\left[z_{\text {bot }}, z_{\text {top }}\right]$ around the interface as shown in Fig. 3.3. At the temperature of $k_{\mathrm{B}} T / \epsilon=1.2$ the coexistence density of the liquid inside a thick polymer film is $\rho_{\text {coex }} \sigma^{3}=0.786$ (cf. Appendix A). The linear dimensions of all thick films in our simulations are $L_{x} \approx L_{y} \approx 50 \sigma$, whereas $L_{z}$ is chosen in a way to allow for the formation of the bulk region in the middle of the film.

If we consider a liquid in contact with the solid substrate, we can measure the solidliquid surface tension $\gamma_{\mathrm{SL}}$ according to Eq. (3.11) as

$$
\begin{aligned}
\gamma_{\mathrm{SL}}= & \int_{z_{\mathrm{bot}}^{\mathrm{SL}}}^{z_{\mathrm{top}}^{\mathrm{SL}}} \mathrm{d} z\left[p_{\mathrm{n}}(z)-p_{\mathrm{t}}(z)\right] \\
& +\frac{1}{L_{x} L_{y}}\left\langle\sum_{s, i}\left[f_{z, i s}^{\mathrm{s}} z_{i s}-\frac{1}{2}\left(f_{x, i s}^{\mathrm{s}} x_{i}+f_{y, i s}^{\mathrm{s}} y_{i}\right)\right]-\sum_{s_{2}, i} f_{z, i s_{2}}^{\mathrm{s}} \Delta z\right\rangle,
\end{aligned}
$$

where $z_{\text {bot }}^{\mathrm{SL}}$ and $z_{\mathrm{top}}^{\mathrm{SL}}$ stand for bottom and top limits of solid-liquid interface (cf. Fig. 3.3). Like in the case of the liquid-vapor interface, the anisotropy of the pressure, as well as the additional contribution due to the interaction between the liquid and the solid, are localized in a narrow region near the interface between the polymer liquid and the solid. The solid-liquid surface tension depends on the strength $\epsilon_{\mathrm{s}}$ of the attractive interaction between solid and polymer liquid. The simulation results are presented in Fig. 3.4.

If the droplet on a substrate depicted in Fig. 2.2 is at equilibrium, one may describe the equilibrium of forces acting on its contact line by the macroscopic Young equation that relates the interface energies and the equilibrium contact angle $\theta_{\mathrm{E}}[2,48]$,

$$
\gamma_{\mathrm{SL}}+\gamma \cos \theta_{\mathrm{E}}-\gamma_{\mathrm{SV}}=0
$$

Here, $\gamma_{\mathrm{SV}}$ is the solid-vapor interfacial tension. Since the vapor pressure is vanishingly small for our polymer melt, we can neglect the interfacial tension between the solid 


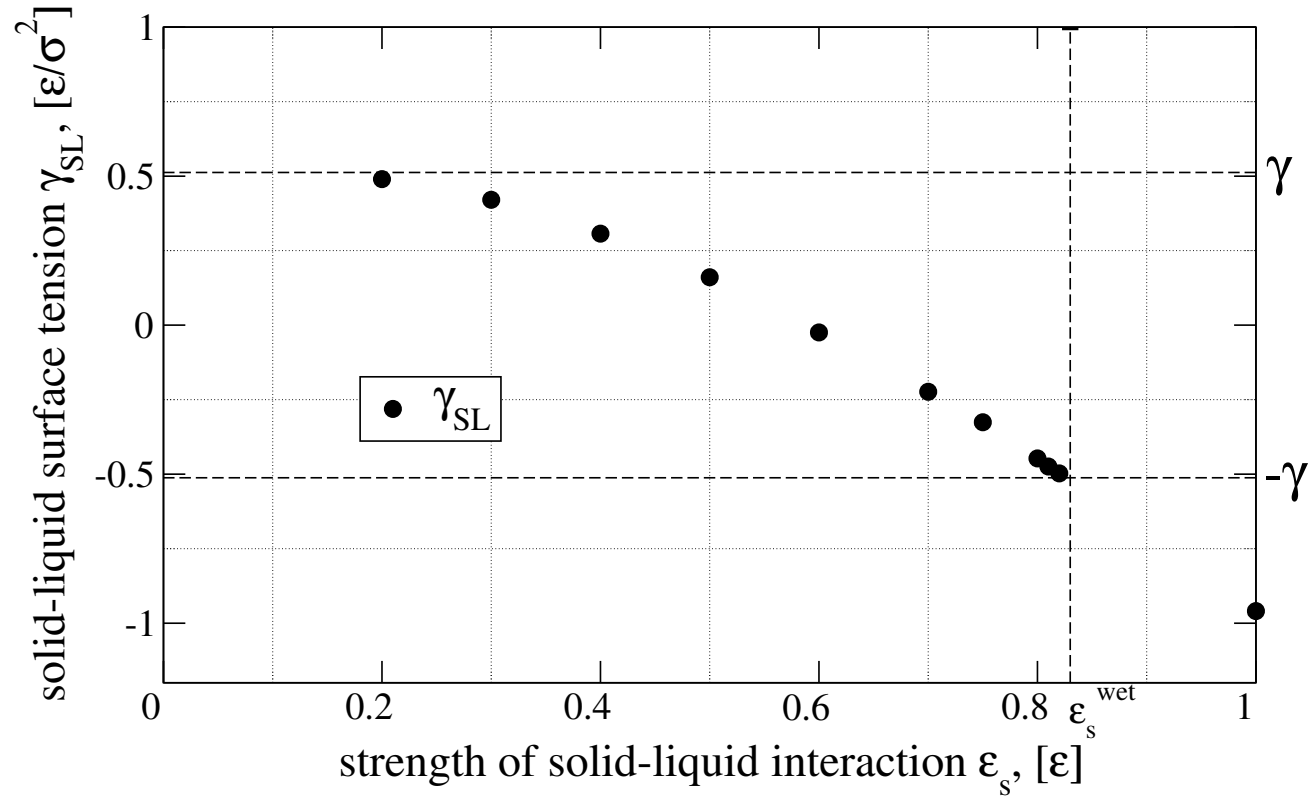

Figure 3.4: The dependence of the solid-liquid surface tension $\gamma_{\mathrm{SL}}$ on the strength of the solid-liquid interaction $\epsilon_{\mathrm{S}}$. The horizontal dashed lines represent the value of the liquidvapor interfacial tension $\gamma$ and value $-\gamma$. The vertical dashed line indicates the location of the wetting transition

substrate and the vapor phase, $\gamma_{\mathrm{SV}} \approx 0$ to a first approximation. Using this approximation, we find that the wetting and drying transitions occur at $\gamma_{\mathrm{SL}}\left(\epsilon_{\mathrm{S}}\right) \approx-\gamma$ and $\gamma_{\mathrm{SL}}\left(\epsilon_{\mathrm{S}}\right) \approx \gamma$, respectively. From the data in Fig. 3.4 we locate the wetting transition at $\epsilon_{\mathrm{s}} \approx 0.83 \epsilon$ and the contact angle reaches $180^{\circ}$ for small values of $\epsilon_{\mathrm{s}}<0.2 \epsilon$. A more rigorous approach to a determination of the wetting transition, although still based on approximation $\gamma_{S V} \approx 0$, is presented in the following section.

\subsubsection{Adhesion energy and wetting transition}

Separating the liquid from the substrate supporting it, two new interfaces, solid-vapor and liquid-vapor, are created. Thus, taking into account Young's relation (Eq. 3.14), the difference in free energy per unit area in the vicinity of the solid with respect to the initial situation can be written as [49]

$$
W=\left(\gamma_{\mathrm{SV}}+\gamma\right)-\gamma_{\mathrm{SL}}=\gamma+\gamma \cos \theta_{\mathrm{E}}=\gamma\left(1+\cos \theta_{\mathrm{E}}\right)
$$

The free energy $W$ is usually called adhesion energy. To describe the location of the wetting transition correctly, one can measure the adhesion energy while varying the strength $\epsilon_{\mathrm{S}}$ of the solid-liquid interaction. The wetting transition occurs at $\theta_{\mathrm{E}}=0$ that corresponds to $W_{0}=2 \gamma$.

An alternative method, based on the LJ solid-liquid interaction, can be employed to 
calculate the adhesion energy [27]. One invokes that it is simply the ratio of the free energy $F^{\mathrm{s}}$ to the area of solid-liquid interface $A_{\text {film }}=L_{x} L_{y}$. Taking into account that the partition function of a liquid in contact with a solid substrate is

$$
Z^{\mathrm{S}}=\frac{1}{N ! \lambda_{\mathrm{T}}^{3 N}} \int \prod_{i=1}^{N} d^{3} \vec{r}_{i} e^{-\beta \sum U_{\mathrm{LJ}}^{\mathrm{s}}\left(\epsilon_{\mathrm{s}}\right)-\beta \sum U_{\mathrm{LJ}},}
$$

and separating the $\mathrm{LJ}$ interaction amplitude $\epsilon_{\mathrm{s}}$ from the rest of the potential as $U_{\mathrm{LJ}}^{s}\left(\epsilon_{\mathrm{s}}\right)=$ $\epsilon_{\mathrm{s}} V_{\mathrm{s}}$, one can write the free energy as

$$
F^{\mathrm{S}}=-k_{\mathrm{B}} T \ln Z^{\mathrm{s}}=-k_{\mathrm{B}} T \ln \left(\frac{1}{N ! \lambda_{\mathrm{T}}^{3 N}} \int \prod_{i=1}^{N} d^{3} \vec{r}_{i} e^{-\beta \sum \epsilon_{\mathrm{s}} V_{\mathrm{s}}-\beta \sum U_{\mathrm{LJ}}}\right) .
$$

Differentiation with respect to $\epsilon_{\mathrm{s}}$ gives

$$
\begin{aligned}
\frac{\partial F^{\mathrm{s}}}{\partial \epsilon_{\mathrm{s}}}=-k_{\mathrm{B}} T \frac{1}{Z^{\mathrm{s}}} \frac{\partial Z^{\mathrm{s}}}{\partial \epsilon_{\mathrm{s}}}= & -k_{\mathrm{B}} T \frac{1}{Z^{\mathrm{s}}}\left(-\beta \sum V_{s}\right) \cdot Z^{\mathrm{s}} \\
= & <\sum V_{s}>=\frac{<\sum U_{\mathrm{LJ}}^{s}\left(\epsilon_{\mathrm{s}}\right)>}{\epsilon_{\mathrm{s}}},
\end{aligned}
$$

where angle brackets stand for ensemble averages. Now, differentiating Eq. (3.15) with respect to $\epsilon_{\mathrm{S}}$ under approximation of $\gamma_{\mathrm{SV}}=0$, one obtains

$$
\frac{\partial W}{\partial \epsilon_{\mathrm{s}}}=-\frac{\partial \gamma_{\mathrm{SL}}}{\partial \epsilon_{\mathrm{s}}}=-\frac{1}{A} \frac{\partial F^{\mathrm{s}}}{\partial \epsilon_{\mathrm{s}}}
$$

where we have also taken into account that $F^{\mathrm{s}}=A \gamma_{\mathrm{SL}}$. The adhesion energy then takes the form

$$
W=-\frac{1}{A_{\text {film }}}\left(\int_{0}^{\epsilon^{\prime}} d \epsilon_{\mathrm{s}} \frac{<\sum U_{\mathrm{LJ}}^{s}\left(\epsilon_{\mathrm{s}}\right)>}{\epsilon_{\mathrm{s}}}+C\right),
$$

where $C$ is an unknown constant of integration.

The results of both methods are plotted in Fig. 3.5. The thick solid horizontal line marks the adhesion energy at the wetting transition, $W_{0}=2 \gamma$. The circles represent the values obtained from surface and interfacial tensions, while the dashed line stands for the integration method. We find a good agreement between both methods and the wetting transition can be localized at $\epsilon_{\mathrm{s}}^{\text {wet }} \approx 0.83 \epsilon$. This result is obtained under assumption $\gamma_{\mathrm{SV}} \approx 0$. This approximation is very good at small values of the solid-liquid interaction strengths $\epsilon_{\mathrm{s}}$, but close to the wetting transition it is no longer the case. The next section is devoted to the estimation of the solid-vapor interfacial tension in the regime when assumption $\gamma_{\mathrm{SV}} \approx 0$ is not reliable anymore.

\subsubsection{Solid-vapor interfacial tension}

While the approximation $\gamma_{\mathrm{SV}} \approx 0$ is appropriate for small values of the strength of attractive solid-liquid interactions, $\epsilon_{\mathrm{s}}$, the quality of this approximation deteriorates in 


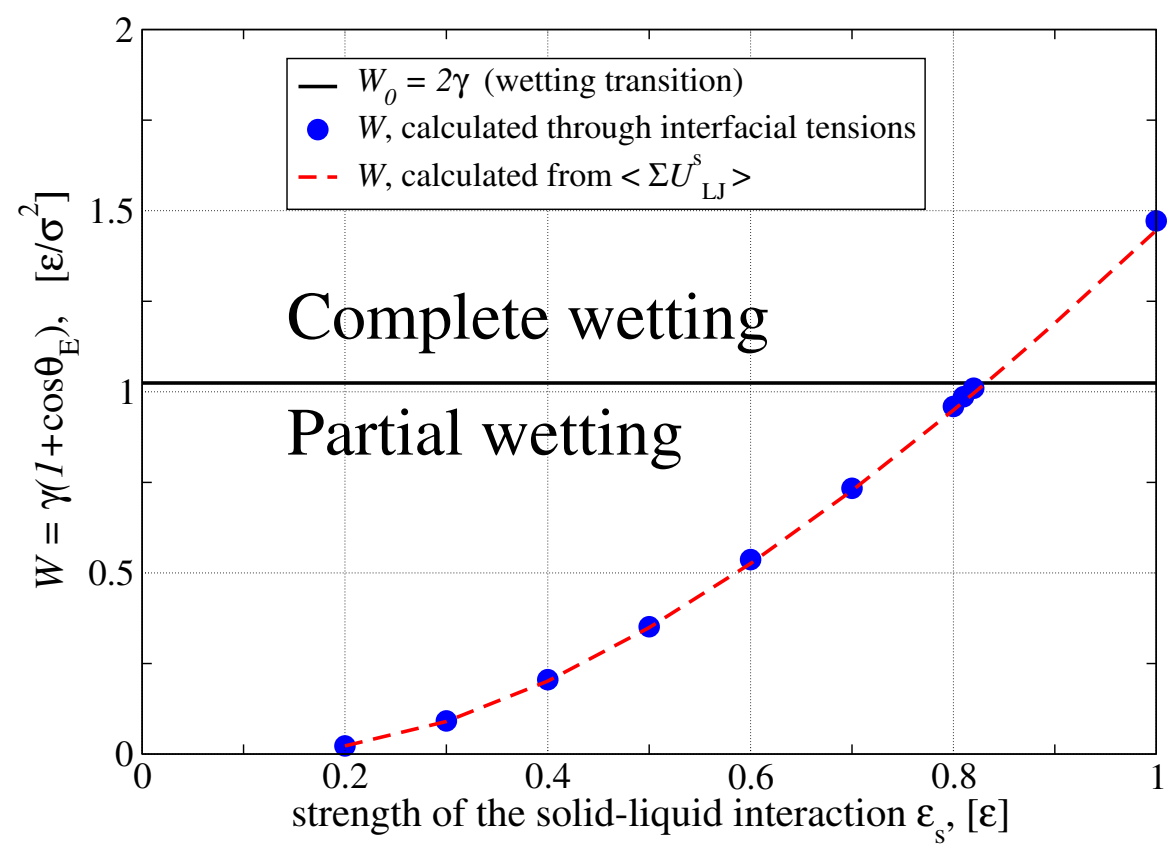

Figure 3.5: Adhesion energy per unit area defined by two methods: direct calculation from surface and interfacial tensions (circles) and integration method (dashed line). The thick solid horizontal line marks the adhesion energy $W_{0}=2 \gamma$. The wetting transition occurs at $\epsilon_{\mathrm{s}}^{\text {wet }} \approx 0.83 \epsilon$.

the vicinity of the wetting transition. If the wetting transition were of second-order, the amount of liquid adsorbed onto the substrate, would continuously diverge as we approach the wetting transition. Even for a first-order wetting transition we expect that the adsorbed amount will increase when $\epsilon_{\mathrm{s}}$ increases towards its transition value. In this case the approximation $\gamma_{\mathrm{SV}} \approx 0$ becomes unreliable, and we employ a meniscus geometry as shown in Fig. 3.6 to extract the value of the solid-vapor tension. The film thickness is chosen sufficiently large, such that the deviation of the pressure from its coexistence value, $\Delta p \sim-\gamma\left(\frac{1}{R_{x}}+\frac{1}{R_{y}}\right)$, with $R_{x}$ and $R_{y}=\infty$ denoting the principle radii of curvature of the meniscus, has only a small influence on the adsorbed amount of polymer and $\gamma_{\mathrm{SV}}{ }^{1}$. Since $\Delta p<0$, the adsorbed amount in the simulations will be smaller than at coexistence, $\gamma_{S V}$ will be too large (i.e., negative $\gamma_{S V}$ will have an absolute value that is too small), and we will slightly underestimate the contact angle, $\theta_{\mathrm{E}}$. This correction to the deviation of the approximation $\gamma_{\mathrm{SV}} \approx 0$, however, is insignificant for

${ }^{1}$ One can estimate the error due to the finite radius of the curvature $R_{x}$ of the meniscus as follows:

(i) from Gibbs adsorption isotherm, $\frac{\partial \gamma_{\mathrm{SV}}}{\gamma \mu}$, where $\Gamma_{\mathrm{SV}}$ is the number of vapor molecules per unit area of the substrate and (ii) the vapor is so dilute, that it can be described by an ideal gas of chains. Its equation of state is $p=\frac{N}{V} k_{\mathrm{B}} T$ and the chemical potential is $\mu=k_{\mathrm{B}} T \ln \frac{N \lambda_{\mathrm{T}}^{3}}{V f}$, where $f$ is the degeneracy of states. Then, $\mu=k_{\mathrm{B}} T \ln \frac{p \lambda_{\mathrm{T}}^{3}}{k_{\mathrm{B}} T f}$ and $\left.\frac{\partial \mu}{\partial p}\right|_{T}=\frac{k_{\mathrm{B}} T}{p}$.

Then, the error in solid-vapor interfacial tension is $\Delta \gamma_{\mathrm{SV}}=-\int \mathrm{d} \mu \Gamma_{\mathrm{SV}}=-\left.\int \mathrm{d} p \frac{\partial \mu}{\partial p}\right|_{T} \Gamma_{\mathrm{SV}}=$ $-\int \mathrm{d} p \frac{k_{\mathrm{B}} T}{p} \Gamma_{\mathrm{SV}}=-k_{\mathrm{B}} T \int \mathrm{d}(\ln p) \Gamma_{\mathrm{SV}}(p)$. Taking into account that $\Gamma_{\mathrm{SV}}(p)=c p$, we can rewrite the error by $\Delta \gamma_{\mathrm{SV}}=-k_{\mathrm{B}} T c \Delta p=-k_{\mathrm{B}} T\left[\Gamma_{\mathrm{SV}}\left(p_{\text {coex }}\right)-\Gamma_{\mathrm{SV}}\left(p_{\text {coex }}-\frac{\gamma}{R_{x}}\right)\right]$. 


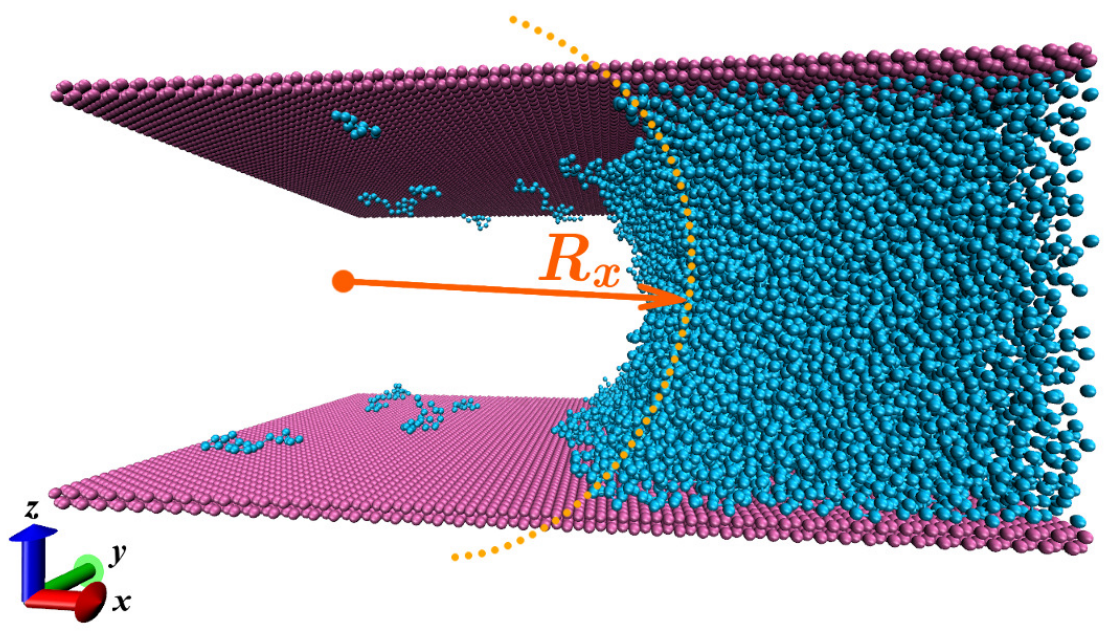

Figure 3.6: A part of a system used to determine solid-vapor interfacial tension $\gamma_{\mathrm{SV}}$. The droplet serves as a reservoir to the chains adsorbed on the substrate. The yellow dotted line indicates the curvature of the liquid-vapor interface. The radius of curvature $R_{x}$ is indicated by the orange arrow.

\begin{tabular}{l|c|c|c|c} 
& $\epsilon_{\mathrm{S}}=0.75 \epsilon$ & $\epsilon_{\mathrm{S}}=0.80 \epsilon$ & $\epsilon_{\mathrm{s}}=0.81 \epsilon$ & $\epsilon_{\mathrm{S}}=0.82 \epsilon$ \\
\hline$\gamma_{\mathrm{SV}},\left[\epsilon / \sigma^{2}\right]$ & 0 & -0.00281 & -0.00475 & $-0.00523(-0.01642)$ \\
\hline$\gamma_{\mathrm{SL}},\left[\epsilon / \sigma^{2}\right]$ & -0.32576 & -0.44737 & -0.47419 & -0.49761 \\
\hline$\theta_{\mathrm{E}_{0}}\left(\right.$ at $\left.\gamma_{\mathrm{SV}}=0\right)$, [degree] & 50.50 & 29.14 & 22.20 & 13.69 \\
\hline$\theta_{\mathrm{E}},[$ degree] & 50.50 & 29.77 & 23.57 & $15.98(20.03)$
\end{tabular}

Table 3.1: Interfacial tensions of solid-vapor and solid-liquid interfaces and contact angles with $\left(\theta_{\mathrm{E}}\right)$ and without $\left(\theta_{\mathrm{E}_{0}}\right)$ taking the solid-vapor interfacial tension into account. For $\epsilon_{\mathrm{S}}=0.82 \epsilon$ the value $\gamma_{\mathrm{SV}}$ is affected by the finite value of $\Delta p$ and we provide in parentheses an alternative estimate of the contact angle.

the used system size for all values of $\epsilon_{\mathrm{s}}$ but the close vicinity of the wetting transition (at $\epsilon_{\mathrm{s}} \approx 0.83 \epsilon$ ), where we have used an alternative methods as described in Sec. 3.1.5.

For the calculation of $\gamma_{\mathrm{SV}}$ we used the same procedure as earlier for the solid-liquid surface tensions of a film, but the procedure is only applied to the part of the simulation box that is far away from the meniscus-forming liquid bridge. The dimensions of the simulation domain were chosen in a way to provide a reasonably big principle radius $R_{x}$, namely $L_{x}=150 \sigma, L_{y}=30 \sigma$ and $L_{z}=50 \sigma$. In the vicinity of the wetting transition, $R_{x} \approx L_{z} / 2$. The values of $\gamma_{\mathrm{SV}}$ and $\gamma_{\mathrm{SL}}$ (for comparison) are presented in Table (3.1). One notices the increase in $\gamma_{S V}$ when the wetting transition is approached. However, compared to the influence on the solid-liquid surface tension the effect is small. Nevertheless, it becomes the more important the closer one comes to the wetting transition, and the correction of the contact angles is significant when one compares profiles of drops of different sizes with the prediction of Eq. (3.14). 


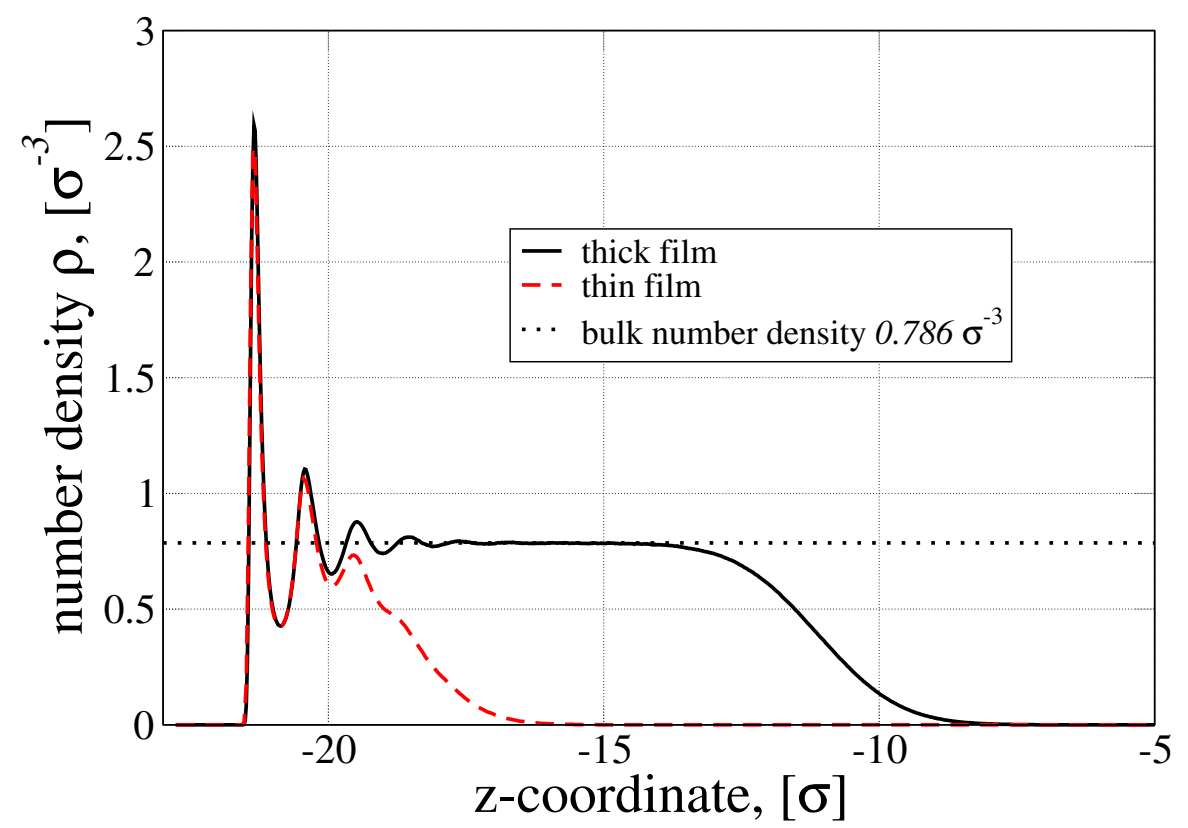

Figure 3.7: Density profiles of a polymer film at $\epsilon_{\mathrm{s}}=0.80 \epsilon$. The solid black line represents a thick film with a bulk region separating solid-liquid and liquid-vapor interfaces. In the case of a thin film (dashed red line) it is no longer possible to distinguish the two interfaces. The dotted horizontal line indicates the coexistence number density.

Now, as we identified the wetting transition and estimated the values of the solid-vapor interfacial tension $\gamma_{\mathrm{SV}}$ as a function of the solid-liquid interaction strength, $\epsilon_{\mathrm{S}}$, a stability analysis of liquid films in the vicinity of the wetting transition can be performed. The following section introduces the concept of interface potential and provides results on the stability of the films of different thicknesses.

\subsubsection{Interface potential and Derjaguin pressure}

If we consider a thin polymer film on top of the solid substrate, Eq. (3.1) should be generalized accounting not only for the solid-liquid and liquid-vapor interfacial tensions, $\gamma_{\mathrm{SL}}$ and $\gamma$, but also for the interface potential, $g(h)$, that quantifies the free-energy cost of locating the liquid-vapor interface a distance $h$ away from the solid substrate [39]:

$$
\begin{aligned}
\left.\frac{\mathrm{d} F(\Lambda)}{\mathrm{d} \Lambda}\right|_{\Lambda=1} & =\left.\left[\gamma_{\mathrm{SL}}+\gamma+g(h)\right] \frac{\mathrm{d} L_{x}^{\prime} L_{y}^{\prime}}{\mathrm{d} \Lambda}\right|_{\Lambda=1}+\left.\frac{\mathrm{d} g(h)}{\mathrm{d} h} \frac{\mathrm{d} h^{\prime}}{\mathrm{d} \Lambda}\right|_{\Lambda=1} L_{x} L_{y} \\
& =\left[\gamma_{\mathrm{SL}}+\gamma+g(h)-\frac{\mathrm{d} g(h)}{\mathrm{d} h} h\right] L_{x} L_{y},
\end{aligned}
$$

For a thick film (cf. Fig. 3.7), the transitions in the polymer density at the two interfaces are well separated, and the density at the center of the film approaches the bulk coexistence value, as was shown above in Sec. 3.1.2. In this case, also the contributions to Eq. (3.11) that stem from the two interfaces can be well separated. The anisotropy of 
the pressure tensor at the solid substrate gives $\gamma_{\mathrm{SL}}$, and the one at the liquid-vapor interface yields $\gamma$. Thus, the interface potential vanishes, $g(h \rightarrow \infty) \rightarrow 0$, indicating that the liquid-vapor interface will not interact with the substrate if the film is sufficiently thick. The free energy of the film is then $F(h \rightarrow \infty) \rightarrow F_{0}$ (cf. Eq. 3.1).

However, upon decreasing the film thickness, the two interfaces start to interact and the contributions of the solid-liquid and liquid-vapor interfaces can not be separated anymore. The interaction between the interfaces is quantified by the interface potential, $g(h)$, or equivalently, by the Derjaguin pressure $\Pi(h)=-\frac{\mathrm{d} g(h)}{\mathrm{d} h}$. From Fig. 3.7 we observe that for small film thickness both interface density profiles are distorted, and the density does not reach its coexistence value at the center of the film. The distortion of the density profile far away from the interfaces is characterized by the bulk correlation length, $\xi_{0}$, which therefore sets the length scale of the interface potential [50].

Several strategies have been proposed to measure the interface potential in computer simulation of particle-based models: (i) The interaction between the interface and the substrate can be obtained in the grandcanonical ensemble, where the chemical potential $\mu$ controls the fluctuating thickness of the wetting layer of the liquid on the substrate. The probability, $P(h)$, of observing a wetting layer of thickness $h$ is related to the interface potential via $g(h)=-k_{\mathrm{B}} T \ln P(h)+$ const [51-54], where the choice of the constant ensures the boundary condition $g(h \rightarrow \infty)=0$. While being elegant, this computational technique is limited to simple models because the grandcanonical ensemble requires the insertion and deletion of polymers and concomitant Monte-Carlo moves are only efficient for short polymers, low densities or in the vicinity of the liquid-vapor critical point. (ii) A negative curvature of the interface potential at a thickness $h$ signals the spontaneous instability of a wetting layer. From the characteristic length scale of this spinodal dewetting pattern one can deduce information about $d^{2} g(h) / d h^{2}[55,56]$. (iii) Here we use the pressure tensor. This is a general technique that is not limited to short polymers or low densities. It does not require the implementation of particle insertion/deletion Monte-Carlo moves and can be straightforwardly implemented in standard Molecular Dynamics program packages.

Using the definitions of the local pressures Eqs. (3.9) and (3.10) and Eq. (3.21), we rewrite Eq. (3.8) for thin films as [39]

$$
\begin{aligned}
\gamma_{\text {film }}(h) \equiv & \gamma_{\mathrm{SL}}+\gamma+g(h)-\frac{\mathrm{d} g(h)}{\mathrm{d} h} h=\int \mathrm{d} z\left[p_{\mathrm{n}}(z)-p_{\mathrm{t}}(z)\right] \\
& +\frac{1}{L_{x} L_{y}}\left\langle\sum_{s, i}\left[f_{z, i s}^{\mathrm{s}} z_{i s}-\frac{1}{2}\left(f_{x, i s}^{\mathrm{s}} x_{i}+f_{y, i s}^{\mathrm{s}} y_{i}\right)\right]-\sum_{s_{2}, i} f_{z, i s_{2}}^{\mathrm{s}} \Delta z\right\rangle
\end{aligned}
$$

We particularly stress that in the canonical ensemble the difference of the film tension $\gamma_{\text {film }}(h)$ and interfacial tensions $\gamma_{\mathrm{SL}}$ and $\gamma$ is not the interface potential $g(h)[40,41,57]$, but of the form of Legendre transform $g(h)-h \frac{\mathrm{d} g(h)}{\mathrm{d} h}$. 
Since we have determined $\gamma_{\mathrm{SL}}$ and $\gamma$ independently, we are able to extract the interface potential, $g(h)$, from the simulation data for thin films. To this end, we have to define the location of the liquid-vapor interface, i.e., the film thickness, $h$. There are several options: Either (i) one determines the position where the density equals a predefined value, typically $\left(\rho_{\text {liq }}+\rho_{\text {vap }}\right) / 2$ (crossing criterion) or (ii) one defines the film thickness via the adsorbed excess (Gibbs dividing surface),

$$
\Delta \Gamma_{\text {ads }}=L_{x} L_{y} \int \mathrm{d} z\left[\rho(z)-\rho_{\text {vap }}\right] \equiv\left[\rho_{\text {liq }}-\rho_{\text {vap }}\right] L_{x} L_{y} h
$$

In this work we adopt the integral criterion (3.23) to define the film thickness. Neglecting the vanishingly small vapor density at coexistence, we obtain

$$
h_{\text {eff }}=\frac{N_{\text {mon }}}{\rho_{\text {liq }} A_{\text {film }}},
$$

where $N_{\text {mon }}$ is the number of monomers of the liquid inside the simulation box and $A_{\text {film }}=L_{x} L_{y}$ is the area of the substrate underneath the film.

We note that both definitions become problematic for film thicknesses where the curvature of the interface potential is negative, $\frac{\mathrm{d}^{2} g}{\mathrm{~d} h^{2}}<0$. In this regime of film thicknesses a laterally extended, homogeneous film becomes unstable with respect to spinodal dewetting $[55,58,59])$. However, even in this film thickness region, the films can be linearly or even absolutely stable if the lateral extension of the simulation box is sufficiently small. The related critical values depend on film thickness (see, e.g., Fig. 8 of [60]). In the simulation, we can still obtain meaningful data for the interface potential if we restrict the lateral system size to be smaller than the characteristic wavelength of the spontaneous rupture process. For simulations of thin films we reduce lateral sizes of the system to $L_{x} \approx L_{y} \approx 15 \sigma$.

Additionally, we mention that the liquid-vapor interface in our Molecular Dynamics simulations exhibits local fluctuation of its height (i.e., capillary waves), and the Gibbs dividing surface measures the laterally averaged film thickness. The interaction of the liquid-vapor interface with the substrate imparts a lateral correlation length, $\xi_{\|}=2 \pi \sqrt{\gamma / \frac{\mathrm{d}^{2} g}{\mathrm{~d} h^{2}}}$, onto these interface fluctuations. These fluctuations give rise to a weak dependence of the interface potential on the lateral system size for $L_{x}, L_{y}<\xi_{\|}$, i.e., the interface potential is renormalized by interface fluctuations. Qualitatively, the effect of fluctuations is to extend the range of the potential, i.e., $\xi=\xi_{0}(1+\omega / 2)$ with $\omega=\frac{k_{B} T}{4 \pi \tilde{\xi}_{0}^{2} \gamma}[61]$

The interface potential exhibits a minimum at small film thickness, $h_{\min }$. This film thickness characterizes the amount of liquid adsorbed on the substrate in contact with the vapor. As illustrated in Sec. 3.1.4, $h_{\min }=0$ except for the close vicinity of the wetting transition. The free energy of such a vanishingly thin polymer film is given by 
$\gamma_{\text {film }}\left(h_{\text {min }}\right)=\gamma_{\mathrm{SL}}+\gamma+g\left(h_{\min }\right)=\gamma_{\mathrm{SV}}$. Thus, the measurement of the different tensions for a planar polymer film provides the value of $g\left(h_{\min }\right)$.

Alternatively, we can use the measured value $g\left(h_{\min }\right)$, in turn, to estimate the solidvapor tension, $\gamma_{\mathrm{SV}}$. We have employed this strategy for $\epsilon_{\mathrm{S}}=0.82 \epsilon$, where the finite curvature of the meniscus resulted in a relevant deviation of the pressure from its coexistence value. Extrapolating the simulation data to the thickness $h_{\min } \approx 0$ we obtain $\gamma_{\mathrm{SV}}=-0.01642$. We will use this more accurate value, which is not affected by the curvature of the meniscus and that is compatible with the interface potential, in the comparison with the CD in Sec. 3.2.

Since Eq. (3.22) only provides the Legendre transformation of the interface potential and we require an analytical expression for bridging later the particle-based MD simulations to a CD in Secs. 3.2.2 and 3.2.3, we make an Ansatz for the functional form of $g(h)$. Generally, one can distinguish between short-range and long-range contributions to the interface potential [50,62]. The long-range contribution results from dispersion forces between the liquid and the substrate. In our particle-based model, however, we do only consider the short-range part as our LJ interaction (2.2) is cut off at $r_{c}$. Thus, there is no long-range contribution in our model in contrast to previous works, when an effective long-range contribution was taken into account to calculate Hamaker constant despite finite interaction cut off $[40,41]$. The short-range contribution to $g(h)$ stems from the distortion of the interface profile due to the nearby presence of the solid substrate as illustrated in Fig. 3.7, and it is typically expanded in a series of exponentials $[50,62]$

$$
g_{\mathrm{sr}}(h)=a e^{-h / \xi}-b e^{-2 h / \xi}+c e^{-3 h / \xi}-d e^{-4 h / \xi}+\ldots,
$$

In order to obtain $g(h)$ in practice, we fit its Legendre transform $g(h)-h \frac{\mathrm{d} g}{\mathrm{~d} h}$ by a sum of four exponential terms like in Eq. (3.25), and enforce that the interface potential exhibits a minimum at $h_{\min } \approx 0$ (i.e. there is no precursor film in our MD model) with a value $g\left(h_{\min }\right)$, as obtained by the measurement of the interfacial tensions. The resulting fits for $g(h)$ at $\epsilon_{\mathrm{s}}=0.75 \epsilon, 0.80 \epsilon, 0.81 \epsilon$ and $0.82 \epsilon$ are given as solid lines in Figs. 3.8a-3.8d. The parameters of the fits are presented in Table (3.2).

Using the macroscopic Young-Dupré relation, one observes that value of the minimum of $g(h)$ dictates the contact angle [39]

$$
g\left(h_{\min }\right)=\gamma\left(\cos \theta_{\mathrm{E}}-1\right)
$$

Much more information can be extracted from the interface potential: (i) the stability regions of films of various thicknesses may be defined as shown in Fig. 3.9. Films with thicknesses such that $g^{\prime \prime}(h)<0$ are linearly unstable and tend to dewet spinodally. In other cases, the films either stable or metastable [10]. (ii) the minimum of the interface 


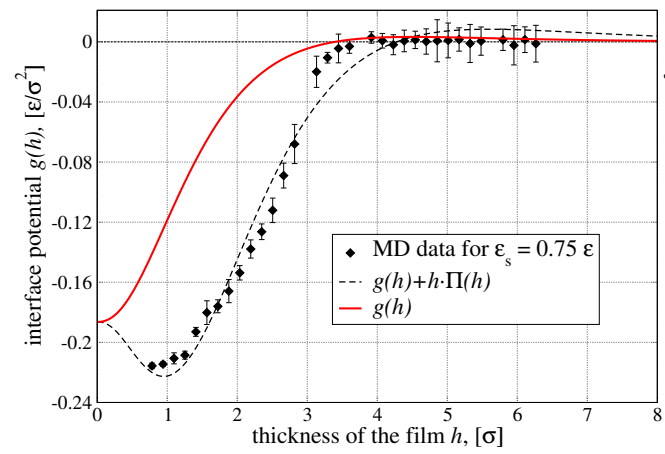

(a)

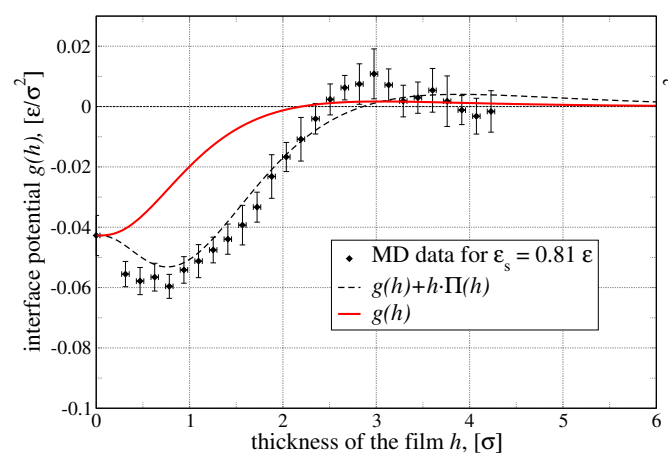

(c)

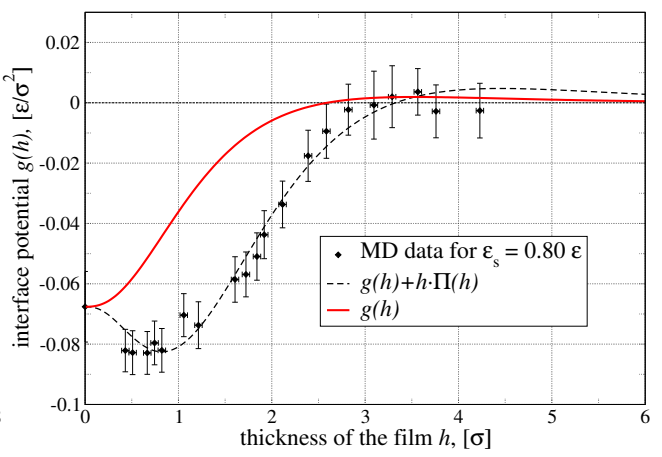

(b)

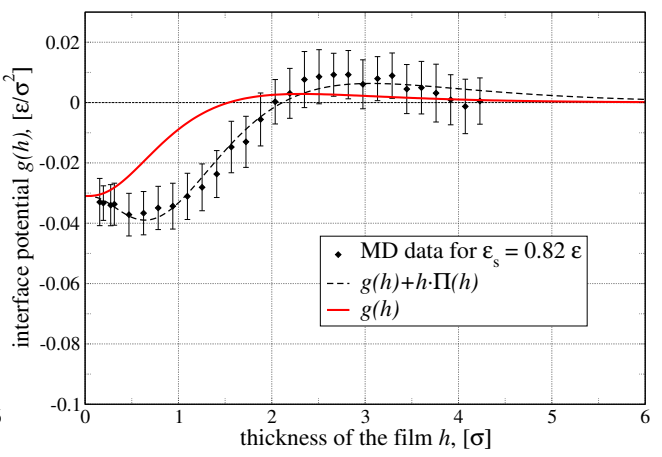

(d)

Figure 3.8: Panels (a), (b), (c) and (d) give the interface potential $g(h)$ at $\epsilon_{\mathrm{s}}=0.75 \epsilon$, $\epsilon_{\mathrm{s}}=0.80 \epsilon, \epsilon_{\mathrm{s}}=0.81 \epsilon$ and $\epsilon_{\mathrm{s}}=0.82 \epsilon$, respectively. They are obtained by fitting the MD results for the tension $\gamma_{\text {film }}(h)-\gamma-\gamma_{\mathrm{SL}}$ of films of various small thicknesses (black symbols with error bars) by the expression $g(h)+h \Pi(h)$ (dashed black line) obtained employing the first four terms of the short-range part of the interface potential $g_{\mathrm{sr}}(h)$. The resulting interface potential $g(h)$ is given as solid red line. Note, that the minimal value of $g_{\min }$ is always reached at vanishingly small thicknesses $h \approx 0 \sigma$, as there is no precursor film in our particle-based model.

potential defines the macroscopic contact angle according to Eq. 3.26. (iii) The observation that $g(h)$ increases above zero at intermediate values of $h$ indicates that the wetting transition is of the first order.

The interface potential can give additional information if we do no longer consider only film geometries, but turn to droplets. Independently of the apex height of the drop, the interface potential (or Derjaguin pressure) plays an important role in the vicinity of the three-phase contact line, as a steady change of the position of liquid-vapor interface is taking place. The interface potential provide the following information that is relevant in case of droplets: (i) The shape of the interface potential controls deviations of the drop shape from a spherical cap in the vicinity of the wetting transition. (ii) Within the square-gradient approximation the integral of $\sqrt{g(h)}$ is related to the line tension at the three-phase contact line [63]. For all values of $\epsilon_{\mathrm{s}}$ investigated in the particle-based 


\begin{tabular}{l|c|c|c|c} 
Parameter & $\epsilon_{\mathrm{S}}=0.75 \epsilon$ & $\epsilon_{\mathrm{S}}=0.80 \epsilon$ & $\epsilon_{\mathrm{S}}=0.81 \epsilon$ & $\epsilon_{\mathrm{S}}=0.82 \epsilon$ \\
\hline$a,\left[\epsilon / \sigma^{2}\right]$ & 0.13191 & 0.06485 & 0.05057 & 0.06132 \\
\hline$b,\left[\epsilon / \sigma^{2}\right]$ & 1.40871 & 0.58700 & 0.41256 & 0.36875 \\
\hline$c,\left[\epsilon / \sigma^{2}\right]$ & 1.67606 & 0.70902 & 0.50249 & 0.42963 \\
\hline$d,\left[\epsilon / \sigma^{2}\right]$ & 0.58566 & 0.25447 & 0.18323 & 0.15318 \\
\hline$\xi,[\sigma]$ & 1.51770 & 1.26964 & 1.14735 & 1.00512
\end{tabular}

Table 3.2: Parameters of the fitting curves of $g(h)$ for the case where the first four terms of the short-range contributions [Eq. (3.25)] are taken into account. Note, that only three parameters are independent since there are two additional constraints: The local minimum criterion at $h \approx 0 \sigma$ implies $d=(a-2 b+c) / 4$ and the Young-Dupré relation 3.26 dictates the value $g_{\min }$ by setting $b=a+c-d-g_{\min }=1.5 a+0.5 c-2 g_{\min }$.

model, the line tension is expected to be negative.

In the next section, we focus on droplets in the vicinity of the wetting transition. A technique passing relevant parameters from particle-based simulations to a CD is suggested and tested by comparison of droplet profiles obtained by both methods.

\subsection{Static properties of polymer liquids at partial wetting}

In the previous decade increasing attention has focused on the behavior of small quantities of liquid on hard $[10,59,64-68]$ or soft $[69,70]$ substrates in equilibrium or under the influence of driving forces parallel to the substrate [27, 71, 72]. The current section mainly considers two levels of description: particle-based models [28, 34, 52, 67, $73-$ 76] and continuum theory [10, 65, 68, 71, 72, 77-79]. The former describes the liquid in terms of the position and momenta of particles. The properties of particle- based models are studied by discrete stochastic simulations, i.e., Monte-Carlo simulation or Molecular Dynamics. The advantage of retaining the particle degrees of freedom consists of the ability to refine the model towards a chemically realistic description and to include effects of thermal fluctuations and of discreteness of matter that are expected to become important on small length scales. However, these stochastic simulation techniques are limited to droplets of a linear size that does not exceed a few nanometers.

Continuum models, in turn, describe the liquid in terms of collective variables that do not refer to individual particles. Typical examples of continuum theories are the hydrodynamic description in terms of the density and momentum fields or interface models that describe the liquid only through a characterization of the motion of its liquid-vapor boundary. Continuum descriptions can address engineering time and length scales but depend on phenomenological material constants that are often not related in a straightforward way to the microscopic interactions of the particle-based description. Thus effort has to be devoted to parameter-passing techniques that transfer information from 


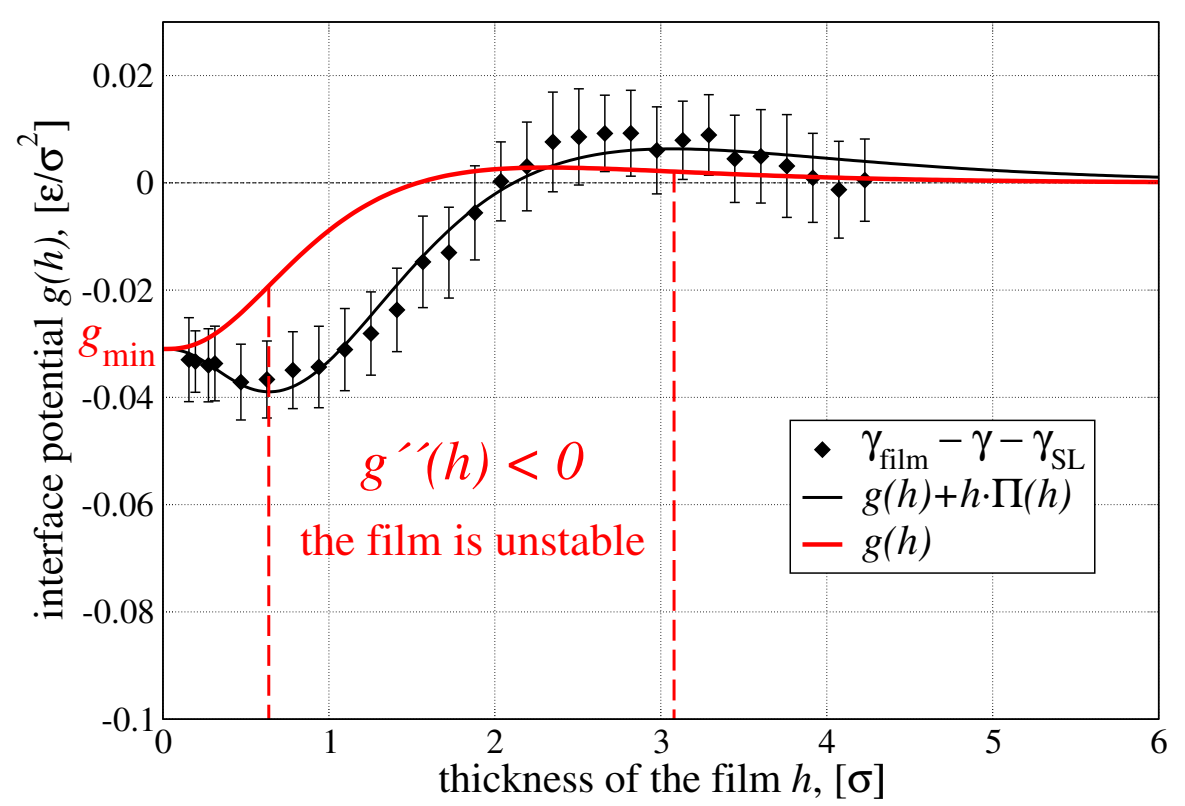

Figure 3.9: The Legendre transform (black dots fitted by an analytical representation) and interface potential of the films of various thicknesses at $\epsilon_{\mathrm{s}}=0.82 \epsilon$. The region of unstable film thicknesses, where $g^{\prime \prime}(h)<0$, is marked by vertical dashed lines. The position of the minimum, $g_{\mathrm{min}}$, dictates the macroscopic contact angle.

particle-based models to the continuum description. To this end, two questions have to be addressed: (i) Which is the relevant information of the particle-based model needed in the continuum description and (ii) how can one extract this information from the particle-based description in the appropriate continuum form?

In the present section, in addition to the coarse-grained particle model of a polymer drop on a solid substrate, we use a thin film description that characterizes the droplet shape by the location, $h$, of the liquid-vapor interface above the substrate. We explore the behavior of small nanodrops where both descriptions are computationally feasible. We extract the interfacial tensions and the Derjaguin or disjoining pressure [80, 81] from Molecular Dynamics simulation of the particle-based model and pass them to continuum model. Then both approaches are used to determine the equilibrium contact angle of a droplet as a function of the size of the droplet and of interaction strength between the liquid and the substrate.

To our knowledge, such a parameter passing scheme has not yet been developed for the case of liquid droplets on solid substrates. However, the disjoining pressure itself can be extracted in grandcanonical ensemble [51-54, 82]. Additionally, related works exist for other geometries in canonical ensemble, such as free standing films or films adsorbed in pores [57].

Bhatt et al. [40] extract a disjoining pressure as a function of chemical potential from MD simulations for a free standing film of a volatile Lenard-Jones liquid and compare 
the results with the ones of density functional theory. Their approach consists in the definition of the disjoining pressure as the difference of normal pressure in the film and the pressure in the homogeneous liquid at the same chemical potential as the film. However, as discussed in section 3.1.5, the measurement of the chemical potential in a canonical ensemble is difficult and requires additional simulations. Moreover, despite of truncated potentials, they relate the disjoining pressure with solely long-range van der Waals dispersion forces and provide therefore comparison to Hamaker theory. The short-range forces stay outside the scope of their research.

A planar liquid film bounded by a solid and vapor is studied by Han [41] using grandcanonical MD simulations with a truncated and shifted Lennard-Jones interaction. The disjoining pressure is extracted in a similar way as in Ref. [40] and again is associated with only long-range dispersion forces.

Note that parameter passing from MD simulations to continuum hydrodynamics is also frequently done in the context of liquid flow close to solid substrates [66, 83-87]. However, since these works do either not involve free interfaces [85, 87] or do not extract the disjoining pressure $[66,84,86]$, we do not discuss them here further.

This section is structured as follows. First, in section 3.2.1 we formulate a continuum approach to describe droplets on solid surfaces and determine parameters that have to be passed into $C D$ from the particle-based simulations. Then, section 3.1.5 details how we calculate the passing parameters in particle-based MD simulations. The subsequent section 3.2.2 presents the dependence of the equilibrium contact angle on droplet size for various interaction energies between the liquid and the substrate. In passing, we describe several ways to define the equilibrium contact angle and discuss their relation to the macroscopic Young-Laplace law. Finally, section 3.2.3 provides a comparison of droplet profiles as obtained by particle-based and continuum models.

Current work is a joint project with Desislava Todorova and Uwe Thiele (Loughborough University, UK), who performed the numerical simulations of the continuum model. The results are summarized in the article submitted to J. Chem. Phys.

\subsubsection{Drops as described by continuum description (CD)}

We employ a highly coarse-grained description to characterize the free-energy of a droplet on a planar substrate in terms of the position of the solid-liquid and liquidvapor interfaces. Generally, the free energy takes the translationally and rotationally invariant form

$$
F=\gamma_{\mathrm{SL}} \int_{\mathrm{SL}} \mathrm{d} S+\gamma \int_{\mathrm{LV}} \mathrm{d} S+\int_{\mathrm{LV}} \mathrm{d} S \int_{\mathrm{SL}} \mathrm{d} S^{\prime} \tilde{g}\left(\left|\mathbf{r}-\mathbf{r}^{\prime}\right|\right)
$$


where the integrals extend over the solid-liquid (SL) and liquid-vapor (LV) interfaces ${ }^{2}$. In Eq. (3.27), $\gamma_{S \mathrm{~S}}$ is the free energy per unit contact area of the liquid and the solid substrate, and $\gamma$ denotes the liquid-vapor interfacial tension. The last term of Eq. (3.27) describes the effective interaction between the interfaces, and $\mathbf{r}$ and $\mathbf{r}^{\prime}$ are points on the liquid-vapor and solid-liquid interface, respectively. In the following, we restrict our attention to $2 \mathrm{~d}$ droplets on a planar substrate (cf. Fig. 2.2), choose the $x$-coordinate along the planar solid substrate and denote by $z=h(x)$ the local distance between a point $\mathbf{r} \equiv(x, y, z=h(x))$ of the liquid-vapor interface and the planar substrate (Monge representation). Due to cylindrical geometry, one may (i) study drops of bigger radii than in the case of spherical droplets (at the same number of monomers) [27] and (ii) neglect line tension effects, as three-phase contact lines are on average straight [88] and their length is independent of the droplet size and shape.

The interaction of a point on the (planar) liquid-vapor interface (parallel to the substrate) with the solid is obtained by integrating over the substrate area

$$
g(h)=\int_{\mathrm{SL}} \mathrm{d} S^{\prime} \tilde{g}\left(\left|\mathbf{r}-\mathbf{r}^{\prime}\right|\right),
$$

which for a homogeneous substrate only depends on the distance, $h$, due to symmetry. $g(h)$ is the effective integrated interaction between a point of the liquid-vapor interface with the homogeneous, planar substrate, and it is termed interface potential. In this special case, the free energy functional (3.27) takes the form (up to a constant)

$$
F[h]=L_{y} \int \mathrm{d} x \sqrt{1+\left(\partial_{x} h\right)^{2}}[\gamma+g(h)]
$$

where $L_{y}$ denotes the system dimension parallel to the cylinder axis. In the limit that the equilibrium contact angle is small, one can adopt a long-wavelength approximation (or small-gradient expansion)

$$
F[h] \approx L_{y} \int \mathrm{d} x\left[1+\frac{1}{2}\left(\partial_{x} h\right)^{2}+\cdots\right][\gamma+g(h)]
$$

We obtain the equilibrium shape of the droplet by minimizing this free energy functional subject to the constraint of fixed droplet volume

$$
V_{\text {drop }}=L_{y} \int \mathrm{d} x h(x)=\text { const }
$$

yielding the condition

$$
\pi(x)=-\frac{1}{L_{y}} \frac{\delta F}{\delta h(x)}=\lambda
$$

\footnotetext{
${ }^{2}$ If there exist additional long-ranged interactions, $V_{\mathrm{lr}}$, between the liquid and the solid, then one has the additional contribution $F_{\text {lr }}=\int_{\mathrm{L}} \mathrm{d}^{3} \mathbf{r} \int_{\mathrm{S}} \mathrm{d}^{3} \mathbf{r}^{\prime} V_{\operatorname{lr}}\left(\left|\mathbf{r}-\mathbf{r}^{\prime}\right|\right)$. Writing $V_{\operatorname{lr}}(|\mathbf{r}|)=\nabla \cdot \mathbf{r} \Phi_{\operatorname{lr}}(|\mathbf{r}|)$, we obtain for the long-range contribution $F_{\text {Ir }}=\int_{\text {SLULV }} \mathrm{d} \mathbf{S} \cdot \int_{\mathrm{S}} \mathrm{d}^{3} \mathbf{r}^{\prime}\left(\mathbf{r}-\mathbf{r}^{\prime}\right) \Phi_{\operatorname{lr}}\left(\left|\mathbf{r}-\mathbf{r}^{\prime}\right|\right)=\int_{\text {SLULV }} \mathrm{d} \mathbf{S} \cdot \mathbf{e}_{x} g_{\operatorname{lr}}(h)$ with $g_{\operatorname{lr}}(h)=\int_{\mathrm{S}} \mathrm{d}^{3} \mathbf{r}^{\prime} \mathbf{e}_{x} \cdot\left(\mathbf{r}-\mathbf{r}^{\prime}\right) \Phi_{\operatorname{lr}}\left(\left|\mathbf{r}-\mathbf{r}^{\prime}\right|\right)=L_{y} \int \mathrm{d} x g_{\operatorname{lr}}(h)+$ const.
} 
where $\lambda$ is a Lagrange multiplier constraining the droplet volume. Using Eq. (3.29) we obtain

$$
\begin{aligned}
\pi(x) & =-\sqrt{1+\left(\partial_{x} h\right)^{2}}\left[\partial_{h} g\right]+\partial_{x}\left(\frac{\partial_{x} h}{\sqrt{1+\left(\partial_{x} h\right)^{2}}}[\gamma+g(h)]\right) \\
& =\frac{\partial_{x x} h[\gamma+g(h)]}{\left[1+\left(\partial_{x} h\right)^{2}\right]^{3 / 2}}-\frac{\partial_{h} g}{\sqrt{1+\left(\partial_{x} h\right)^{2}}}
\end{aligned}
$$

In the limit of small contact angles, $\left|\partial_{x} h\right| \ll 1$, this equation adopts the form

$$
\pi(x)=\partial_{x x} h[\gamma+g(h)]-\partial_{h} g
$$

The pressure (3.33) consists of two contributions: the curvature pressure, where $\kappa_{\text {full }}=$ $\frac{\partial_{x x} h}{\left[1+\left(\partial_{x} h\right)^{2}\right]^{3 / 2}}$ is the curvature and $\gamma+g(h)$ is the effective tension of the interface a distance $h$ away from the solid substrate, respectively. The Derjaguin (or disjoining) pressure $\Pi(h)=-\partial_{h} g(h)$ models wettability $[80,81]$. The dimensionless ratio $g(h) / \gamma$ dictates the shape of a drop in the continuum model and it is this parameter that we have extracted from the particle-based model in Sec. 3.1.5.

A spatially non-uniform pressure, $\pi(x)$, gives rise to a flow of liquid inside the film. Using the Navier-Stokes equation and employing the long-wavelength approximation $[59,89,90]$, one obtains

$$
\partial_{t} h=-\partial_{x} \Gamma=-\partial_{x}\left\{Q(h) \partial_{x} \pi(x)\right\}
$$

Here $Q(h)=h^{3} / 3 \eta$ is the mobility, $\eta$ is the dynamic viscosity of the liquid. Note, that $\Gamma$ is a flux that is written as the product of a mobility and a pressure gradient. Eq. (3.35) with (3.34) is sometimes called a thin-film or lubrication model.

The equation describing stationary solutions may either be obtained by directly minimizing the functional $F[h]$ according to Eq. (3.32) or, alternatively, one sets $\partial_{t} h=0$ in Eq. (3.35) and integrates twice taking into account that $\Gamma=0$ in the steady state. Here we use numerical continuation techniques [91] to solve the resulting ordinary differential equation as a boundary value problem on a domain of size $L$ with boundary conditions such that the center of the resulting drop solution is positioned on the right boundary $(x=L)$ and on the left boundary $(x=0)$ the profile approaches a precursor film. The volume is controlled by the integral condition, Eq. 3.31. Figure 3.10(a) presents typical drop profiles for various volumes whereas Fig. 3.10(b) gives the maximal drop height as a function of drop volume. Note, that there exists a minimal droplet volume $V_{\text {sn }}$ given by the saddle-node bifurcation in Fig. 3.10(b). If one decrease the volume below $V_{\mathrm{sn}}$, the droplet collapses, i.e., it changes discontinuously into a flat film. The transition is hysteretic (first order) as the primary bifurcation at $V_{\mathrm{c}}$ is subcritical. 
(a)
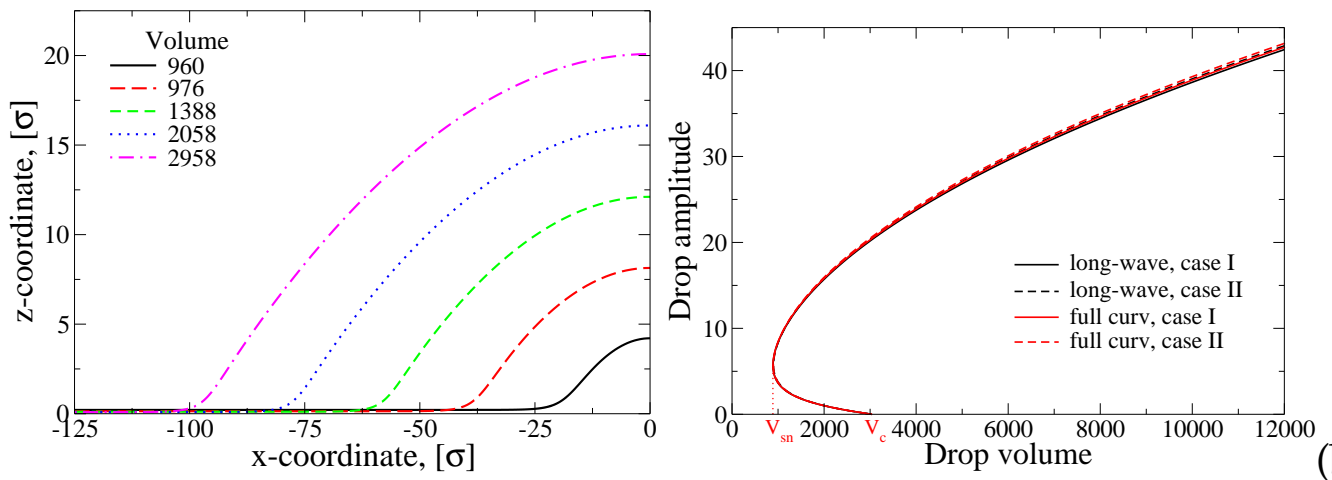

Figure 3.10: (a) Shown are selected half-profiles of droplets at volumes as given in the legend and (b) the bifurcation diagram presenting the drop height in dependence of the drop volume. Calculations are performed with (i) the full curvature, i.e., Eq. (3.32) with (3.33), and (ii) the long-wavelength curvature, i.e., Eq. (3.32) with (3.34). Case I and II refer to usage of only $\gamma$ or the full $\gamma+g(h)$ as prefactor of curvature, respectively. The profiles in panel (a) are obtained with case I for full curvature. The volume is controlled through appropriately adapting the Lagrange multiplier $\lambda$ at fixed domain size $L=4000$. The employed disjoining pressure and interfacial tensions are extracted from MD simulations at $\epsilon_{\mathrm{s}}=0.81 \epsilon$ (equivalent to an equilibrium contact angle of $\theta_{\mathrm{E}}=23.57^{\circ}$, for details see below section 3.2.2).

From Eqs. (3.33), (3.34) and (3.35) we conclude that the information needed in the continuum model is the interfacial tension $\gamma$ and interface potential $g(h)$ (or, equivalently, the disjoining pressure $\Pi(h)$ ). The parameter-passing scheme is illustrated in Fig. 3.11. The reliability of the scheme is tested by comparison of droplet profiles in Sec. 3.2.3.

\subsubsection{Contact angles of droplets in MD and CD. Equilibrium contact angle}

In the following we will compare the shape of droplets obtained from the particlebased model and the continuum description. This comparison focuses on droplets with small contact angles $\leq 50^{\circ}$ obtained in the particle-based model for the strengths of solid-liquid interaction close to the wetting transition $\left(\epsilon_{\mathrm{s}}=0.75 \epsilon\right.$ to $\left.0.82 \epsilon\right)$. Different numbers of polymer chains are used to create cylindrical $2 \mathrm{~d}$ droplets ( $3 \mathrm{~d}$ ridges) of varying volumes and hence heights. The spanned dimension of the simulation domain is smaller that the other lateral one, namely $L_{y} \approx 26 \sigma<L_{x}$, whereas $L_{x}$ is dictated by the size of the drop and its contact angle. A repulsive wall is placed far above the droplet.

Data are sampled with a frequency of $4000 \mathrm{MD}$ steps (i.e., the time interval between two snapshots is $4000 \cdot \Delta t=4000 \cdot 0.005 \tau=20 \tau)$. This time interval between two samples corresponds to the Rouse relaxation time for a similar polymer liquid $\tau_{R}=25.6 \pm 5 \tau$ [27]. For small droplets (up to 600 chains) the sampling lasted $2 \times 10^{6}$ steps, whereas for bigger ones (up to 9600 chains) this interval was increased up to $10^{7}$ steps, because large 


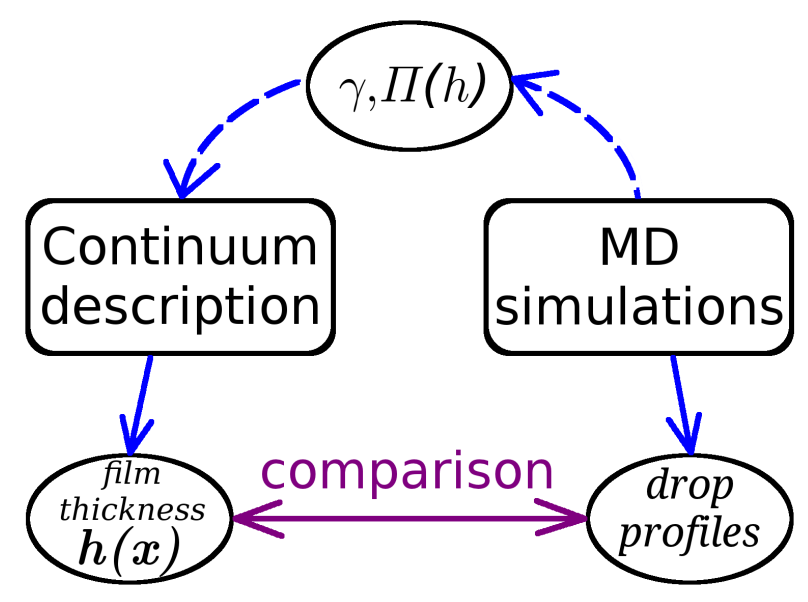

Figure 3.11: Shown is the parameter-passing scheme to bridge two representations: particle-based description and continuum model (thin-film equation). The parameters needed to be passed are calculated in MD simulations of the particle-based description and passed to the continuum model. Then, droplet profiles as obtained by the two approaches are compared, see Sec. 3.2.3.

fluctuations of the droplet shape occur. As a result, every density profile is obtained by averaging over 500 (small drops) or 2500 (large drops) snapshots. To extract the droplet shape and measure the contact angle, we use a set of density profiles obtained in 10 independent runs. In total, all large droplets are simulated over $10^{8}$ steps.

The resulting cylindrical droplet snapshots are cut into slices along the invariant $y$ direction. An average over these slices with respect to its center-of-mass position results in the average number density profile in the $(x, z)$ plane. A two-dimensional drop profile is extracted by localizing the solid-liquid and liquid-vapor interfaces by the crossing criterion for the density as $\rho_{\text {int }}=\left(\rho_{\text {liq }}+\rho_{\text {vap }}\right) / 2$. Examples of profiles are presented in Fig. 3.12. The resulting profiles are then compared to the ones extracted from the parameterized continuum models, which are also presented in Fig. 3.12.

One popular characteristics of the drop shape is the contact angle, because it is related to the balance of interfacial tensions at the three-phase contact line of a macroscopic drop. For finite-sized drops, however, the contact angle is not uniquely defined: (i) One may define a mesoscopic contact angle $\theta_{\text {mes }}$ as the slope at the inflection point of the droplet profile. This is often done in thin film models [60, 92], however, the steepest slope obtained in this way may not coincide with the (larger) macroscopic contact angle even in the limit of large drop size [93]. This corresponds to the distinction of macroscopic and microscopic contact angle in Ref. [94]. Moreover, in the particle-based model, the inflection point may be located very close to the three-phase contact line where liquid-like layering effects of the particle fluid may occur and af- 


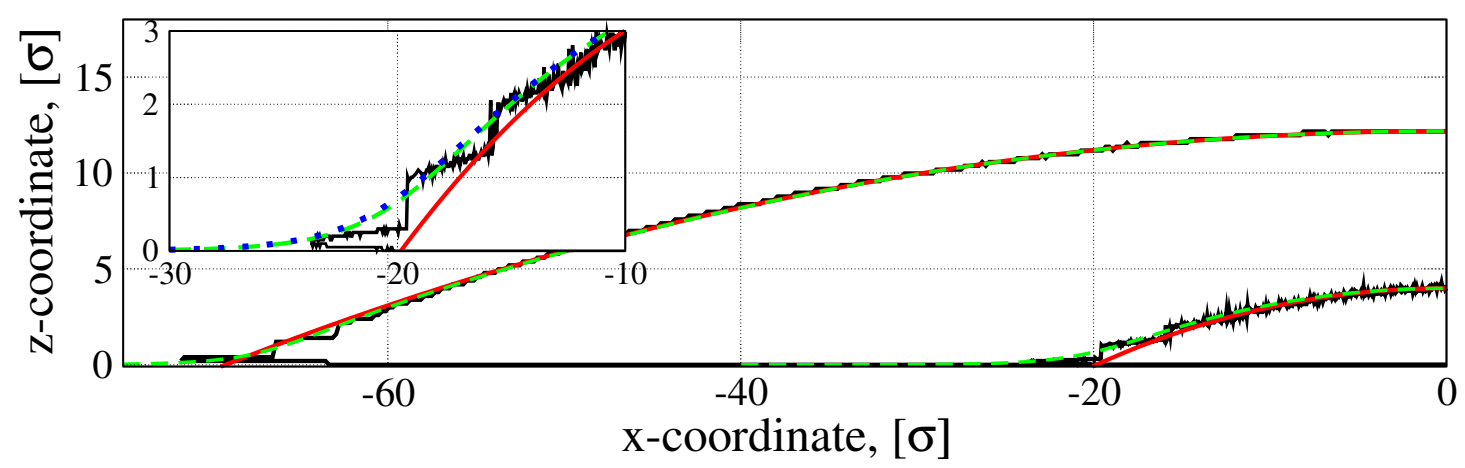

Figure 3.12: Profiles of two-dimensional droplets obtained by cutting cylindrical droplets obtained in MD simulations [solid noisy line (black)] for the case $\epsilon_{\mathrm{s}}=0.82 \epsilon$ for two values of $h_{\max }(4.046 \sigma$ and $12.181 \sigma)$. The corresponding spherical cap fit is given as solid smooth line (red). The MD drops are compared with results of the continuum model Eq. (3.32) with the full curvature [Eq. (3.33)] and in long-wavelength approximation [Eq. (3.34)] that are given as dashed (green) and dotted (blue) lines, respectively. The inset shows a zoom into the three-phase contact line region of the smaller droplet.

fect the drop profile.(ii) Alternatively, one may define a spherical cap contact angle by approximating the drop profile by a spherical cap profile with a minimal radius of curvature $R=-1 / \kappa$, i.e., using the curvature at $h_{\max }$. The resulting contact angle is $\theta_{\mathrm{sph}}=\arccos \left(1-h_{\max } / R\right)$. In the profiles extracted from the particle-based model, we extract $\theta_{\text {sph }}$ by only considering the central part of the drop to define the curvature. In this way, the calculation is not perturbed by liquid-like layering effects or by the shortrange interface potential that distorts the liquid-vapor interface close to the three-phase contact line. The height of the drop is determined as the difference of the highest point of the spherical cap and the position of the solid-liquid interface ${ }^{3}$. $\theta_{\text {sph }}$ converges to the proper macroscopic contact angle in the limit of large drop size, but may misrepresent the shape and volume of small droplets.

For the continuum model, the two angles $\theta_{\text {sph }}$ and $\theta_{\text {mes }}$ are illustrated in Fig. 3.13 that shows two droplet profiles $h(x)$ as obtained from Eqs. (3.32) with (3.33), their approximated spherical cap profiles and the tangents of $h(x)$ at the point of steepest slope (yielding $\theta_{\text {mes }}$ ) and of the spherical cap profile at the point where it crosses the precursor height (giving $\theta_{\text {sph }}$ ). One clearly notes that the two measures differ, and that the difference decreases with increasing droplet size. We will see below that the two measures do not converge even for very large drops. In the following we focus on the spherical cap contact angle $\theta_{\text {sph }}$.

The resulting contact angles for drops of various sizes are presented for different $\epsilon_{\mathrm{s}}$

\footnotetext{
3 There are different strategies of measuring the contact angle with the spherical cap approximation: direct geometric measurements [28, 95], estimation from the center of mass position [27] or from the volume of the droplet. In our MD simulations, we define a contact angle by the geometrical method. Other methods give a similar result as all of them assume the spherical shape of the droplet.
} 


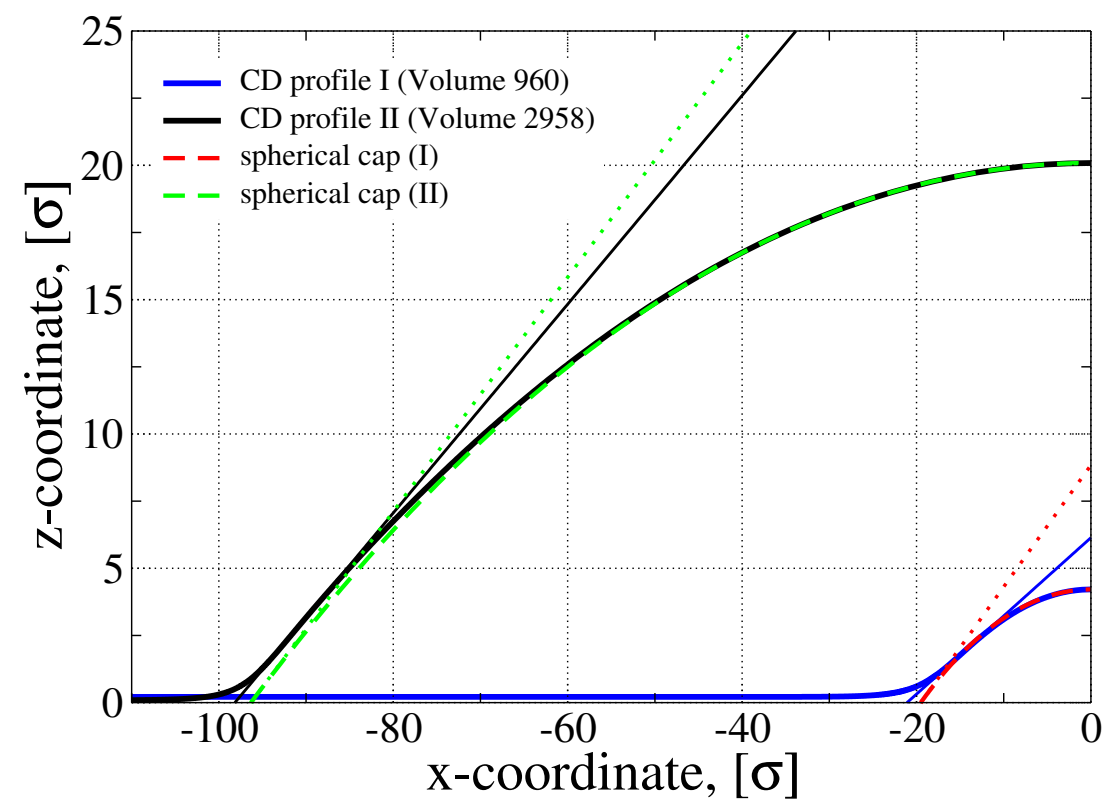

Figure 3.13: Droplet profiles as obtained from continuum description (CD) with full curvature (heavy solid lines) for $\epsilon_{\mathrm{s}}=0.81 \epsilon$ and drop height (a) $H=4$ and (b) $H=20$. Also shown are the spherical caps as obtained from the curvature at the drop maxima (heavy dotted lines), and the tangent lines at the point of the steepest slope of the profile (thin solid line), and the tangent line of the spherical cap profile at precursor height (thin dotted line). Drop height $H$ is defined as difference of height at maximum and precursor height.

as open square symbols in Fig. 3.14. Overall, they agree well with the prediction of Eq. (3.14) that is given as horizontal dashed black line (with the standard deviation indicated as a grey shaded region). Corresponding results for the contact angle obtained from the continuum model, employing the long-wavelength approximation for the curvature, Eq. (3.34), and with the full curvature, Eq. (3.33), are given as well. The results for both are shown as solid (case I: only $\gamma$ as prefactor of curvature) and dashed (case II: the full $\gamma+g(h)$ as prefactor of curvature ) lines of different colors depending on the angle shown $\left(\theta_{\text {mes }}\right.$ or $\left.\theta_{\text {sph }}\right)$. Note that both curvature models result in identical results for $\theta_{\text {sph }}$ because $\partial_{x} h=0$ at the apex of the drop. This is not the case for $\theta_{\text {mes }}$.

The angle $\theta_{\text {sph }}$ obtained in the continuum approach agrees well with the result of the MD simulations. This is particularly true for case I (only $\gamma$ as prefactor of curvature) where $\theta_{\text {sph }}$ converges for large drops to the value obtained with the Young's equation. The deviations of case II from case I are small over the entire thickness range for $\epsilon_{\mathrm{s}}=$ $0.82 \epsilon, \epsilon_{\mathrm{s}}=0.81 \epsilon$ and $\epsilon_{\mathrm{s}}=0.80 \epsilon$, but rather large for $\epsilon_{\mathrm{s}}=0.75 \epsilon$. Note, that $\theta_{\text {mes }}$ does not agree well with the macroscopic angle obtained in the MD simulations. Within long-wavelength approximation it is always at least some percent smaller than $\theta_{\text {sph }}$ (more so for small droplets). The angle $\theta_{\text {mes }}$ obtained with the full curvature differs less from $\theta_{\mathrm{sph}}$, the difference becomes less than one percent for large drops. For both curvature models, $\theta_{\text {mes }}$ always decreases monotonically with decreasing drop size. All 


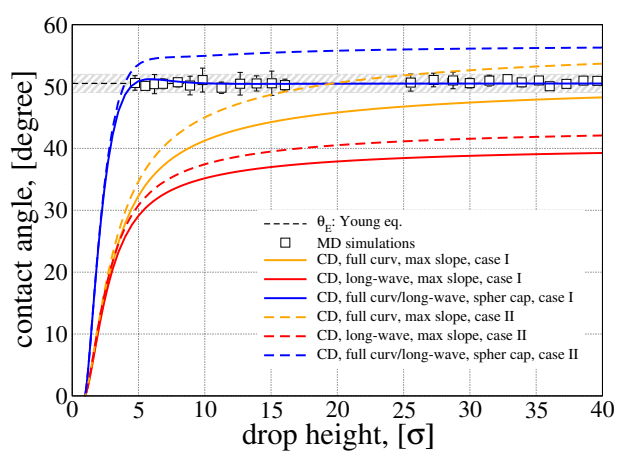

(a)

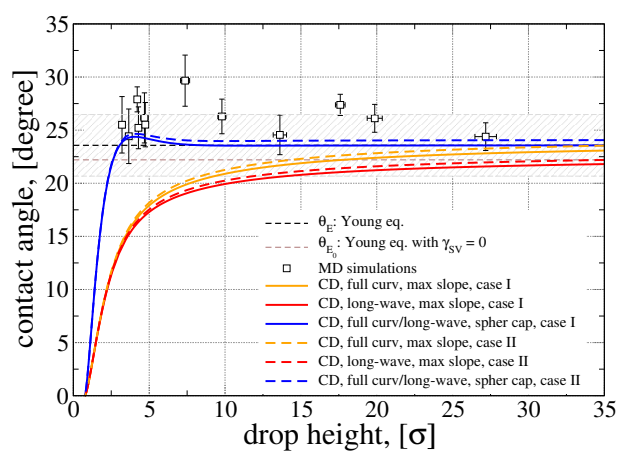

(c)

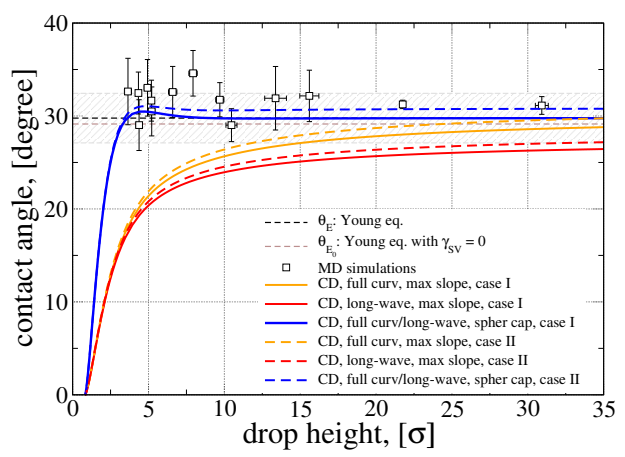

(b)

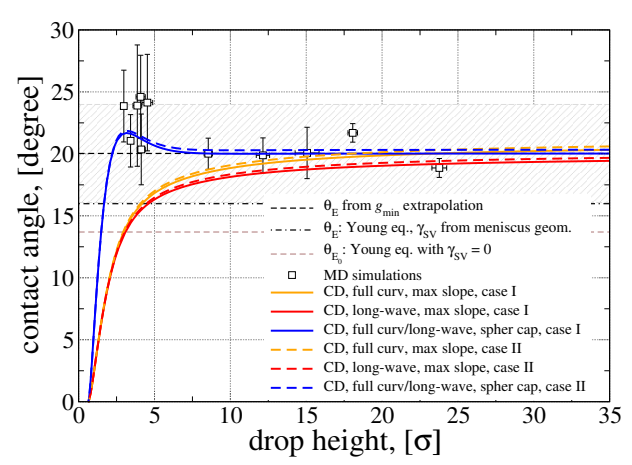

(d)

Figure 3.14: Contact angles $\theta$ of droplets of different sizes as a function of droplet height. Panels (a), (b), (c) and (d) give results at solid-liquid interaction strengths of $\epsilon_{\mathrm{s}}=0.75 \epsilon$, $\epsilon_{\mathrm{s}}=0.80 \epsilon, \epsilon_{\mathrm{s}}=0.81 \epsilon$ and $\epsilon_{\mathrm{s}}=0.82 \epsilon$, respectively. Square symbols correspond to the contact angle measured in MD simulations, using a spherical cap approximation of the droplet profile. Dotted and dashed thin horizontal lines correspond to the values $\theta_{\mathrm{E}_{0}}$ and $\theta_{\mathrm{E}}$ obtained from the Young equation with and without accounting for the measured solidvapor interfacial tension $\gamma_{\mathrm{SV}}$, respectively. Shaded zones show the standard deviation of $\theta_{\mathrm{E}}$. Panel (d) additionally shows as a dot-dashed horizontal line the value of $\theta_{\mathrm{E}}$ as extracted from the meniscus geometry. The thick solid (case I) and dashed (case II) curves (orange, red and blue) in panels (a) to (d) represent the spherical cap contact angle $\theta_{\text {sph }}$ and the mesoscopic steepest slope contact angle $\theta_{\text {mes }}$ obtained from the continuum description (CD) with full and long-wavelength curvature [Eq. (3.32) with Eqs. (3.33) or (3.34)], as indicated in the legend. Case I and II refer to using of only $\gamma$ or the full $\gamma+g(h)$ as prefactor of curvature, respectively [cf. Eqs. (3.34) and (3.33)].

these statements apply for the respective relation between the various curves in case I equally as in case II. The various angles calculated in case I are always slightly below the ones obtained in case II.

Inspecting Fig. 3.14, one notes a number of further details that warrant to be highlighted: (i) A common feature of the particle-based model for $\epsilon_{\mathrm{s}} \geq 0.80 \epsilon$, shown in Figs. 3.14b - 3.14d, is the overshooting of the values of contact angles at thicknesses $h \approx 3-7 \sigma$. This effect can also be observed in the spherical cap contact angle ob- 
tained from the continuum models. It indicates that the product of drop height $h_{\max }$ and curvature at the drop apex $\kappa_{\max }$ is not a constant any more, instead $\left|h_{\max } \kappa_{\max }\right|$ first increases with increasing volume (before decreasing again). (ii) Another detail one notices is the importance of the solid-vapor interfacial tension, $\gamma_{\mathrm{SV}}$, measured in Sec 3.1.4. At $\epsilon_{\mathrm{s}}=0.75 \epsilon$ it equals zero and at $\epsilon_{\mathrm{s}}=0.80 \epsilon$ the macroscopic contact angles are almost the same if one neglects $\gamma_{\mathrm{SV}}$ or properly accounts for it (cf. the dotted and dashed horizontal lines in Fig. 3.14b, respectively). However, the difference between the two approaches becomes increasingly important with increasing $\epsilon_{\mathrm{s}}$, i.e. decreasing contact angle (Figs. 3.14c and 3.14d). Taking a non-zero $\gamma_{\mathrm{SV}}$ into account becomes crucial close to the wetting transition. There, for rather small values of the contact angle (about $15-20^{\circ}$ ) the difference is of the order of $20-40 \%$ and accounts for $2-6^{\circ}$. The difference can lead to an incorrect prediction of the contact angle if one assumes $\gamma_{\mathrm{SV}}=0$ in the particle-based model.

Finally, we note that the error bars of the contact angles $\theta$ measured in MD simulations using a spherical cap approximation of the droplet profile (open squares in Figs. 3.14a to $3.14 \mathrm{~d}$ ) are quite large. They increase with decreasing contact angle even in absolute terms. Several possible explanations exist for this behavior: (i) In the vicinity of the wetting transition, there are strong capillary waves on the surface of the droplet (particularly close to the three phase contact line) [96]. (ii) The crossing criterion we apply to define the profile of the drops $\left(\rho_{0}+\rho_{V}\right) / 2$ is not a unique choice. There are other possibilities to define the local interface position based, e.g., on $10-90 \%$ or $20-80 \%$ rules that may work somewhat better close to the wetting transition (cf. $[42,95,97,98]$ ).

\subsubsection{Comparison of profiles of droplets obtained by CD and particle-based model}

Next, we compare the drop profiles as obtained from the particle-based model and the continuum description. For the case of a rather small contact angle, $\epsilon_{\mathrm{s}}=0.82 \epsilon$, Fig. 3.12 gives results for a very small droplet of $h_{\max }=4.046 \sigma$ and a larger one with $h_{\max }=12.181 \sigma$. The layering effects of the particle-based model are rather independent of droplet size. Obviously, the layering of the particle-based model is not captured by the continuum model, however, its predictions go smoothly through the steps of the profile and always lay between the lateral end points of the steps. At the center of the drop, the spherical-cap fit to the particle-based model and the continuum results, obtained with Eq. (3.32) with the full curvature (Eq. (3.33)) as well as in long-wavelength approximation (Eq. (3.34)), nicely agree with each other. As cases I and II can not be distinguished by eye alone we have only included case I.

Differences between long-wavelength and full curvature and the results of the particlebased model are only visible in the contact line region. There, the spherical cap is not 


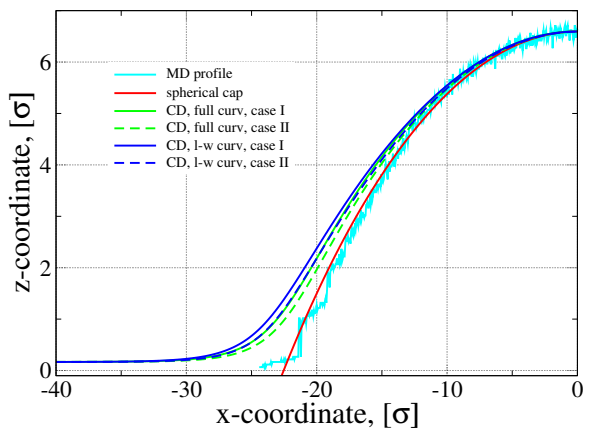

(a)

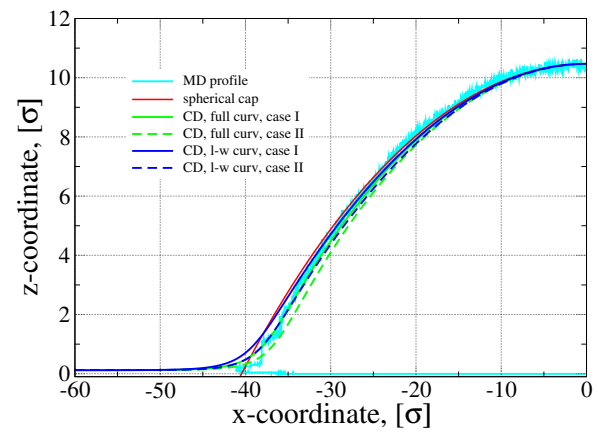

(b)

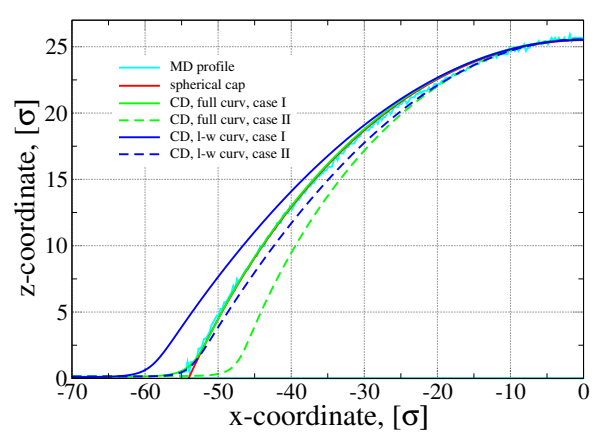

(c)

Figure 3.15: Droplet profiles obtained in MD simulations and with continuum models are compared for the case $\epsilon_{\mathrm{s}}=0.80 \epsilon$, apex heights (a) $h_{\max }=6.594 \sigma$ and (b) $h_{\max }=10.469 \sigma$ and (c) $\epsilon_{\mathrm{s}}=0.75 \epsilon$ and apex height $h_{\max }=25.494 \sigma$. The solid curves (light blue) give the liquid-vapor interface as obtained in the MD simulation, while the gray solid curves (red) give the corresponding spherical cap fit. Results of the continuum description (CD) Eq. (3.32) with the full curvature (Eq. (3.33) - green curves) and in long-wave approximation (Eq. (3.34) - dark blue curves) are shown for cases I and II as solid and dashed curves, respectively. For details see main text.

a good fit to the particle-based model. The two continuum models nearly coincide, implying that the long-wavelength approximation for static droplets is still very good for contact angles around $20^{\circ}$. In the contact line region, they seem to represent a better approximation to the particle-based model than the spherical cap. One should actually expect this, as the continuum models incorporate the Derjaguin pressure as measured in the particle-based model. One may conclude that within its limitations the continuum model describes the profiles rather well if it incorporates the interfacial tensions and Derjaguin pressure from particle-based model.

The situation differs for larger contact angles as obtained for $\epsilon_{\mathrm{s}}=0.80 \epsilon$ and shown in Fig. 3.15(a) and (b): (i) The deviation from the spherical-cap approximation is more significant than for the smaller contact angle and (ii) the continuum model fails to describe the simulation data for the smaller droplet size. The difference between the predictions of the different versions of the continuum description is small compared to the 
deviation between the continuum models and the particle-based model. Therefore, the reason of the discrepancy is not rooted in the different approximations of the curvature. We note that interface fluctuations in a small droplet are strongly suppressed. Therefore, one should rather use the bare interface potential than the one extracted from a laterally extended film. Since the bare interface potential has a smaller range than the renormalized one [61], we expect the profile of a small droplet to be better approximated by a spherical-cap shape than that of a large one, which is indeed consistent with the simulation data. Out of the same reason, the predictions of the continuum model are more accurate for the larger drop than for the smaller one because it uses the renormalized interface potential as input. This rational explains why the predictions of the continuum model systematically deviate from the results of the particle-based model for small droplet size. For the large droplet, in contrast, the continuum model succeeds in describing the deviations from the spherical cap shape, which is larger than for small contact angles. The profile of the particle-based model lays right in the middle of the predictions of the continuum models. The one that fits best is the case I with full curvature. Therefore, we conclude that even for contact angles of about $30^{\circ}$ all models agree fairly well with the particle-based simulations provided the appropriate interface potential is used.

Finally, we compare the profiles with a rather large contact angle as obtained for $\epsilon_{\mathrm{s}}=$ $0.75 \epsilon$ and shown in Fig. 3.15(c). For the comparison we use a large droplet with $h_{\max }=$ $25.494 \sigma$. The difference between the various versions of the continuum models is clearly seen not only at the contact line but over the entire droplet profile. The best agreement with the particle-based model is achieved for case I with full curvature; all other versions differ more significantly. Therefore, we conclude that for contact angles of about $50^{\circ}$ only the model with full curvature agrees well with the particle-based model, while the long-wavelength approximation is not valid anymore. It is not advisable to apply at $\theta_{\mathrm{E}}=50^{\circ}$ where it predicts a contact angle $\theta_{\text {mes }}$ that is $20 \%$ lower.

\subsection{Hydrodynamic properties of non-equilibrium polymer liq- uids}

This section deals with fundamental problems of solid-liquid interface description. Employing non-equilibrium molecular dynamics simulations (NEMD) with DPD thermostat to preserve correct hydrodynamics, we study liquid flows. While in a macroscopic world the no-slip condition is usually observed, where the layer of the liquid in direct contact with flat solid assumes the velocity of the solid itself $[99,100]$, the microscopic picture is richer (cf. Fig. 3.16). Partial and even full slippage (plug flow) are often observed at this level $[10,101]$. Since our particle-based simulations are micro- 


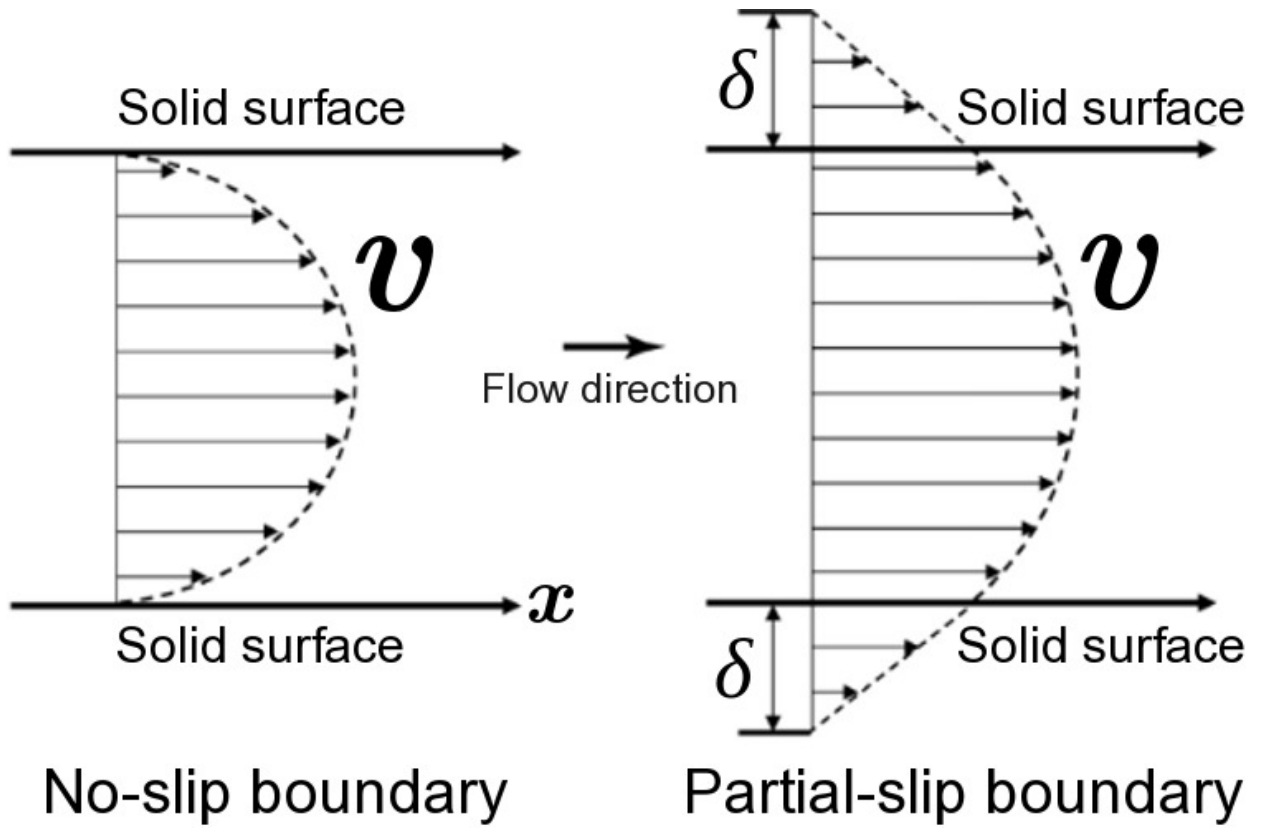

Figure 3.16: Shown is a comparison of a macroscopic situation with no-slip boundary condition, $\delta=0$, and a microscopic surface-induced deviations from it (partial slippage). The slip length here is defined as the distance from the solid-liquid interface to the no-slip position.

scopic, they are a suitable tool for investigating the boundary problem induced by the solid-liquid interface.

In the following, we apply the macroscopic concept of boundary conditions to the microscopic velocity profiles of liquid passing the solid. The definition of slip length as the distance from the solid-liquid interface to the position, where interpolated profile of the liquid reaches the velocity of the wall (no-slip position) [102], is not free of ambiguity. The reason is that such slippage depends on the type of the flow. Hence, it can not serve as the boundary condition in macroscopic sense. Instead, an approach based on macroscopic Navier's boundary condition [103] is presented that is independent of the type of the flow. In this method, the solid-liquid boundary is presented in terms of slip length, $\delta$, and hydrodynamic boundary position, $z_{\mathrm{h}}$, at which the condition is applied.

\subsubsection{Navier's boundary condition}

In order to study flow past solid surfaces, we move the two apposing substrates into opposite directions with a small absolute velocity $v_{\text {wall }}^{\mathrm{C}}=0.075 \sigma / \tau$ (Couette flow) as shown in Fig. 3.17a. The average shear rate is defined as a ratio of the velocity of the wall $v_{\text {wall }}^{\mathrm{C}}$ to the width of the channel $L_{z}$ and, in our simulations, it is of the order of $\dot{\gamma} \sim 5 \cdot 10^{-3} \tau^{-1}$. The product of the shear rate and the Rouse relaxation time, $\tau_{R}$, of the 


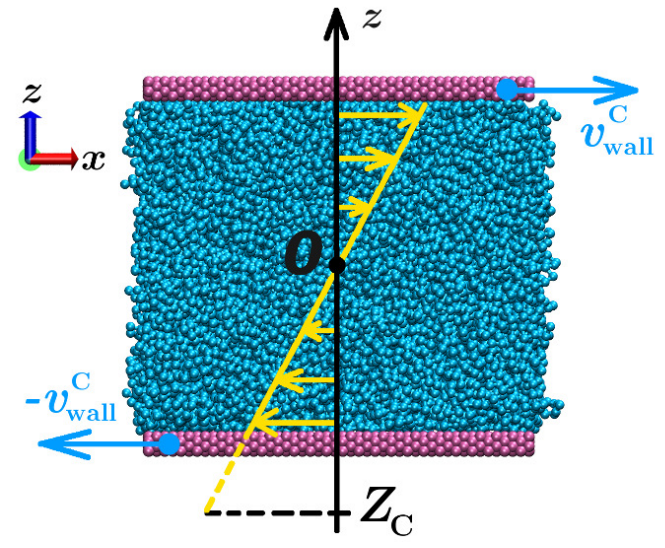

(a)

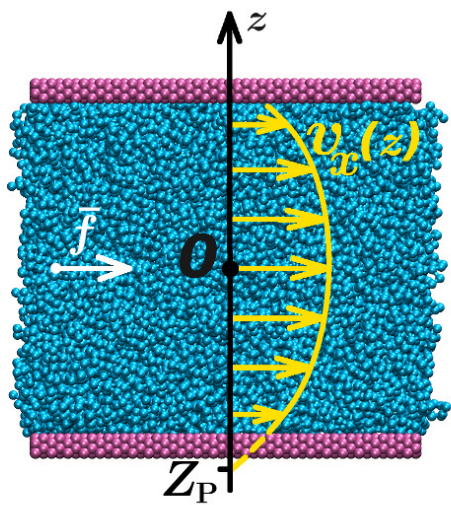

(b)

Figure 3.17: (a) Couette flow created in the system with flat substrates. Two walls are moved into opposite directions with absolute velocities $v_{\text {wall }}^{C}$. The origin of the $z$-axis is taken in the middle of the channel (black). The linear velocity profile is sketched by the arrows of different size. The interpolated profile reaches the velocity of the wall at the coordinate $Z_{C}$. (b) The flow created by application of a body force $\vec{f}$ (white) onto the particles of the liquid. The parabolic velocity profile (arrows) is interpolated to the velocity of the wall $\left(v_{\text {wall }}^{\mathrm{P}}=0 \sigma / \tau\right)$ and reaches it at $Z_{\mathrm{P}}$.

polymers defines the Weissenberg number, $\mathrm{Wi}=\dot{\gamma} \tau_{\mathrm{R}}$. Since $\mathrm{Wi} \approx 0.13 \ll 1$, we do not expect the weak flow to significantly perturb the molecular conformations.

As every boundary problem, the flow of liquid in the vicinity of the solid can be expressed in terms of effective parameters that are independent from the way the flow is set up. In the static case we have seen that Young's equation (3.14), manifesting the balance of surface and interfacial tensions (free energies) at the contact line, described the droplet at equilibrium. An analogy in dynamic case can be found in the balance of stresses. This condition was formulated by Navier [103] and it balances the viscous and frictional stresses at the solid-liquid boundary

$$
\left.\eta \frac{\partial v_{x}}{\partial z}\right|_{z=z_{\mathrm{h}}}=\lambda v_{s},
$$

where $\eta$ is the viscosity of the liquid, $v_{x}$ is the $x$ component of the velocity of the liquid parallel to the substrate. $\lambda$ is the friction at the solid-liquid boundary, and the slip velocity, $v_{s}=v_{x}\left(z_{\mathrm{h}}\right)$, represents the velocity of the liquid at the hydrodynamic boundary $z_{\mathrm{h}}$. The slippage is then defined as the ratio

$$
\delta=\frac{\eta}{\lambda}
$$

In order to serve as a boundary condition of a continuum description (i.e., the NavierStokes equation), changes of the liquid structure at the surface are ignored, and $\eta$ is interpreted as the shear viscosity of the liquid in the bulk, $\eta=5.3 \pm 0.1 \sigma^{2} / \sqrt{m \epsilon}$ [27]. 
By the same token, $v_{x}(z)$ is interpreted as the macroscopic velocity profile that obeys the continuum description and that we can extract from simulations by extrapolating the linear profile of Couette flow towards the substrate, $z=z_{\mathrm{h}}$.

Thus, Navier's hydrodynamic boundary condition, Eq. (3.36), parameterizes the flow past the surface by two effective material constants of the surface [27, 104-107]: the hydrodynamic position, $z_{\mathrm{h}}$, at which the boundary condition to the macroscopic continuum description is applied and the slip length, $\delta=\eta / \lambda$. The flow of simple liquids over flat substrates is often described by the no-slip boundary condition, $\delta=0$ [108], and the hydrodynamic position, $z_{\mathrm{h}}$, often coincides with the location of the sharp solid-liquid interface, as intuitively expected. Finite slip has been observed in complex liquids [27, 34, 70, 101, 106] or at specific substrates: superhydrophobic [109-114] and chemically patterned ones $[115,116]$.

Therefore we expect a finite slip length, $\delta$, and the effective location, $z_{\mathrm{h}}$, at which the hydrodynamic boundary conditions is to be applied, is not obvious. Since Navier's boundary condition features two independent parameters, we use two flow profiles: Couette flow, generated by moving the surfaces, and Poiseuille flow, generated by applying an external body force on the liquid. The linear and parabolic velocity profiles expected from the macroscopic continuum model for Couette and Poiseuille flow, respectively, are illustrated in Fig. 3.17.

We chose the origin of the coordinate system at the center of the film. The macroscopic velocity profile is linear $v_{x}^{\mathrm{C}}(z)=a z+b$ for Couette flow, and the constants $a$ and $b$ are determined from the boundary conditions $v_{x}^{C}(0)=0$ and $v_{x}^{C}\left(Z_{C}\right)=-v_{\text {wall }}^{C}$, where $Z_{C}<0$ is the position where extrapolation of the linear, macroscopic velocity profile equals to the velocity of the bottom wall $-v_{\text {wall }}^{C}$ as shown in Fig. 3.17a. The resulting velocity profile is therefore

$$
v_{x}^{\mathrm{C}}(z)=-\frac{v_{\mathrm{wall}}^{\mathrm{C}}}{Z_{\mathrm{C}}} z
$$

According to the macroscopic Navier-Stokes equation, the velocity profile of the flow generated by an external body force has a parabolic shape, $v_{x}^{\mathrm{P}}(z)=-\frac{\rho_{\text {coex }} f}{2 \eta} z^{2}+A z+B$, where $\rho_{\text {coex }}=0.786 \sigma^{-3}$ is the number density of the liquid and $\eta$ denotes its viscosity. $A=0$ by symmetry. When we express the constant $B$ by the boundary condition $v_{x}^{\mathrm{P}}\left( \pm Z_{\mathrm{P}}\right)=0$, we obtain:

$$
v_{x}^{\mathrm{P}}(z)=\frac{\rho_{\text {coex } f}}{2 \eta}\left(Z_{\mathrm{P}}^{2}-z^{2}\right) .
$$

$Z_{\mathrm{P}}$ is the coordinate, where the extrapolated parabolic velocity profile of the liquid reaches the velocity of the wall as illustrated in Fig. 3.17b. Velocity profiles are fitted by Eqs. 3.38 and 3.39 at the center of the film excluding the near-substrate region. Applying the hydrodynamic boundary condition, Eq. (3.36), to the macroscopic profiles, 


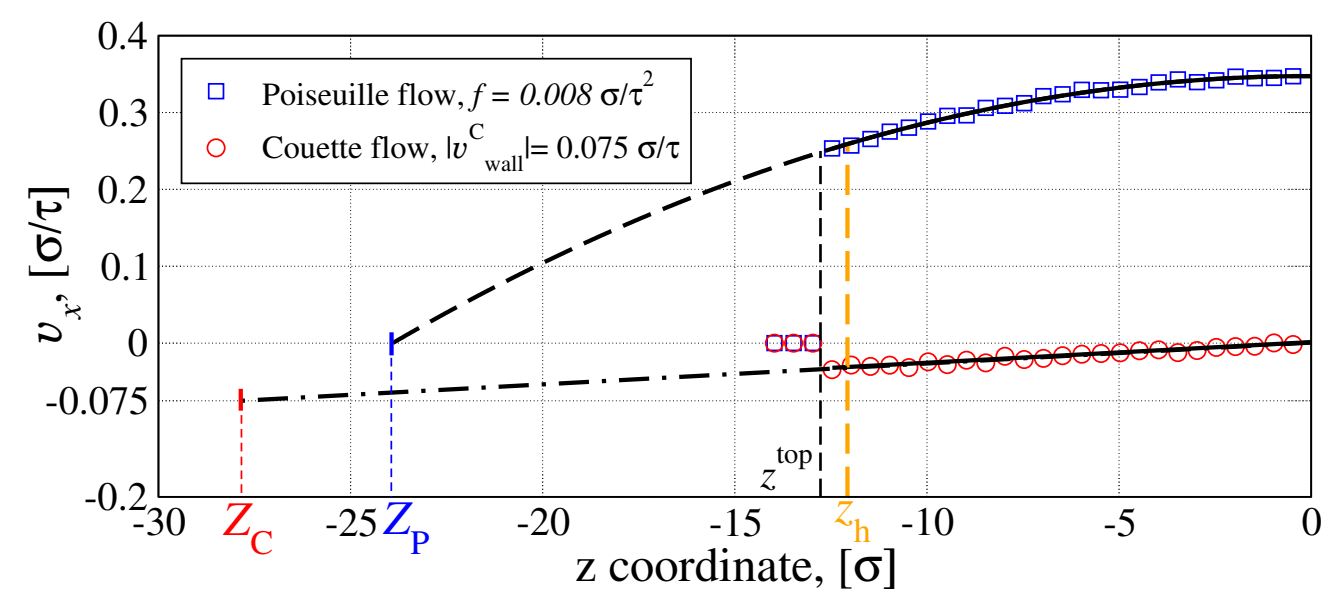

Figure 3.18: Velocity profiles of Couette flow (circles) and Poiseuille-like flow with body force $f=0.008 \sigma / \tau^{2}$ (squares) at $\epsilon_{\mathrm{s}}=0.6 \epsilon$ are shown. Smooth thick solid lines are the fits to the velocity profiles, measured away from the substrate. The positions $Z_{C}$ and $Z_{P}$, where extrapolated macroscopic profiles reach the velocity of the wall, $v_{\text {wall }}^{C}=-0.075 \sigma / \tau$ and $v_{x}^{\mathrm{P}}\left(-\mathrm{Z}_{\mathrm{P}}\right)=0$ for Couette and Poiseuille flows, respectively, are indicated. These noslip positions depend on the type of the flow and therefore cannot be used as a boundary condition.

Eqs. (3.38) and (3.39), we relate the extrapolated positions $Z_{C}$ and $Z_{P}$, which we extract from the simulations, to the slip length, $\delta=\sqrt{Z_{C}^{2}-Z_{P}^{2}}$, and boundary position, $z_{\mathrm{h}}=\mathrm{Z}_{\mathrm{C}}+\delta[106,117]$.

To generate Poiseuille flow, we impose body forces $f$ of strength $0.001-0.010 \sigma / \tau^{2}$ (cf. Fig. 3.17b). An external force injects energy into the system, which is removed by the thermostat, keeping the temperature constant. In order to preserve a linear response of the liquid, only the smallest force was applied at small values of $\epsilon_{\mathrm{S}}$, whereas for $\epsilon_{\mathrm{s}} \geqslant 0.6 \epsilon$ all four forces were used.

The positions of an effective no-slip plane, where the extrapolation of the hydrodynamic velocity field vanishes $-Z_{C}$ for Couette flow and $Z_{P}$ for Poiseuille flow - are marked in velocity profiles of the liquid confined between two apposing substrates with strength of solid-liquid interaction $\epsilon_{\mathrm{s}}=0.6 \epsilon$ in Fig. 3.18. These positions depend on the type of the flow and, hence, cannot be used to parameterize a boundary condition. Instead, the distortion of the microscopic velocity profile can be described by the hydrodynamic boundary position $z_{\mathrm{h}}$, where Navier's condition 3.36 is applied (vertical dashed line) and slip length $\delta$.

\subsubsection{Hydrodynamic boundary position and slip length}

Using the simulation data obtained from Couette and Poiseuille flows, we can independently determine the slip length, $\delta$, and the position of the hydrodynamic boundary, $z_{\mathrm{h}}$. In Fig. 3.19 we present the distance between the boundary position $z_{\mathrm{h}}$ and the position, 


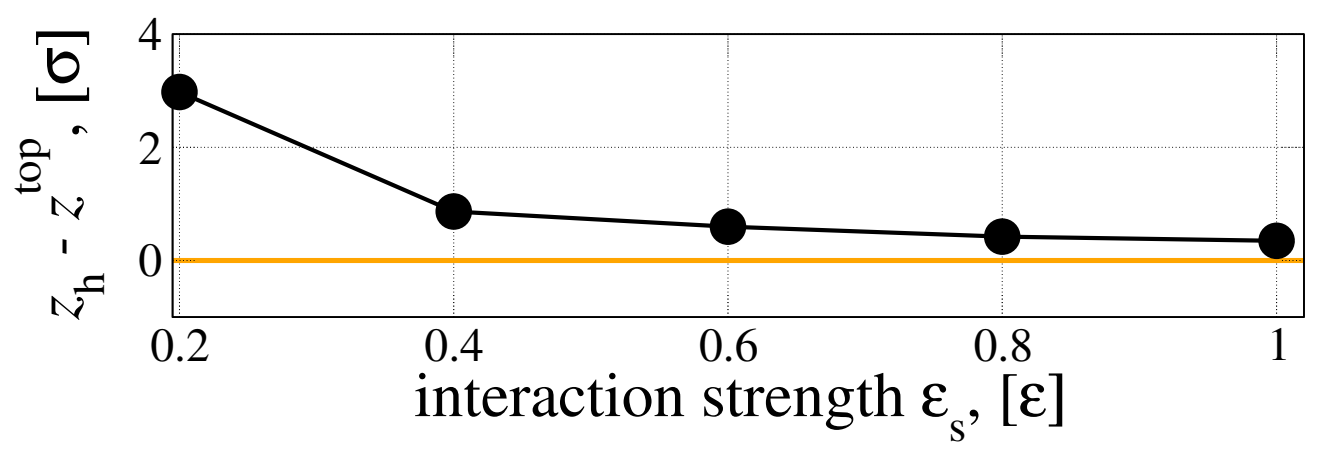

Figure 3.19: Distance of the position, $z_{\mathrm{h}}$, at which Navier's boundary condition 3.36 is to be applied, to the innermost atomic layer of the substrate $z^{\text {top }}$ for channels with flat walls. Positive values, $z_{\mathrm{h}}-z^{\text {top }}>0$, indicate that the effective boundary position is closer to the center of the channel than any particle of the wall.

$z^{\text {top }}$, of the innermost atomic layer of the substrate (this position is therefore the origin indicated by thick horizontal line in Fig. 3.19). It is interesting to notice, that even in the case of flat walls, at small $\epsilon_{\mathrm{s}}$ the hydrodynamic boundary is found somewhat inside the liquid, but not directly at the solid-liquid interface. However, the more attractive becomes the solid, the closer is $z_{\mathrm{h}}$ to the interface.

The second parameter of the hydrodynamic boundary condition is the slip length, $\delta$, or, equivalently, the friction coefficient $\lambda=\eta / \delta$. If the macroscopic continuum description of the velocity profile were accurate up to the position of the hydrodynamic boundary, $z_{\mathrm{h}}$, and the liquid properties did not change in the vicinity of the substrate, then we could interpret the friction coefficient as the proportionality coefficient between the microscopic friction stress and the local velocity of the fluid flowing past the substrate. In this case, one can compute the friction coefficient via a Green-Kubo relation for the autocorrelation function of the tangential force, $F_{x}$, between the liquid and the substrate [105],

$$
\frac{\eta}{\delta}=\lambda=\frac{1}{k_{\mathrm{B}} T A} \int_{0}^{+\infty} \mathrm{d} t\left\langle F_{x}(t) F_{x}(0)\right\rangle
$$

where $A=L_{x} L_{y}$ is the area of the substrate. The analysis of Barrat and Bocquet [105] suggests that $\lambda$ be proportional to the square of the attraction between liquid and substrate, $\lambda \sim \epsilon_{\mathrm{s}}^{2}$. Previous simulations for a Lennard-Jones polymer liquid on a LennardJones solid corroborated this relation [34], and we also observe this dependence on $\epsilon_{\mathrm{S}}$ for our flat substrate as demonstrated by the dashed line in Fig. 3.20.

Utilizing both surface parameters, the position of the hydrodynamic boundary $z_{\mathrm{h}}$ and the slip length $\delta$, we conclude that in the vicinity and above wetting transition $\left(\epsilon_{\mathrm{s}} \geqslant\right.$ $0.8 \epsilon$ our method is consistent with a no-slip boundary, as in macroscopic case. Indeed, the hydrodynamic boundary in this case is situated at the solid-liquid interface (cf. Fig. 3.19) and the slip length $\delta \rightarrow 0$ (cf. Fig. 3.20). However, a finite slippage is present 


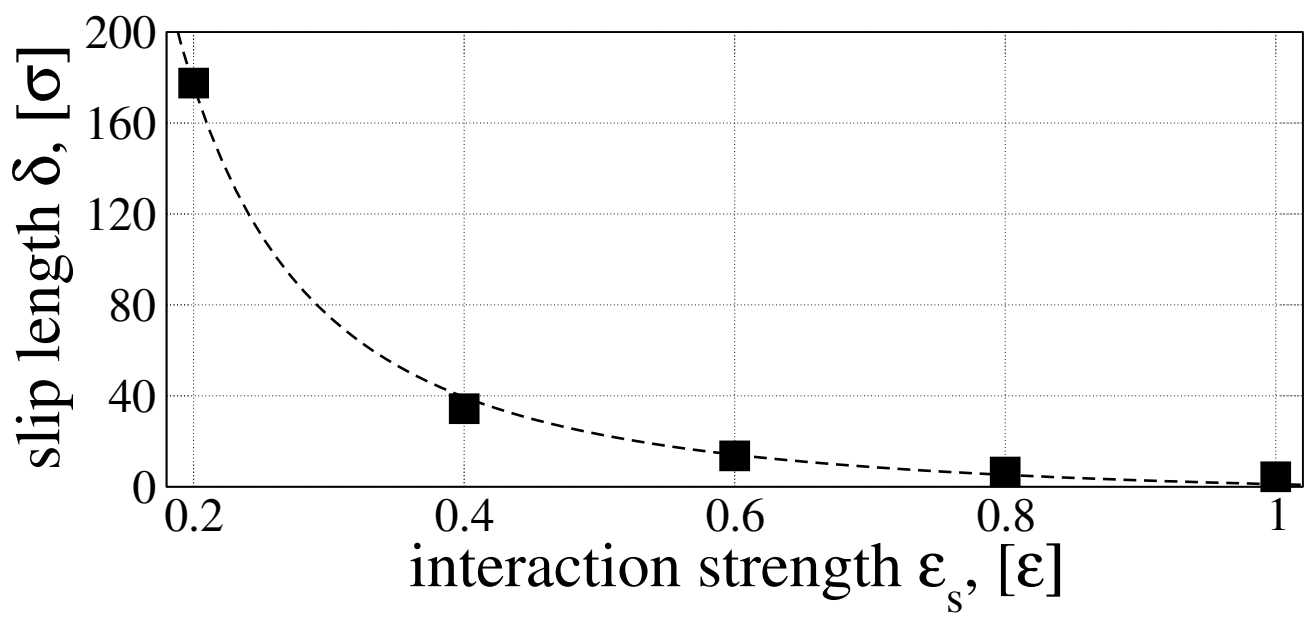

Figure 3.20: The slip length $\delta$ for a liquid confined between flat substrates. The dashed line is the analytical prediction of Barrat and Bocquet [105] based on Green-Kubo relation for friction coefficient.

at less attractive substrates and in the case of a very weak attraction, $\epsilon_{\mathrm{s}}=0.2 \epsilon$, a very thin vapor layer is formed at the solid that act as a perfect lubricant. The slippage becomes enormous and exceeds the linear dimensions of the simulation domain, and the hydrodynamic boundary position $z_{\mathrm{h}}$ is found inside the liquid phase at the distance of 3 molecular diameters from the solid-liquid boundary (cf. Fig. 3.19).

\subsection{Discussion}

The equilibrium properties of polymer droplets have been studied by Molecular Dynamics simulation of a coarse-grained particle-based model and a continuum description in terms of an effective interface Hamiltonian. We have devised a simple method to compute the interface potential for laterally corrugated substrates, which is based on the anisotropy of the pressure inside the film. This general computational strategy can be applied to dense liquids of large macromolecules and can be implemented in standard Molecular Dynamics programs. Using the so-determined interfacial tensions and the interface potential in the continuum model, we find quantitative agreement between both descriptions if (i) the full curvature is used in the continuum model for large contact angles and (ii) the size of the drop is larger than the lateral correlation length, $\xi_{\|}$, of interface fluctuations. We also find that for contact angles up to about 30 degree the long-wavelength approximation that is normally used in thin film models describes the droplet shapes even quantitatively quite well.

These results demonstrate that the tensions and the interface potential capture the relevant information that needs to be passed on to a continuum model to describe the equilibrium shape of droplets, including the deviations from the spherical cap shape in the vicinity of the three-phase contact line. This is an excellent starting point for com- 
paring the dynamics of droplets driven by external forces, which we will pursue in the future.

Additionally, we considered dynamical properties of liquid flows confined between flat substrates. It was shown, that applying macroscopic Navier's condition to a microscopic model, two different setups of the flow should be considered: Couette and Poiseuille flows. Then, the solid-liquid interface is described by two material parameters: slip length, $\delta$, and hydrodynamic boundary position, $z_{\mathrm{h}}$. The latter does not necessarily corresponds to the position of the solid-liquid interface, but even in the case of flat walls it is located somewhat inside the liquid. 


\section{CHAPTER 4}

\section{SYMMETRICALLY STRUCTURED SUBSTRATES}

Some thoughts have glue on them. Smilla

Peter Høeg

This chapter considers droplets on and flows past symmetrically structured substrates. In contrast to flat substrates, the surface corrugated by regular rectangular grooves allows for a rich liquid behavior. Macroscopically, a liquid on this structured substrate may either fill the cavities between corrugations (Wenzel state [118]) or build a straight liquid-vapor interface on the top of the corrugations (Cassie state [119] or fakir state). Microscopically, we show that a broad crossover regime exists between these two states.

At first, we present the substrate of $X Y z$ unit cells that was initially introduced in Sec. 2.1. The difference between flat substrates composed of $X Y Z$ and $X Y Z$ unit cells is considered in the beginning of Sec. 4.1. Later in this section, we quantify the difference in mobility of droplets in Cassie and Wenzel states. To this end, a technique of free-energy barrier estimation for a drop moving across the corrugated substrate is proposed. Employing this method, we show that, as expected, the height of the energetic barrier for a drop in the Cassie state is significantly reduced in comparison to the one in the Wenzel state.

We proceed with the study of the dynamics of droplets pushed over superhydrophobic and hydrophobic surfaces. It was previously shown that at small and moderate equilibrium contact angles, the velocity profiles taken from the solid-liquid interface to the apex of the drop, are of the parabolic form [27]. On the other hand, mesoscopic lattice Boltzmann simulations showed that for droplets with high contact angles (ca. 145 ${ }^{\circ}$ ) the velocity profiles start as parabolic at the substrate, but very fast reach a linear form, indicating rolling [72]. We investigate if the contact angle is the only parameter, controlling this behavior. Additionally, the influence of the pinning of three-phase contact lines is inspected. 


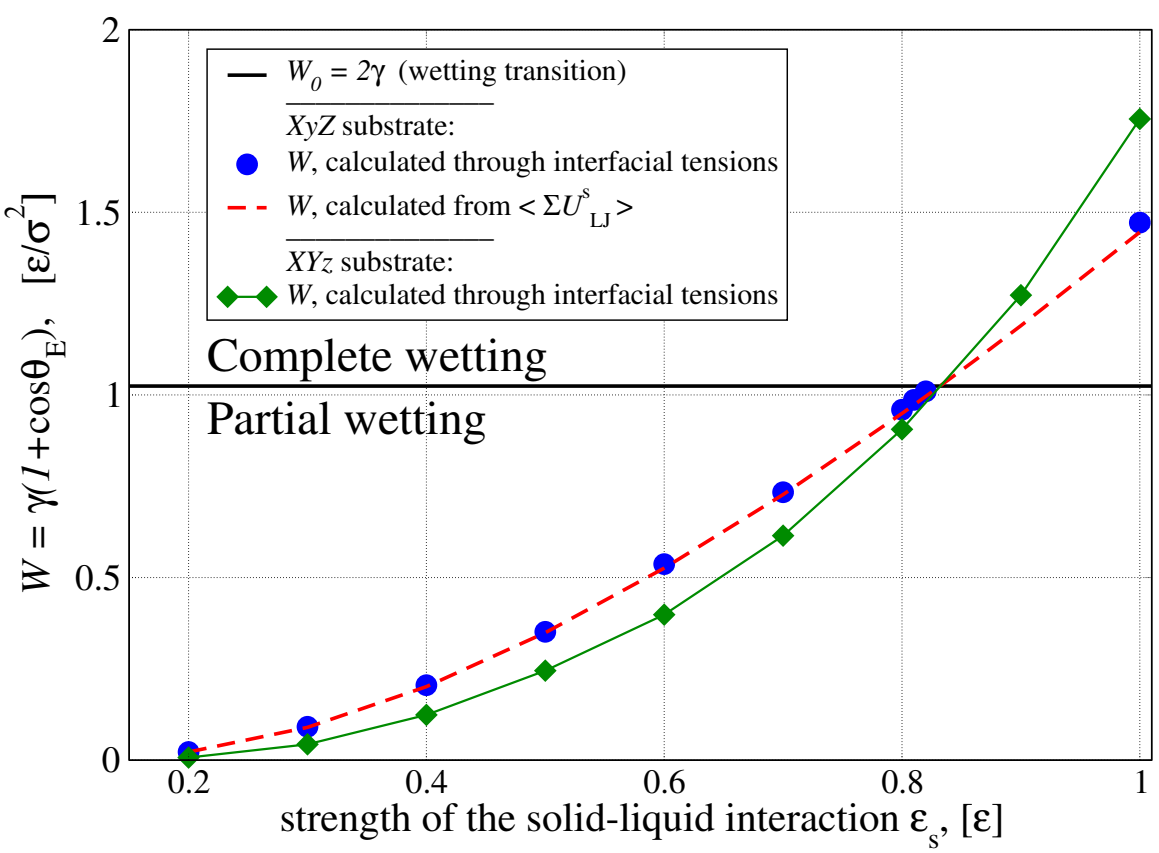

Figure 4.1: Adhesion energy per unit area of substrates composed of $X Y Z$ (circles) and $X Y Z$ (diamonds) unit cells according to Eq. 3.15. For the XyZ unit cell, the integration method is shown by the dashed line (cf. Eq. 3.20). The thick solid horizontal line marks the adhesion energy $W_{0}=2 \gamma$. The wetting transition for both substrates happens at the same strength of solid-liquid interaction $\epsilon_{\mathrm{s}}^{\mathrm{wet}} \approx 0.83 \epsilon$.

In the last section we study the influence of corrugation, wettability and pressure on slippage and friction at the solid-liquid interface. For one-dimensional, rectangular grooves, we observe a gradual crossover between the Wenzel and the Cassie states. In accord with previous Chapter, employing two independent flow set-ups, we characterize the near-surface flow by the slip length, $\delta$, and the position, $z_{\mathrm{h}}$, at which viscous and frictional stresses are balanced according to Navier's partial slip boundary condition. This hydrodynamic boundary position depends on the pressure inside the channel and may be located above the corrugated surface. In the Cassie state, we observe that the edges of the corrugation contribute to the friction.

\subsection{Static and dynamic properties of polymer droplets}

The substrate model in this and the following Chapters is somewhat different from the one being in use before. As mentioned in Sec. 2.1, we change the orientation of the unit cell from $X y Z$ to $X Y Z$. The character of transformation is shown in Fig. 2.1. This kind of change allows one to create posts with squared cross-section, as the lengths of lattice vectors $\vec{x}$ and $\vec{y}$ are equal. However, in the end this opportunity has not been 


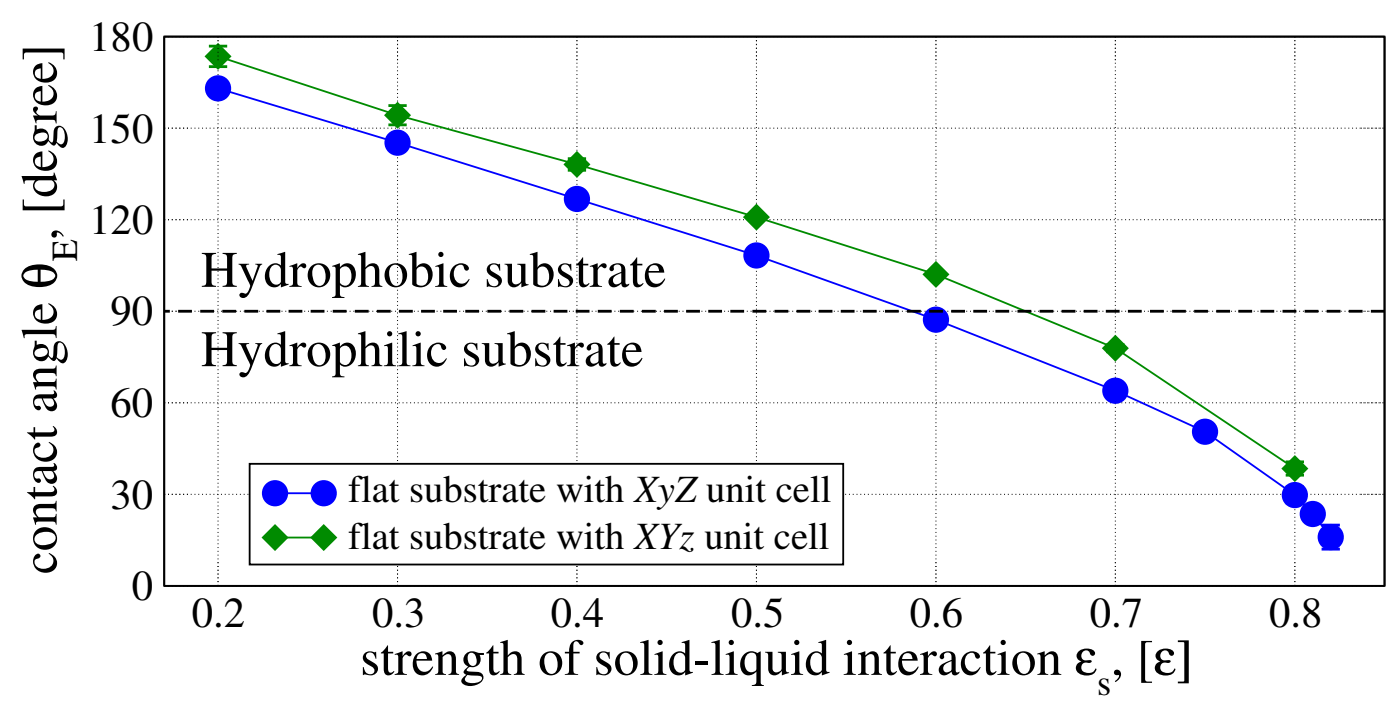

Figure 4.2: Equilibrium contact angles of the droplets, $\theta_{\mathrm{E}}$, as a function of the solid-liquid interaction strength $\epsilon_{\mathrm{s}}$ for the substrates composed of $X Y Z$ (circles) and $X Y z$ (diamonds) unit cells according to Young-Laplace law (Eq. 3.14). For XYZ unit cell, the solid-vapor surface tension was considered to be $\gamma_{S V}=0$. Solid lines are guides for the eyes. Horizontal dashed line separates hydrophobic $\left(\theta_{\mathrm{E}}>90^{\circ}\right)$ from hydrophilic $\left(\theta_{\mathrm{E}}<90^{\circ}\right)$ regions.

exploited ${ }^{1}$, because the behavior of the liquid on quasi-one-dimensional grooves appeared to be complicated enough. Nevertheless, the study of liquids on substrates with posts gives additional insights into wetting phenomena [120-122] and we are planning to elaborate on this in future.

Since the model of the substrate was changed, several quantities have been recalculated. All the properties of the liquid itself (e.g. its bulk density $\rho_{\text {coex }}$, coexistence pressure $p_{\text {coex }}$, viscosity $\eta$ and liquid-vapor interfacial tension $\gamma$ ) remain unchanged. Substrate composition affects only quantities related to solid-liquid interaction. Employing the methods presented in Sec. 3.1, we determine adhesion energy and equilibrium contact angles of liquid at new flat substrate. The comparison of adhesion energies (Eq. 3.15) of flat substrates composed of $X Y Z$ and $X Y Z$ unit cells is shown in Fig. 4.1. The wetting transition is located at approximately the same strength of solidliquid interaction, $\epsilon_{\mathrm{s}}^{\mathrm{wet}} \approx 0.83 \epsilon$, what we consider rather like a coincidence than being originated by some physical reason.

The better understanding of the difference between two flat substrates gives a direct comparison of equilibrium contact angles of droplets, presented in Fig. 4.2. At fixed strength of solid-liquid interaction $\epsilon_{\mathrm{s}}$ the $X Y z$ substrate (diamonds) provides higher contact angles than $X y Z$ one (circles). It is due to the fact that the length of lattice vector $\vec{y}$ is increased and the attraction to the $X Y z$ solid is somewhat smaller than to the $X y Z$ one. The solid may be either hydrophobic $\left(\theta_{\mathrm{E}}>90^{\circ}\right)$ or hydrophilic $\left(\theta_{\mathrm{E}}<90^{\circ}\right)$. The

\footnotetext{
${ }^{1}$ Later, in Sec. 4.1.3, one of the substrate we study is made of posts arranged in chessboard order. However, their cross-section is rectangular.
} 


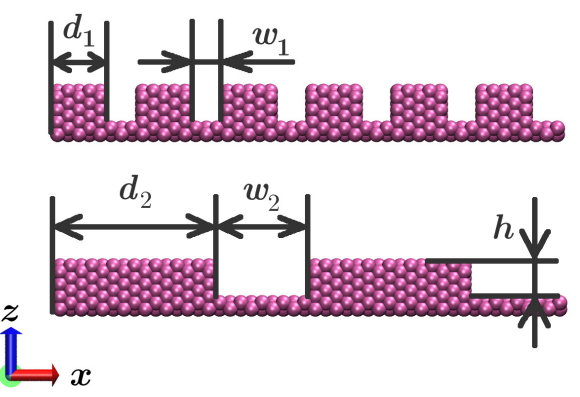

Figure 4.3: The geometries of the structured substrates. Patterning is present only in $x$ direction.

horizontal dashed line separates these two regions. Since we will focus on hydrophobic substrates, the non-zero values of solid-vapor surface tension $\gamma_{S V}$ in the vicinity of the wetting transition were not taken into account.

The next section introduces the macroscopic states of the droplet placed on the top of a structured substrate.

\subsubsection{Cassie and Wenzel states}

We consider a regularly corrugated surface topography as sketched in Fig. 4.3. The structured substrates are composed of $X Y z$ unit cells. The macroscopic contact angle that a liquid drop makes with the solid substrate is dictated by the balance of surface tensions according to Young's equation 3.14. Surface roughness tends to amplify the wetting behavior, i.e., structuring a hydrophobic substrate (contact angle of a drop greater than $90^{\circ}$ ) by grooves, one increases the contact angle and may render the substrate superhydrophobic $[118,119,123,124]$.

$$
\cos \theta_{\mathrm{C}}=\varphi \cos \theta_{\mathrm{E}}+\varphi-1,
$$

where $\theta_{\mathrm{C}}$ is the contact angle in the Cassie state, and $\theta_{\mathrm{E}}$ is the contact angle on a flat substrate.

When the liquid does not fill the cavities (Cassie state as shown in Fig. 4.4a), the contact angle changes according to Cassie's formula [119] In the Wenzel state shown in Fig. 4.4b, the liquid wets the substrate completely and the contact angle is described by Wenzel's formula [118]

$$
\cos \theta_{\mathrm{W}}=r \cos \theta_{\mathrm{E}}
$$

where $\theta_{\mathrm{W}}$ is the contact angle in the Wenzel state, and $r$ stands for roughness of the substrate. The parameter $r$ is the ratio of the actual substrate area wetted by the liquid to the projected area of the substrate. $r \geq 1$ and $r=1$ for a flat unstructured substrate. Our model of the substrate corresponds to the roughness $r=\frac{d+w+2 h}{d+w}$, where $w$ is the 


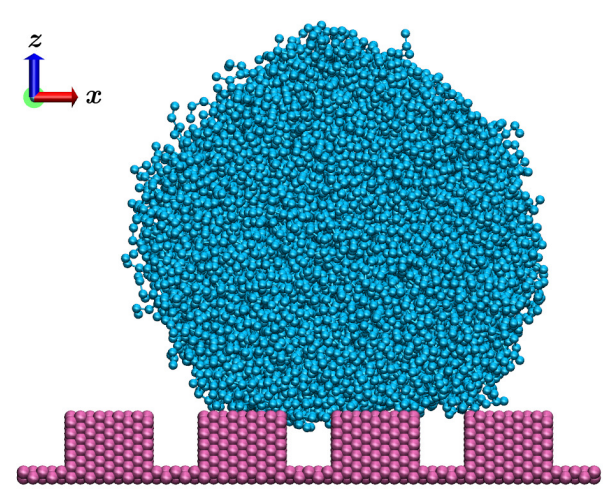

(a)

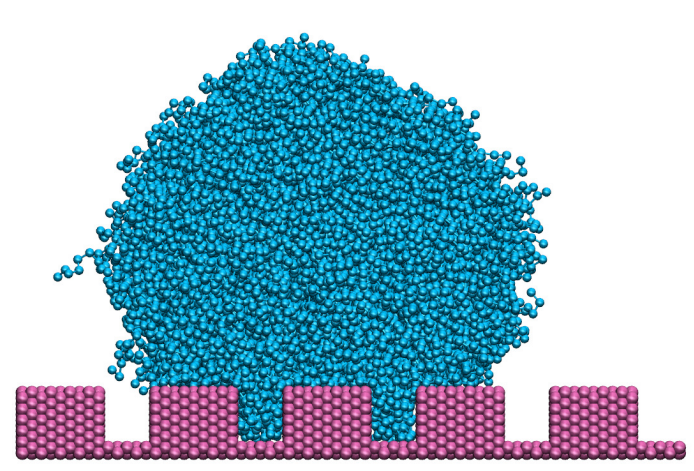

(b)

Figure 4.4: (a) A droplet in Cassie state does not fill the grooves of the substrate. (b) A droplet in Wenzel state fills the substrate cavities, creating solid-liquid interfaces at the bottom and walls of the groove. The strengths of solid-liquid interaction are $0.3 \epsilon$ and $0.5 \epsilon$ for (a) and (b), respectively.

width of the groove. For the finely and coarsely corrugated substrates the roughness is given by $r_{1}=1.865$ and $r_{2}=1.289$, respectively. The values of the contact angles for a flat surface were calculated in Sec. 4.1 and the predictions of Cassie and Wenzel formulae (with roughnesses $r_{1}$ and $r_{2}$ ) are compiled in Table 4.1.

We emphasize that Cassie and Wenzel predictions, given by Eqs. (4.1) and (4.2), refer to macroscopic amounts of liquid and do not account for thermal fluctuations of the liquid-vapor interface spanning the grooves or the influence of the three-phase contact line at the edges of the grooves. Since the pinning of a liquid on a patterned substrate occurs in MD simulations, there is a hysteresis of the values of the contact angles. Thus, if one simulates a droplet, the estimates of the contact angle will not necessarily correspond to the Cassie or Wenzel values.

In the following section we quantify the difference in mobility of droplets at various solid-liquid interaction strength $\epsilon_{\mathrm{s}}$ on the finely corrugated substrate $r_{1}$.

\begin{tabular}{l|c|c|c|c}
$\epsilon_{\mathrm{S}}$ & $\theta_{\mathrm{E}},\left[^{\circ}\right]$ & $\theta_{\mathrm{C}},\left[^{\circ}\right]$ & $\left.\theta_{\mathrm{W}}\right|_{r=r_{1}},\left[^{\circ}\right]$ & $\left.\theta_{\mathrm{W}}\right|_{r=r_{2}},\left[^{\circ}\right]$ \\
\hline $0.2 \epsilon$ & 173.5 & 174.9 & - & - \\
$0.3 \epsilon$ & 154.2 & 159.7 & - & - \\
$0.4 \epsilon$ & 138.1 & 147.2 & - & 163.5 \\
$0.5 \epsilon$ & 120.8 & 134.0 & 162.8 & 131.3 \\
$0.6 \epsilon$ & 102.1 & 120.4 & 113.0 & 105.7
\end{tabular}

Table 4.1: Contact angles, $\theta_{\mathrm{E}}, \theta_{\mathrm{C}}$, and $\theta_{\mathrm{W}}$, on flat and structured substrates when the liquid is in the Cassie or the Wenzel states, respectively. Data are obtained from the measured values of the surface and interfacial tensions of the planar solid-liquid and liquid-vapor tension using Eqs. (4.1) and (4.2) for the two different corrugations $r_{1}$ and $r_{2}$. 


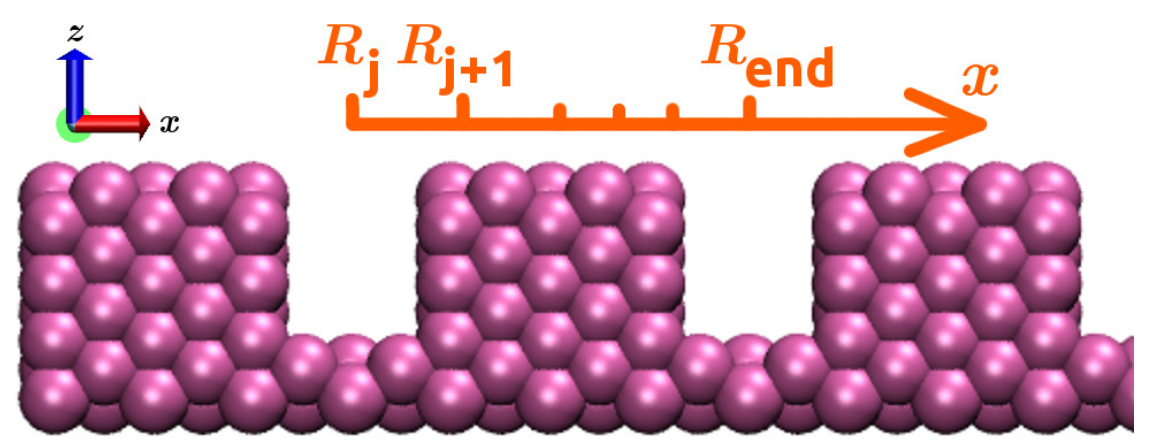

Figure 4.5: Integration path to calculate the energetic barrier. $\left\{R_{j}, R_{j+1}, \ldots, R_{\text {end }}\right\}$ denote reaction coordinates at which the $\mathrm{CM}$ of the droplet is constrained in $x$-direction. The path starts in the middle of one groove and ends in the middle of the next one.

\subsubsection{Energetic barrier and constraint force}

The attraction strength $\epsilon_{\mathrm{s}}$ determines the mobility of the droplet. While at small interaction strengths the droplet is free to diffuse on the top of the corrugations, at high attraction it is immobile and remains on the same grooves for a long time. To quantify this difference we investigate the height of the energetic barrier for the drop, when its center of mass $(\mathrm{CM})$ is moving across the finely corrugated substrate $r_{1}$.

The difference in free energy, $\Delta F_{j}^{j+1}$, between two CM positions along $x$-axis, $R_{j}$ and $R_{m}$, can be formulated by

$$
\Delta F_{j}^{m}=\int_{R_{j}}^{R_{m}} \frac{\partial F}{\partial R} d R
$$

Now, suppose we fix the $\mathrm{CM}$ at the position $R=\frac{1}{N} \sum_{i} r_{i}$, where $N$ is the number of molecules and $r_{i}$ is the coordinates of $i$-th bead. The free energy is then defined as $F=-k_{\mathrm{B}} T \ln (Q(R) K(P))$, where $Q(R)$ and $K(P)$ are the configurational and kinetic part of the constraint partition function, respectively. Then,

$$
\frac{\partial F}{\partial R}=-\frac{1}{\beta} \frac{\partial \ln (Q(R) K(P))}{\partial R}=-\frac{1}{\beta} \frac{1}{Q(R)} \frac{\partial Q(R)}{\partial R} .
$$

Let us define the configurational part explicitly as

$$
Q(R)=\int d^{N} r_{i} \exp \left(-\beta U\left[r_{i}\right]\right) \delta\left(R-\frac{1}{N} \sum_{i} r_{i}\right)
$$

where $\delta(\ldots)$ is the delta function, and introduce relative coordinates $\tau_{i}=r_{i}-R$. Showing that the Jacobian $\left|\partial\left(\tau_{i}\right) / \partial\left(r_{i}\right)\right|=1$ for a fixed value of $R$, we can rewrite Eq. 4.5 as

$$
Q(R)=\int d^{N} \tau_{i} \exp \left(-\beta U\left[\tau_{i}+R\right]\right) N \delta\left(\sum_{i} \tau_{i}\right)
$$

If one now differentiates $Q(R)$ with respect to $R$,

$$
\frac{\partial Q(R)}{\partial R}=\int d^{N} \tau_{i} \exp \left(-\beta U\left[\tau_{i}+R\right]\right) N \delta\left(\sum_{i} \tau_{i}\right) \cdot \sum_{i}-\beta \frac{\partial U}{\partial \tau_{i}},
$$




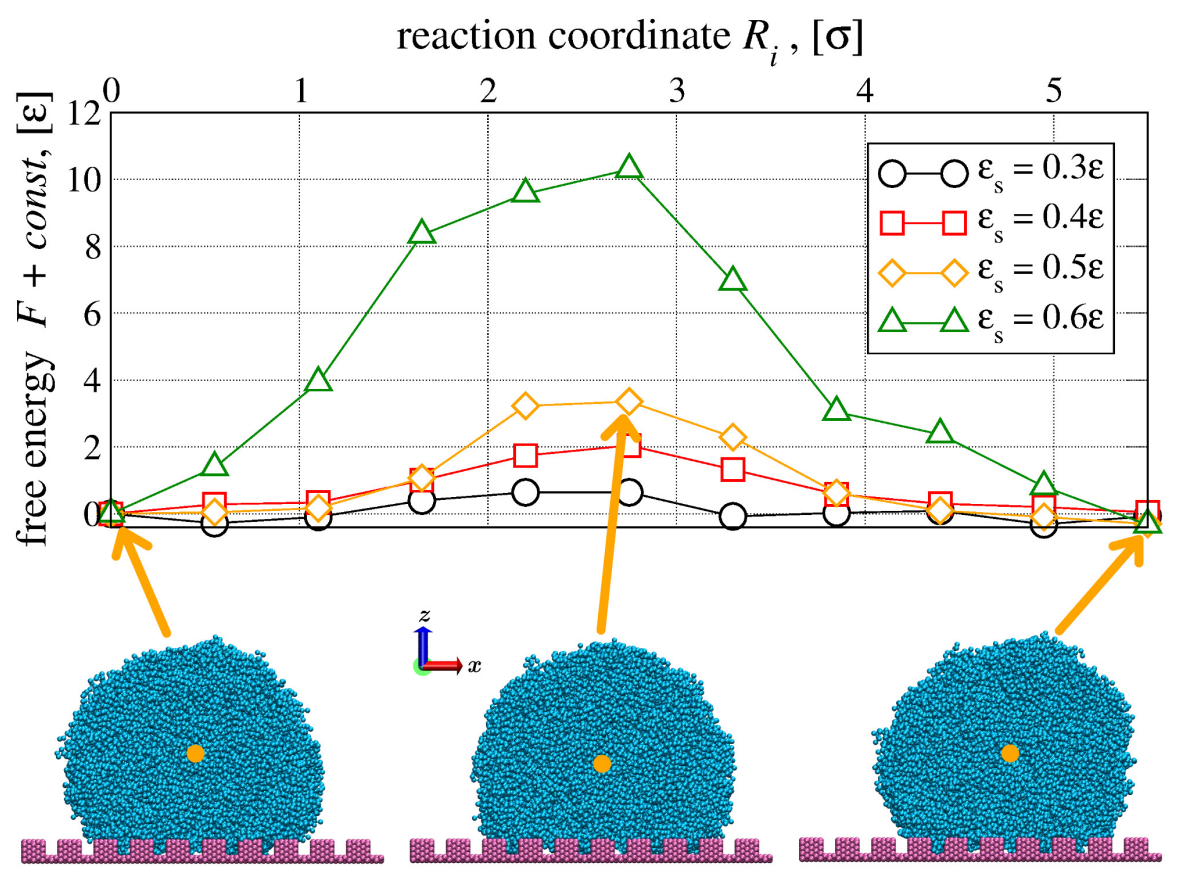

Figure 4.6: Energetic barriers for droplets of ca. $N=20000$ beads, when moving its $\mathrm{CM}$ from one cavity to the neighboring one. Different symbols stand for various attraction strength $\epsilon_{\mathrm{S}}$ of the finely corrugated substrate $r_{1}$. Arrows indicate values of reaction coordinate, $R_{j}$, at which snapshots are taken for the droplet at $0.5 \epsilon$. Positions of the CM of droplets are indicated by circles on the snapshots.

one obtains from Eq. 4.4

$$
\begin{aligned}
\frac{\partial F}{\partial R} & =-\frac{1}{\beta} \frac{1}{Q(R)} \int d^{N} \tau_{i} \exp \left(-\beta U\left[\tau_{i}+R\right]\right) N \delta\left(\sum_{i} \tau_{i}\right) \cdot \sum_{i}-\beta \frac{\partial U}{\partial \tau_{i}} \\
& =\left\langle\sum_{i} \frac{\partial U}{\partial \tau_{i}}\right\rangle=\left\langle\sum_{i} \frac{\partial U}{\partial r_{i}}\right\rangle=-\left\langle F_{\mathrm{CM}}\right\rangle,
\end{aligned}
$$

where angular brackets stand for ensemble averages and $F_{\mathrm{CM}}$ is the force onto the center of mass of the droplet to keep it at the fixed position $R$. To calculate the energetic barrier for a droplet when moving across the corrugations, we constrain its $\mathrm{CM}$ at positions $\left\{R_{j}, R_{j+1}, \ldots, R_{\text {end }}\right\}$ and measure the average constrain force onto the $\mathrm{CM},\left\langle F_{\mathrm{CM}}\right\rangle$, at these positions. Then, according to Eq. 4.3, we integrate these forces along the reaction coordinate $R$ and obtain the differences in free energy between two neighboring CM positions upto some constant. The integration path is shown in Fig. 4.5.

The droplets of ca. $N=20000$ monomers are considered and the strength of solidliquid interaction $\epsilon_{\mathrm{S}}$ is varied in hydrophobic range from $0.2 \epsilon$ to $0.6 \epsilon$. The energetic barriers for these systems are presented in Fig. 4.6.

The first notable feature of the plot is that the values of free energy at the beginning of the paths and at the end of them are equal. This proves the reversibility of the free energy calculation. Moreover, the energetic barriers are symmetric, as the topography of the substrate implies. Indeed, one can start at the coordinate $R_{\text {end }}$ and take the 
integration path into opposite direction. Second, we note that the heights of the barriers are increasing with strength of solid-liquid interaction $\epsilon_{\mathrm{S}}$, as it was initially expected. At last, to demonstrate the most undesirable position of the droplet's CM, we show snapshots from simulations at attraction strength $0.5 \epsilon$, when the barrier is the highest and the lowest (the correspondence to the reaction coordinate $R$ is shown by arrows). The positions of the $\mathrm{CM}$ are indicated by the circle on the snapshots.

By implementing constraints in the simulations, we calculated the free energy barrier when moving the $\mathrm{CM}$ of the drop by one period of the substrate. While at small interaction strengths $\epsilon_{\mathrm{s}}$ the droplet may easily overcome the energetic barrier, at high values of $\epsilon_{\mathrm{s}}$ it is highly unprobable to happen, as the barrier is significantly higher. This indicates that in order to make such droplets move one has to apply an external force. The dynamics of droplets pushed over hydrophobic substrates will be studied in thext section.

\subsubsection{Velocity profiles of droplets moving under a body force}

In the limit of small Reynolds numbers, the Navier-Stokes equation takes the so-called Stokesian form [101]

$$
0=-\nabla p+\eta \Delta \vec{u}+\vec{f}
$$

where $\vec{u}$ is three-dimensional velocity field in the droplet, $\vec{f}$ is the body force acting on all its particles and $\nabla p$ is the pressure gradient.

In the lubrication approximation (small equilibrium contact angles) one expects that the velocity profile in $x$-direction, $v_{x}$, is a parabolic function of the distance to the surface, $z$ [89]. Indeed, in this case Stokes equation can be of a particularly simple form

$$
\frac{\partial^{2} v_{x}}{\partial z^{2}}=-\frac{\rho_{\text {coex }} f}{\eta}
$$

and it was shown that the parabolic velocity profile is preserved even for contact angles upto $130^{\circ}$ on flat substrates [27]. However, by scaling arguments, for droplets of very large contact angles the profile is linear [125] and a rolling motion is observed. The crossover between these two regimes was found in mesoscopic lattice Boltzmann simulations [72]. There, on both, hydrophobic and superhydrophobic substrates covered by posts, the velocity profile is quadratic in $z$ in the vicinity of the substrate, but reaches the linear form further away from it.

In our simulations, we investigate the influence of the corrugation onto velocity profiles of droplets. The drops of equilibrium contact angles $120.8^{\circ} \leqslant \theta_{\mathrm{E}} \leqslant 154.2^{\circ}$ are considered, providing macroscopic contact angles on corrugated substrates in the range of $131.3^{\circ} \leqslant \theta_{\mathrm{E}} \leqslant 159.7^{\circ}$ (cf. Table 4.1). At high contact angles, $\theta_{\mathrm{C}} \approx 145^{\circ}$, we expect a combination of parabolic and linear profiles as was observed by lattice Boltzmann 
simulations. However, at very high contact angles, the profile may be of the linear form, indicating pure rolling motion. At smaller contact angles, $\theta_{\mathrm{C}}<145^{\circ}$, it is not obvious how the velocity depends on the distance to the substrate, $z$. If the corrugation influences the dynamics, deviations from a purely quadratic profile, measured for our liquid model in Ref. [27], may be observed.

In this section we model droplets of $N=200000$ monomers, as they are big enough to justify the behavior of the velocity profile inside. The strength of the solid-liquid interaction varies from $0.3 \epsilon$ to $0.5 \epsilon$. The length of the spanned dimension is $L_{y} \approx 30 \sigma$, and the droplets are cylindrical. We use the finely corrugated substrate $r_{1}$, presented in Sec. 4.1.1. Keeping the ratio of the solid covered by ridges to the projected area constant, $\varphi=0.625$, we also use the substrate composed of the posts arranged in chessboard order. It was suggested, that such topography reduces the pinning at the three-phase contact lines, allowing the higher droplet's mobility [126]. This substrate is referred to as the one with $r_{1}^{\text {mod }}$ corrugation to indicate that it has a tight relation to $r_{1}$, but is modified.

At the smallest attraction to the substrate, $\epsilon_{\mathrm{s}}=0.3 \epsilon$, the body force is taken to be $f=0.00001 \sigma / \tau^{2}$. It is big enough to provide the motion of the droplet [127], but is still small to prevent it from detaching. At $0.4 \epsilon$ and $0.5 \epsilon$ a somewhat higher force is used due to the same reasons, $f=0.000025 \sigma / \tau^{2}$.

The velocity profiles inside the drops are taken not only in a vertical slab, where the $\mathrm{CM}$ is located, but also at the lateral distance $10 \sigma$ in front and behind it (relating to the direction of the body force). However, for a better comprehension, only velocities $v_{x}(z)$ in the slab with the CM are plotted in Fig. 4.7.

At first, we discuss the character of the dependence of the velocity $v_{x}$ on coordinate $z$. At small attraction strength, $\epsilon_{\mathrm{s}}=0.3 \epsilon$, the linear approximations in the region $40 \sigma \leqslant$ $z \leqslant 90 \sigma$ provide a good agreement with the simulation data even in the vicinity of the substrate. Hence, we conclude that at this regime the droplet's velocity profile is of the linear form across its entire height, $z$, and the drop rolls over the substrate. At higher attraction strengths, the velocity profiles at the substrate deviate from the linear behavior. It indicates, that the character of the dependence changes to the parabolic one. Unfortunately, the statistics for highest solid-liquid interaction strength $\epsilon_{\mathrm{s}}=0.5 \epsilon$ is not good enough to see this distinctly, but at $\epsilon_{\mathrm{s}}=0.4 \epsilon$ this effect is more pronounced (cf. circles and correspondent linear fits in inset of Fig. 4.7).

Now, we turn our attention to the comparison of two substrates: with $r_{1}$ and $r_{1}^{\text {mod }}$ corrugations (open vs. shaded symbols in Fig. 4.7). It is interesting to notice, that although at solid-liquid interaction strengths $0.3 \epsilon$ and $0.5 \epsilon$ the droplet has a somewhat higher velocity on chessboard substrate than on the one with grooves, this effect vanishes at intermediate strength of interaction, $0.4 \epsilon$. Since the sizes of the droplets are equal, the 


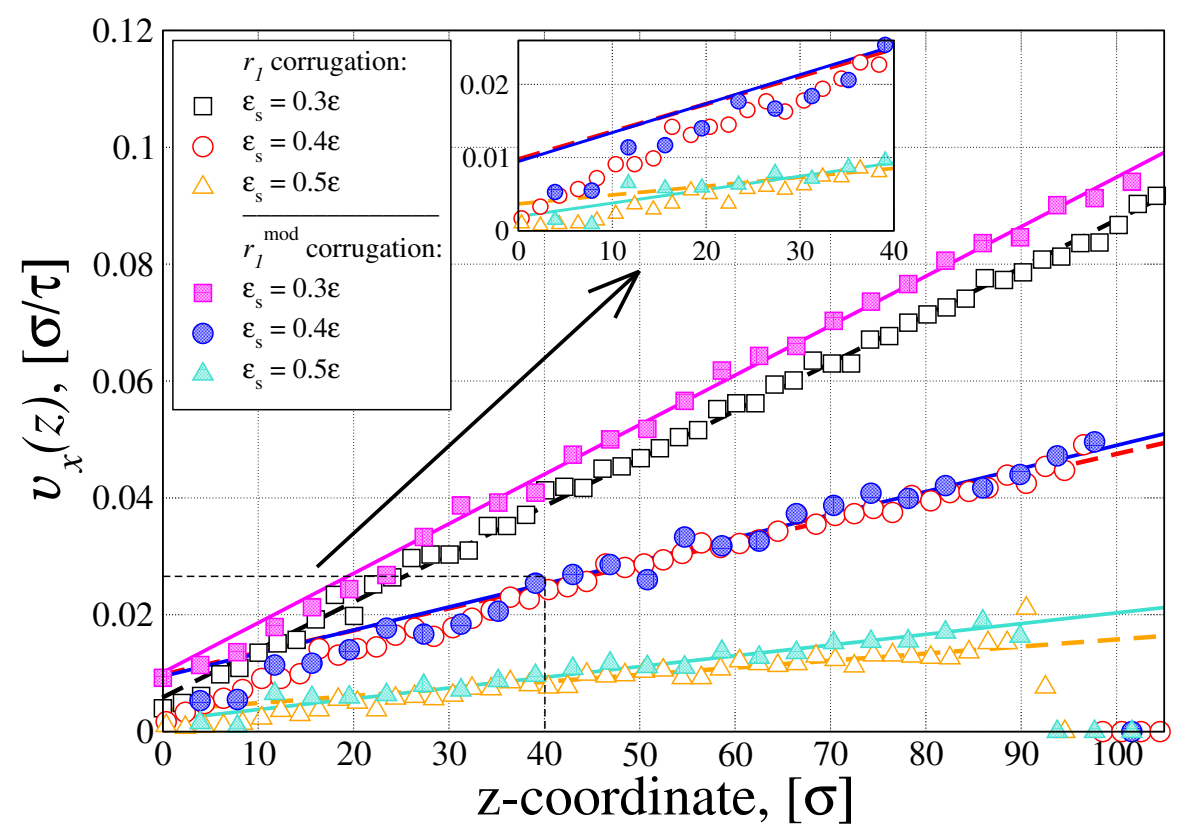

Figure 4.7: Velocity profiles of droplets as a function of the distance to the substrate, $v_{x}(z)$. The open symbols stand for corrugation $r_{1}$, shaded ones - for corrugation $r_{1}^{\bmod }$. Squares, circles and triangles correspond to the solid-liquid interaction strengths $0.3 \epsilon, 0.4 \epsilon$ and $0.5 \epsilon$, respectively. Lines are linear approximations to the data in the region away from the substrate, $(40-90) \sigma$. The inset zooms into the region $z<40 \sigma$ that does not affect linear fits.

only possible explanation of this behavior lies in non-trivial changes of an effective friction coefficient that is not simply governed by the ratio of the substrate covered by corrugation $\varphi$, which is taken to be the same for $r_{1}$ and $r_{1}^{\text {mod }}$ substrates. Instead, the edges of the corrugations influence the friction. This effect will be corroborated later in Sec. 4.2 for substrates with grooves.

In the end, we compare velocity profiles obtained for drops on corrugated substrates to the ones in Ref. [27] for droplets with contact angle $\theta_{\mathrm{E}} \approx 130^{\circ}$ pushed by a body force over a flat substrate. There, the velocity profiles are of the parabolic form along the whole distance $z$ to the substrate. In our case, we choose for comparison the data at solid-liquid interaction strength $\epsilon_{\mathrm{s}}=0.5 \epsilon$, as it provides the contact angle between $131.3^{\circ} \leqslant \theta \leqslant 134.0^{\circ}$ on the corrugated substrates $r_{1}$ and $r_{1}^{\bmod }$ (triangles in Fig. 4.7). The behavior that we observe, at least away from the substrate, is definitely well approximated by linear fits. Hence, we suggest that not only the contact angle determines the regime of the velocity profile $v_{x}(z)$ as proposed by Mognetti et al. [72], but the corrugation of the substrate also dictates the character of droplet's motion.

In this connection, it is interesting to notice that the friction at the surface is the dominant mechanism influencing the velocity of the droplet with the large contact angle (cf. Eq. 42 and discussion after it in [27]). Hence, the solid-liquid interface phenomena are 
crucial in understanding the difference in liquid motion at substrates of different topography. In the next section, we study the hydrodynamic properties of the solid-liquid interface as a function of the substrate corrugation and wetting parameters.

\subsection{Correlation between hydrodynamic properties of polymer liquid and surface topography}

Understanding the flow of liquids past solid surfaces has attracted abiding attention in micro- and nanofluidics. While on macroscopic scales, the non-slip or stick boundary, which assumes the velocity of the fluid at the surface coincides with that of the surface, is popular, this approximation may become invalid on smaller length scales pertinent to the operation of micro- and nanofluidic devices [100, 128, 129]. Controlling the friction at the interface between the solid substrate and the liquid is crucial in these applications, and different strategies have been pursued: Like in the case of wetting, one can control friction by (i) the direct, microscopic interactions between the solid and the liquid or (ii) the surface topography of the solid [110]. While the former is largely dictated by the chemistry of the solid and liquid, the latter alternative is expected to be a universal physical mechanism.

We consider a regularly corrugated surface topography as sketched in Fig. 4.3. A liquid on this structured substrate may either fill the cavities between corrugations (Wenzel state [118]) or build a straight liquid-vapor interface on the top of the corrugations (Cassie state [119] or fakir state), as discussed in Sec. 4.1.1. The macroscopic contact angle that a liquid drop makes with the solid substrate is dictated by the balance of surface tensions according to Young's equation [2]. The slippage of a liquid past a solid surface, in turn, is determined by a balance of viscous and frictional stresses at solidliquid boundary [103]. Modifying the topography of the surface, one simultaneously alters this balance and the surface tensions, therefore affecting both, slippage and wettability, respectively.

A dramatical decrease of slippage takes place for a liquid in Wenzel state with respect to the corresponding flat substrate [110-112]. The effective position of equivalent noslip wall was found between the top and bottom of the grooves [130]. In the case of the liquid in the Cassie (or fakir) state, in turn, the slip length increases and an enlargement by a factor of 2.5 in comparison to flat substrates has been observed [110]. Semi-analytical models, assuming a no-slip condition at the solid-liquid interface and infinite slip at liquid-vapor interface, have been developed to calculate an effective slip [102, 112].

Both, in the description of wetting and slippage, the phenomenological approaches exploited the scale separation between the geometry of the substrate (i.e., the shape 
of the corrugation) and the length scale that determines the surface tension or friction of the corresponding planar substrate. This assumption is justified for macroscopic corrugations but when the spatial scale of the corrugation decreases, geometry and interactions do no longer decouple; in this limit the surface roughness is an intrinsic property of the substrate. In this section, we use a particle-based simulations, which duly account for the discreteness of the fluid and its thermal fluctuations, to investigate the onset of deviations from the macroscopic phenomenological behavior.

Keeping the fraction $\varphi$ of the surface covered by grooves constant but varying their spatial dimensions, we investigate the influence of the roughness onto dynamical properties of the liquid in the Cassie and Wenzel states. Between these two states of the liquid, we only observe a gradual crossover but no phase transition [110]. We show that (i) the slippage at superhydrophobic substrates is significantly influenced by the additional friction at the edges of corrugations and (ii) that the hydrodynamic boundary position does not necessary coincide with a localization of the solid-liquid interface between the top and the bottom of the grooves.

There are two microscopic length scales of the polymer liquid to which the dimensions of the substrate topography can be compared: (i) the end-to-end distance $R_{\mathrm{ee}}=3.43 \sigma$ of the spatially extended chain molecules and (ii) the effective bead size $\sigma$. The former distance is related to the single-chain conformations in a cavity (cf. Fig. 4.8), while the latter sets the intrinsic scale of packing and layering effects in the fluid. For the short chain length considered in the present work, these two scales are not well separated.

Additionally, we compare the flat substrate composed of $X Y z$ unit cells and the flat substrate of $X Y Z$ unit cells used earlier in Chapter 3. During this comparison we refer to these substrates according to the notation of their unit cells. However, the terminus "flat substrate", used in this Chapter to compare flat and corrugated substrates, refers solely to $X Y z$ substrate, as the corrugations are constructed of the same building block - XYz unit cell.

The dimensions of the simulation box are $L_{x}=L_{y}=33 \sigma$, whereas $L_{z}$ is varied from $29.25 \sigma$ to $36.05 \sigma$ depending of the topography of the substrates and strength of solidliquid interaction $\epsilon_{\mathrm{s}}$. If not mentioned otherwise, $N_{0}=1920$ chains are confined in the simulation domain. The number of chains is varied in Sec.4.2.5, where we study flows at different pressures.

The results are summarized in the article submitted to Soft Matter.

\subsubsection{Transition from Cassie to Wenzel states}

To predict the stability region of the Wenzel state, we use a simple phenomenological continuum model [110], accounting for free energies of the interfaces presented in 


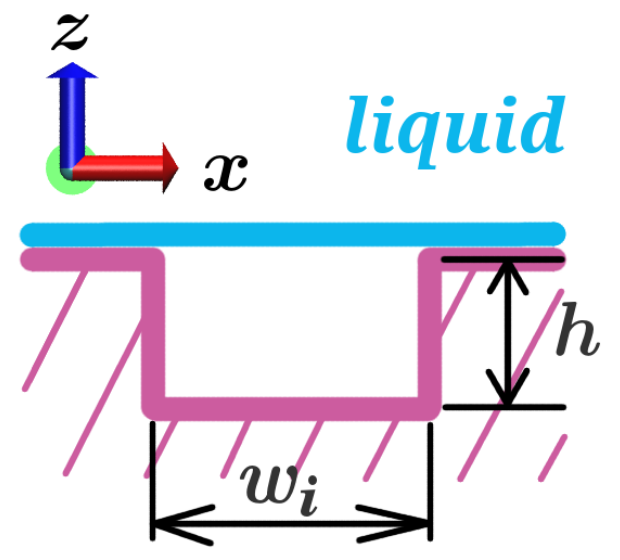

(a)

\section{liquid}

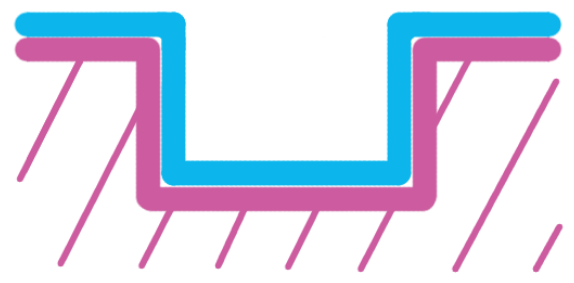

(b)

Figure 4.8: (a) Liquid in Cassie state does not fill the cavity, but creates straight liquidvapor interface of area $L_{y} w_{i}$. (b) Liquid in Wenzel state fills the cavity creating solid-liquid interfaces at the bottom and walls of the cavity.

Fig. 4.8. The liquid will fill the cavity if free energy of the liquid in the Wenzel state (Fig. 4.8b) is smaller than free energy of the liquid in the Cassie state (Fig. 4.8a):

$$
F_{\mathrm{W}}-F_{\mathrm{C}}<0
$$

From the geometries of interfaces the free energies can be written as

$$
\begin{gathered}
F_{\mathrm{C}}=\gamma L_{y} w_{i} \\
F_{\mathrm{W}}=\gamma_{\mathrm{SL}} L_{y} w_{i}+\gamma_{\mathrm{SL}} L_{y} 2 h=\gamma_{\mathrm{SL}} L_{y}\left(w_{i}+2 h\right),
\end{gathered}
$$

where $w_{i}$ is the width of the cavity and $\gamma_{\mathrm{SL}}$ is the solid-liquid surface tension that, in turn, is related to the contact angle of the liquid on a flat substrate $\theta_{\mathrm{E}}$ through Young's equation $\gamma_{\mathrm{SL}}=-\gamma \cos \theta_{\mathrm{E}}$. Here we recall that the density of the vapor is negligible. Therefore we assume a substrate-vapor surface tension of $\gamma_{\mathrm{SV}} \approx 0$. Then, the condition of filling the cavity by the liquid can be formulated in terms of the contact angle $\theta_{\mathrm{E}}$ as

$$
\theta_{\mathrm{E}}<\arccos \left(-\frac{1}{1+2 h / w_{i}}\right)
$$

Substituting $w_{i}$ with parameters of the substrates of our simulation model, we find that for a finely corrugated substrate the condition for filling the cavity corresponds to $\theta_{\mathrm{E}_{1}}<108^{\circ}$, and for a roughly corrugated one it holds $\theta_{\mathrm{E}_{2}}<124^{\circ}$. Comparing these phenomenological predictions with values of contact angles from Table. 4.1, we observe that the filling of cavities of finely and roughly corrugated substrates occurs at $\epsilon_{\mathrm{s}} \approx 0.6 \epsilon$ and $\epsilon_{\mathrm{s}} \approx 0.5 \epsilon$, respectively.

This phenomenological, macroscopic consideration predicts a first-order surface phase transition between the Cassie and the Wenzel state. In Fig. 4.9 we present the number density of liquid in a single cavity of the substrate for the two topographies. If 


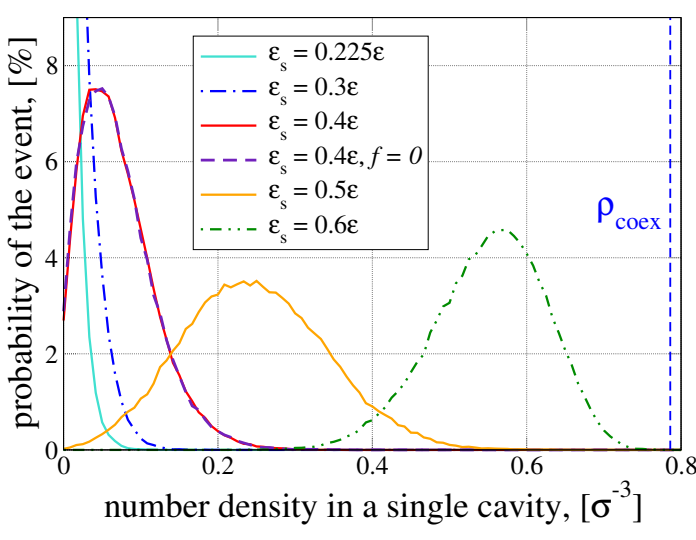

(a)

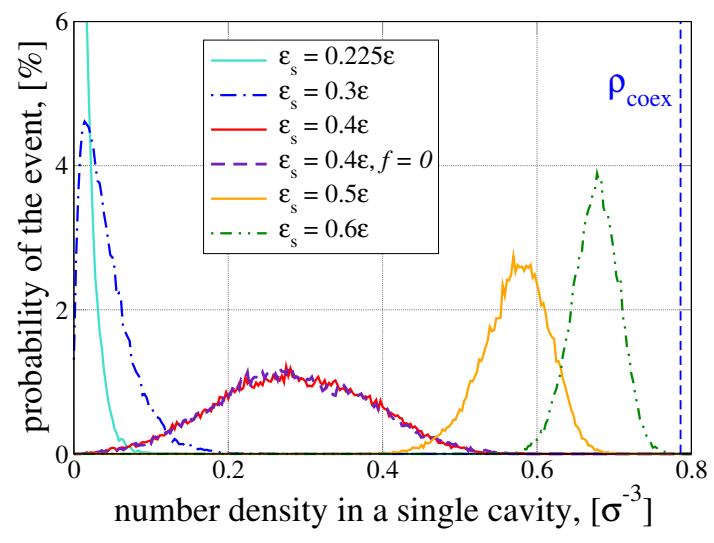

(b)

Figure 4.9: Histogram of a number density in a single cavity for finely (a) and roughly (b) corrugated substrates at varying strengths of solid-liquid interaction $\epsilon_{\mathrm{s}}$ with body force $0.001 \sigma / \tau^{2}$. Additionally plotted a histogram in the crossover regime at $\epsilon_{\mathrm{s}}=0.4 \epsilon$ without body force (dashed lines). The presence of a body force exerts only negligible influence on the density distribution. The coexistence density $\rho_{\text {coex }}$ is plotted by dashed vertical line.

there were a phase transition of first order between the Cassie and Wenzel states, one would expect a bimodal histogram, where the two peaks corresponded to empty cavities without liquid (Cassie state) and cavities completely filled by the liquid (Wenzel state), respectively. In marked contrast, however, we observe only a single peak in the density distribution. As we vary the solid-liquid interactions, $\epsilon_{\mathrm{s}}$, the average number density of liquid beads inside the cavity changes gradually. Around half-filling $\left(\rho_{\text {coex }} / 2 \approx 0.4 \sigma^{-3}\right)$ the distributions are very broad. The coexistence density of the liquid is indicated in the graph by the dashed vertical lines. The value inside the filled cavity is comparable but it remains smaller due to packing (layering) of the particle fluid inside the cavity. Snapshots reveal that the liquid-vapor interface strongly fluctuates in this crossover region and adopts states between straightly spanning the top of the grooves, as expected for the Cassie state, and touching the bottom of the groove, as being characteristic for the Wenzel state. In passing, we note that the application of the body force to the liquid does not change the behavior (cf. solid and dashed lines in Fig. 4.9 in presence and absence of a body force at $\epsilon_{\mathrm{s}}=0.4 \epsilon$, respectively).

There are two effects that contribute to the absence of a true surface phase transition in our simulations: (i) If the coupling between neighboring cavities were negligible because the cavities were widely separated, the number of particles in each groove would be an independent quantity. Then, since each groove would be a quasi-onedimensional system, there cannot be a true thermodynamic phase transition. Instead, we would expect to observe large but finite-sized domains of filled and unfilled portions along the groove. This rational is important for widely spaced and extremely long grooves, and this mechanism will eliminate all thermodynamic singularities at 
finite temperatures. While a systematic study of the filling/emptying behavior as a function of the spatial extension along the groove is beyond the scope of the present study, it is unlikely that this effect causes the absence of a sharp thermodynamic transition for the rather short grooves in our simulations because we do not observe multiple domains along a groove.

(ii) The absence of bimodal probability distributions in Fig. 4.9 rather indicates that even in the limit of infinitely long grooves, the transition between the Wenzel and the Cassie state is not of first-order but continuous. Thermal fluctuations give rise to fluctuations of the particle number in a cavity due to the finite compressibility of the liquid and, more importantly, due to fluctuations of local position of the liquid-vapor interface inside the groove. The latter are the analog of capillary waves in this confined geometry. For the geometry used in our simulation study, the height of the grooves is on the same order of magnitude as the intrinsic width of the liquid-vapor interface, and already small excursions of the interface position result in a significant change of the filling fraction. Even if $h / \sigma$ is not very small but the grooves are widely separated to be considered independent, we anticipate large fluctuations because the macroscopic consideration, Eq. (4.13), asserts that the Wenzel and Cassie state have equal free energies for $\theta_{\mathrm{E}} \rightarrow 90^{\circ}$. In this case, the liquid-vapor interface can move homogeneously like a rigid plane up and down in the groove. In fact, these simple macroscopic considerations are in agreement with a non-mean-field analysis [131, 132].

If the liquid is confined into a channel, the pressure might deviate from the vapor pressure, at which the liquid and vapor of vanishingly small density coexist. The associated effects will be discussed in the following section.

\subsubsection{Normal pressure in the bulk phase}

We study a polymer liquid that is confined between two apposing substrates. A flat unstructured substrate, a finely corrugated substrate with roughness $r_{1}$ and a substrate with a rough corrugation, $r_{2}$, are considered. All of them are composed of $X Y z$ unit cells, introduced in Sec. 2.1. Additionally, we vary the strength of solid-liquid interaction, $\epsilon_{\mathrm{s}}$. To adjust the normal pressure to its coexistence value, $p_{\text {coex }} \approx 0$, we change the distance $L_{z}$ between the two apposing substrates and calculate the corresponding pressure inside the liquid composed of $N_{0}=1920$ chains. The resulting width, $L_{z}^{\text {coex }}$, is therefore a function of solid-liquid interaction strength $\epsilon_{\mathrm{s}}$, the corrugation $r_{i}$, and the number of chains $N_{0}$, i.e., $L_{z}^{\text {coex }}=L_{z}^{\text {coex }}\left(\epsilon_{\mathrm{s}}, r_{i}, N_{0}\right)$.

We calculate the normal pressure tensor component $p_{\mathrm{n}}[42,43]$ dividing the systems into slabs perpendicular to the direction of the substrates and using the approach by Irving and Kirkwood [44], as discussed in Sec. 3.1.1. The pressure is divided into a configurational term, which is proportional to the number density $\rho(k)$ in a slab $k$, and 


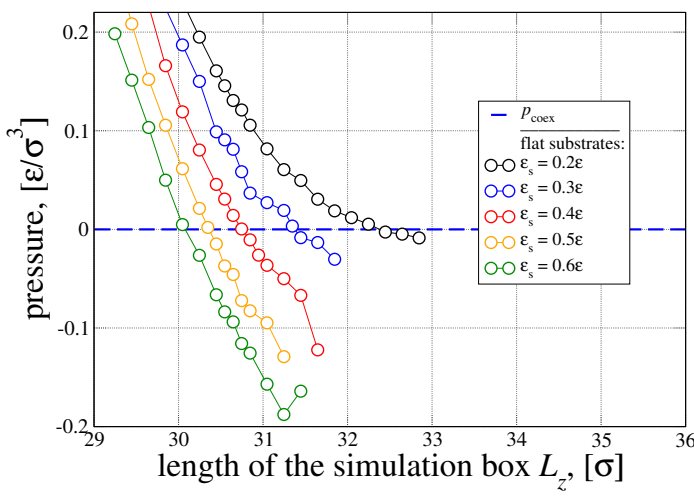

(a)

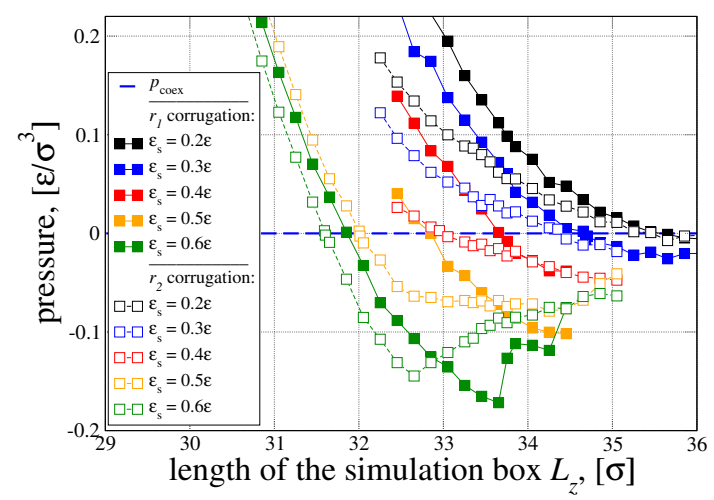

(b)

Figure 4.10: Pressure of the polymer liquid at the center of the film. Panel (a) shows the results for a flat surface, while panel (b) presents the results for the two corrugated substrates. Filled symbols represent the corrugation $r_{1}$ and empty symbols correspond to $r_{2}$. Dashed horizontal lines indicate the coexistence density and pressure, respectively.

a virial term:

$$
p_{\mathrm{n}}(k)=k_{\mathrm{B}} T\langle\rho(k)\rangle+\frac{1}{V_{\mathrm{sl}}}\left\langle\sum_{i<j}^{(k)} \frac{z_{i j}^{2}}{r_{i j}}\left|\mathbf{F}_{i j}\right| \cdot \cos \left(\widehat{\mathbf{F}_{i j}, \mathbf{r}_{i j}}\right)\right\rangle,
$$

where $V_{\mathrm{sl}}$ denotes the volume of the slab, $x_{i j}, y_{i j}, z_{i j}$ are the distances between interacting particles $i$ and $j$ in $x, y, z$ directions, respectively, and $r_{i j}^{2}=x_{i j}^{2}+y_{i j}^{2}+z_{i j}^{2}$. The angle between the force $\mathbf{F}_{i j}$ and the distance vector $\mathbf{r}_{i j}$ is given by expression $\widehat{\mathbf{F}_{i j}, \mathbf{r}_{i j}}$, and its cosine can take the values \pm 1 . Angular brackets $\langle\cdots\rangle$ denote averages in the canonical ensemble. The sum $\sum_{i<j}^{(k)}$ runs over particles $i$ and $j$ if a portion of line connecting them is located inside the slab $k$. The interactions between the solid substrate and the liquid particles also contribute to the virial.Since the solid-liquid interaction is of finite range, however, they do not contribute to the pressure in the center of the confined film. Mechanical stability asserts that the pressure normal to the surfaces does not depend on position and thus equals the pressure at the center of the film.

The dependence of the normal pressure on the distance, $L_{z}$, between the walls is presented in Fig. 4.10. At small $L_{z}$, the density and pressure at the center of the film monotonously decrease with increasing $L_{z}$. At constant number of particles, we observe that the higher the attractive strength of solid-liquid interaction, $\epsilon_{\mathrm{s}}$, is the smaller is $L_{z}^{\text {coex }}$, because of the excess of the compressible liquid at the attractive substrate. $L_{z}$ is defined as the maximal distance between the corrugated substrates, i.e., measured from the bottoms of the corrugation. $L_{z}^{\text {coex }}$ is larger for a finely corrugated substrate with roughness $r_{1}$ than for the coarse grooves (filled vs. open symbols in Fig. 4.10), because the polymers explore the wider grooves better, and thereby they increase the liquid excess at the substrate and reduce the pressure at the center.

At large wall separation, $L_{z}>32.5 \sigma$, we find deviations from the expected monotonous 


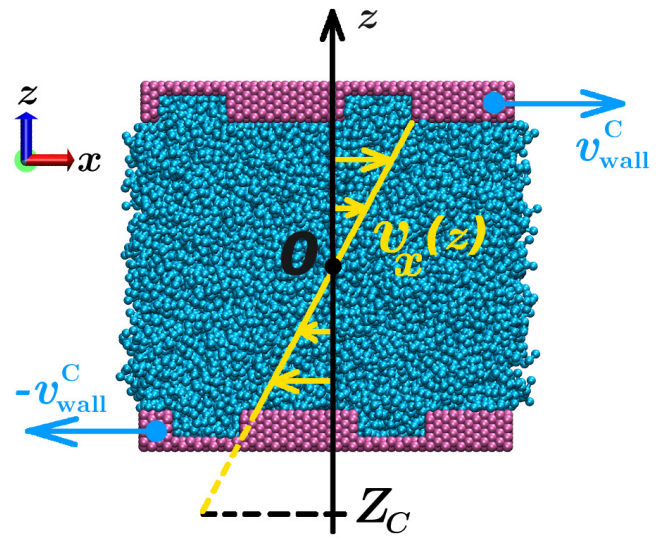

(a)

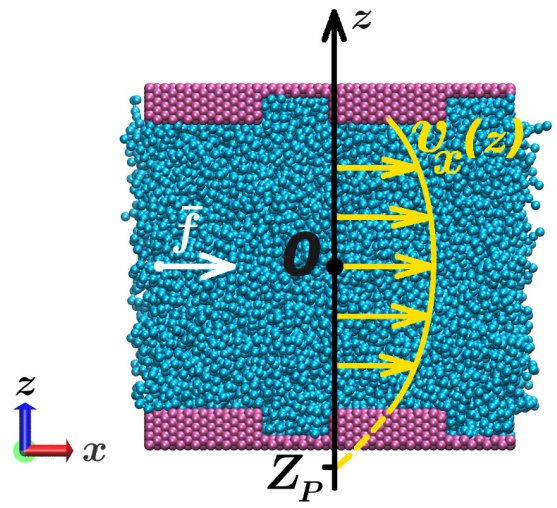

(b)

Figure 4.11: (a) Couette flow created in the system with roughly corrugated substrates. Two walls are moved into opposite directions with absolute velocities $v_{\text {wall }}^{C}$. The origin of the $z$-axis is taken in the middle of the channel (black). The linear velocity profile is sketched by the arrows of different size. The interpolated profile reaches the velocity of the wall at the coordinate $Z_{C}$. (b) The flow created by application of a body force $\vec{f}$ (white) onto the particles of the liquid. The parabolic velocity profile (arrows) is interpolated to the velocity of the wall $\left(v_{\text {wall }}^{\mathrm{P}}=0 \sigma / \tau\right)$ and reaches it at $Z_{\mathrm{P}}$.

dependence on $L_{z}$ due to the formation of vapor bubbles under tensile stress, $p<$ 0 . These cavitation events nucleate more readily at strongly attractive surfaces $\epsilon_{\mathrm{s}} \geqslant$ $0.5 \epsilon$ and for corrugated substrates. In those cases, the simulations did not necessarily achieve equilibrium.

\subsubsection{Comparison of hydrodynamic boundary positions}

Here we study the flow of a polymer liquid past corrugated substrates. Therefore we expect a finite slip length, $\delta$, and the effective location, $z_{\mathrm{h}}$, at which the hydrodynamic boundary conditions is to be applied, is not obvious. Since Navier's boundary condition features two independent parameters, as was shown in Sec. 3.3, we use two flow set-ups: Couette flow, generated by moving the surfaces, and Poiseuille-like flow, generated by applying an external body force on the liquid. The linear and parabolic velocity profiles expected from the macroscopic continuum model for Couette and Poiseuille flow, respectively, are illustrated in Fig. 4.11.

The set up of the Couette flow is the same, as previously in Sec. 3.3. We move the two apposing substrates into opposite directions with a small absolute velocity $v_{\text {wall }}^{\mathrm{C}}=$ $0.075 \sigma / \tau$ (Couette flow) as shown in Fig. 4.11a. The average shear rate is defined as a ratio of the velocity of the wall $v_{\text {wall }}^{\mathrm{C}}$ to the width of the channel $L_{z}$ and, in our simulations, it is of the order of $\dot{\gamma} \sim 5 \cdot 10^{-3} \tau^{-1}$.

To generate Poiseuille-like flow, we impose body forces $f$ of strength $0.001 \sigma / \tau^{2}, 0.003 \sigma / \tau^{2}$, 


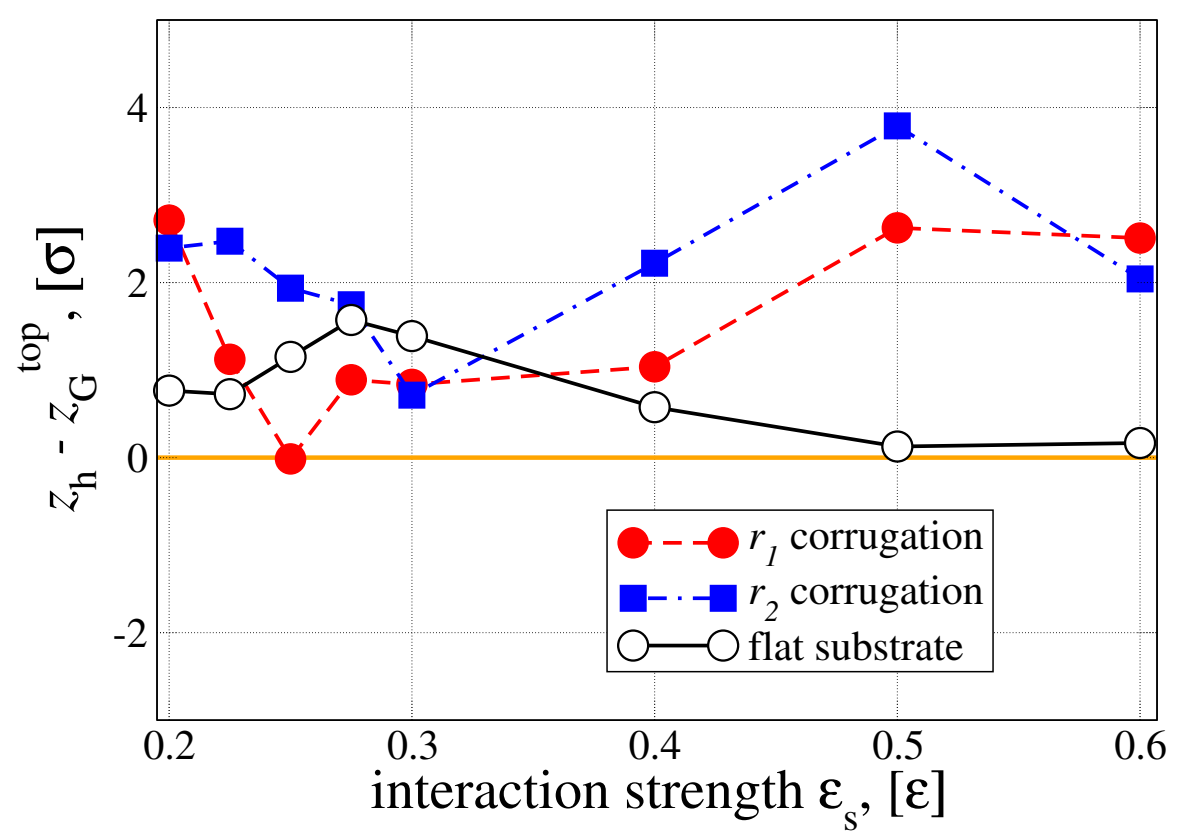

Figure 4.12: Distance of the position, $z_{\mathrm{h}}$, at which Navier's boundary condition 3.36 is to be applied, to the innermost substrate atom $z_{\mathrm{G}}^{\text {top }}$ for channels with fine and rough corrugations (lines with filled circles and squares, respectively) and for channels with flat walls (line with open circles). Positive values, $z_{\mathrm{h}}-z_{\mathrm{G}}^{\text {top }}>0$, indicate that the effective boundary position is closer to the center of the channel than any particle of the wall.

$0.005 \sigma / \tau^{2}$ and $0.008 \sigma / \tau^{2}$. An external force injects energy into the system, which is removed by the thermostat, keeping the temperature constant. In order to preserve a linear response of the liquid, only the smallest force was applied at small values of $\epsilon_{\mathrm{s}}$, whereas for $\epsilon_{\mathrm{s}} \geqslant 0.4 \epsilon$ all four forces were used.

Using the simulation data obtained from Couette and Poiseuille-like flows at $p_{\text {coex }} \approx 0$, we can independently determine the slip length, $\delta$, and the position of the hydrodynamic boundary, $z_{\mathrm{h}}$. In Fig. 4.12 we present the distance between the boundary position $z_{\mathrm{h}}$ and the position, $z_{\mathrm{G}}^{\text {top }}$, of the innermost substrate segment, which is located on the tops of ridges between the grooves. In case of a flat substrate (black line with open circles), the position $z_{\mathrm{G}}^{\text {top }}$ refers to the innermost atomic layer of the substrate. It is interesting to notice, that even in the case of flat walls and small $\epsilon_{\mathrm{s}}$, the hydrodynamic boundary is found somewhat inside the liquid, but not directly at the solid-liquid interface. For most parameters at structured substrates, the hydrodynamic boundary is located $2-4$ segment diameters inside the liquid above the top of the ridges between the grooves. This observation for our molecular fluid even holds in the Wenzel state, where the liquid enters the grooves.

The position of an effective no-slip plane, where the extrapolation of the hydrodynamic velocity field, vanishes $-Z_{C}$ for Couette flow and $Z_{P}$ for Poiseuille flow - will be located at an intermediate coordinate between the peaks and valleys of the substrate topogra- 


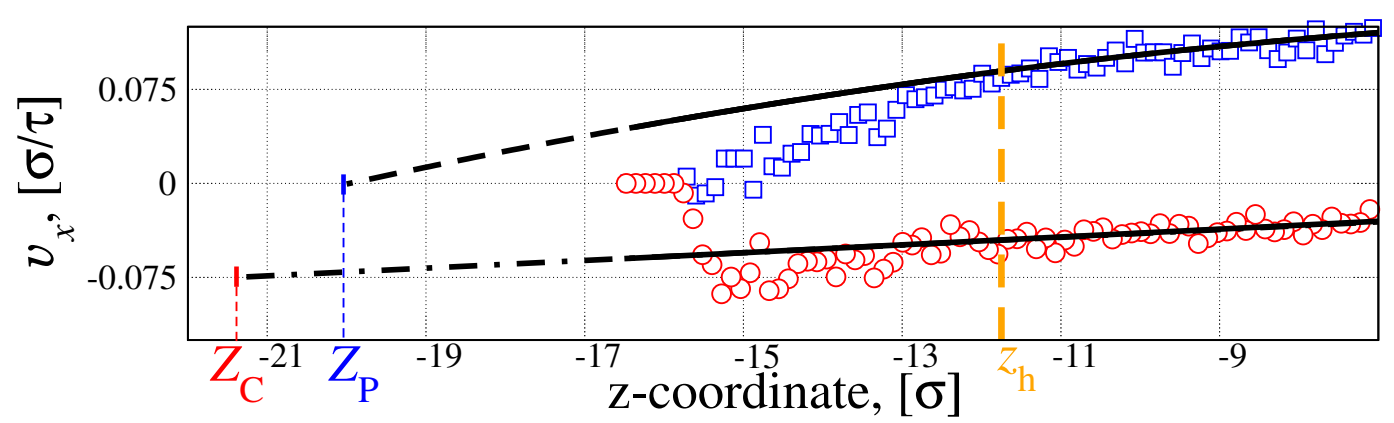

Figure 4.13: Velocity profiles of Couette flow (circles) and Poiseuille-like flow with body force $f=0.005 \sigma / \tau^{2}$ (squares) at $\epsilon_{\mathrm{s}}=0.4 \epsilon$ and rough corrugation $r_{2}$ are shown. The case corresponds to the crossover regime, when liquid enters the cavity. Smooth thick solid lines are the fits to the velocity profiles, measured away from the substrate. The positions $Z_{\mathrm{C}}$ and $Z_{\mathrm{P}}$, where extrapolated macroscopic profiles reach the velocity of the wall, $v_{\text {wall }}^{\mathrm{C}}=-0.075 \sigma / \tau$ and $v_{x}^{\mathrm{P}}\left(-Z_{\mathrm{P}}\right)=0$ for Couette and Poiseuille-like flows, respectively, are indicated. These no-slip positions depend on the type of the flow and therefore cannot be used as a boundary condition.

phy in the Wenzel state if the slip length, $\delta$, is larger than $2-4$ segment diameters. This result is compatible with lattice Boltzmann simulations of a continuum fluid past statistically rough surfaces [130]. We emphasize, however, that the no-slip position depends on the flow and, hence, cannot be used to parameterize a boundary condition, as shown in Fig. 4.13. Moreover, upon reaching the limit $\theta_{\mathrm{E}} \rightarrow 90^{\circ}$ the slippage can be less than 2 segment diameters (cf. section 4.2.4), and the corresponded no-slip position shifts into the liquid.

In Fig. 4.14 we present the mass-weighted, two-dimensional velocity profiles for corrugated substrates at $\epsilon_{\mathrm{s}}=0.4 \epsilon$. Additionally, we demonstrate the velocity profiles $v_{x}$ (squares) and their macroscopic fits (smooth solid lines) measured away from the substrate to neglect distortion by the friction at the boundary. The position of the hydrodynamic boundary condition is also indicated in the graph (horizontal dashed line). In both cases, it is above the corrugation. Qualitatively, $z_{\mathrm{h}}$ describes the crossover between two behaviors: For larger distances from the substrate, the velocity is strictly parallel to the $x$-axis as predicted by the Navier-Stokes equation for Poiseuille and Couette flow past a macroscopic substrate, while closer to the substrate, the velocity field acquires a spatially periodic, perpendicular z-component. In agreement with detailed hydrodynamic calculations of flow past a corrugated substrate, the amplitude of the perpendicular velocity component will decay faster away from the substrate if the lateral period is smaller [102]. Additionally, we note that the deviations of the velocity profiles from the macroscopic fits begin at the vicinity of hydrodynamic boundary position.

Finally, we mention that, at $\epsilon_{\mathrm{s}}=0.4 \epsilon$ (crossover regime, when the liquid enters the cavity but not yet in the macroscopic Wenzel state), the average velocity of the liquid in 


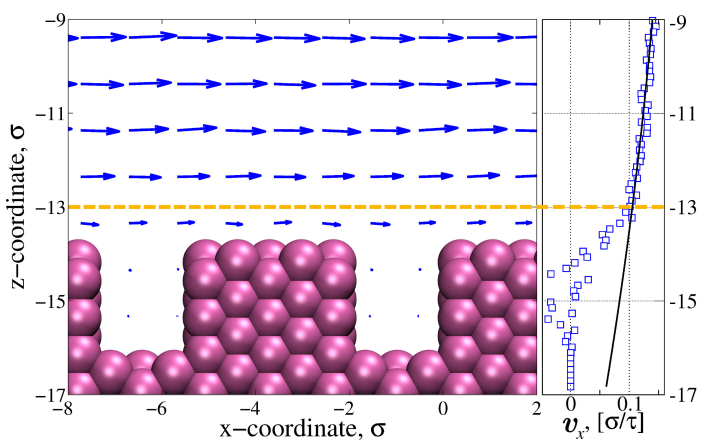

(a)

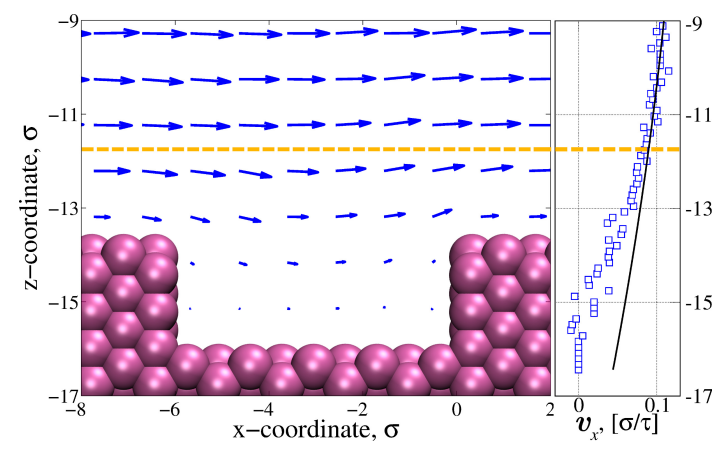

(b)

Figure 4.14: Mass flux, $\rho \vec{v}$ (arrows), in the $x z$ plane at $\epsilon_{\mathrm{s}}=0.4 \epsilon$ and $w_{1}=2.06 \sigma$ in panel (a) and at $\epsilon_{\mathrm{s}}=0.4 \epsilon$ and $w_{2}=6.18 \sigma$ in panel (b) under body force $f=0.005 \sigma / \tau^{2}$ (Poiseuillelike flows). Both cases, (a) and (b), correspond to the crossover regime, when liquid enters the cavity. In the accompanying plots velocity profiles (squares) and the extrapolated hydrodynamic profile (thick solid line) are shown.Smooth solid lines are the fits to the velocity profiles, measured away from the substrate. The position of the hydrodynamic boundary, $z_{\mathrm{h}}$, is indicated by horizontal dashed lines.

a cavity of the finely corrugated substrate nearly vanishes, whereas in case of a rough corrugation the liquid actually flows in the cavity. This effect may influence the local shear stress and the effective boundary properties.

\subsubsection{Comparison of slip length and surface friction}

In this section we apply the same procedure to compare the slip lengths and friction coefficients of flat and corrugated substrates, as was used in Sec. 3.3. The second parameter of the hydrodynamic boundary condition is the slip length, $\delta$, or, equivalently, the friction coefficient $\lambda=\eta / \delta$. Employing the dependence of the slip length on solidliquid interaction strength $\delta \sim 1 / \epsilon_{\mathrm{s}}^{2}$ [105], we firstly compare the $X Y z$ flat substrate used in this Chapter with $X Y Z$ substrate, used in Chapter 3. The comparison is made at $p_{\text {coex }} \approx 0$ in the bulk of the liquid.

Since the orientation of the unit cell of the flat crystalline substrates differs, we observe that the slip length for $X Y z$ substrate is smaller than for the previously used $X y Z$ substrate (circles vs. squares in Fig. 4.15, respectively). Such an orientation dependence of slippage has also been observed in other studies [133]. The reason is that for the $X Y Z$ substrate the atoms are packed more tightly in one atomic layer, than in the case of $X Y Z$ substrate. Indeed, the lengths of the lattice vectors $\vec{y}$ differ by a factor of $\sqrt{3}$. Hence, the $X y Z$ substrate is more uniform (more "flat") than the $X Y Z$ one.

To compare the hydrodynamic boundary conditions of flat and structured substrates composed of the same unit cells of $X Y z$ type, we aim to adjust the pressure to its coexistence value, $p_{\text {coex }} \approx 0$, such that the liquid-vapor interface in the Cassie state hor- 


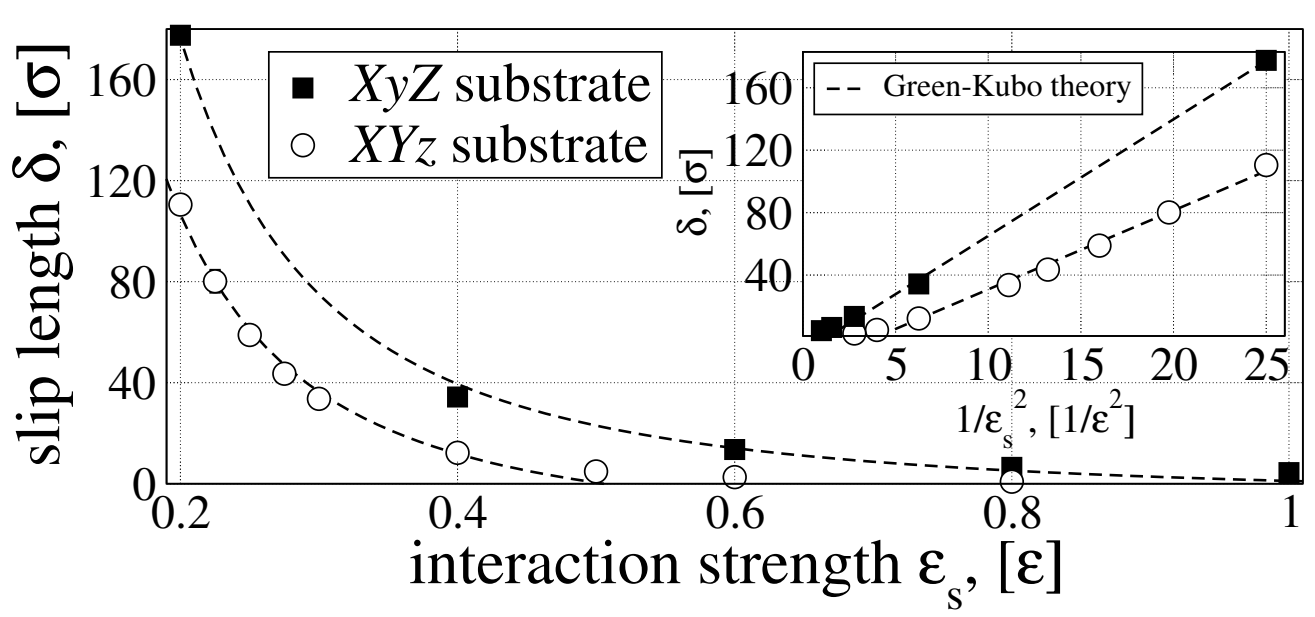

Figure 4.15: The slip length $\delta$ for a liquid confined between flat substrates of $X Y Z$ (squares) and $X Y Z$ (circles) types. The dashed lines are the analytical predictions of Barrat and Bocquet [105] based on Green-Kubo relation for the friction coefficient.

izontally spans the grooves. Then, one expects that the friction in the Cassie state is reduced, $\lambda=\varphi \lambda_{\text {flat }}$, compared to its value on a flat substrate, where $\varphi$ is the fraction of the substrate area in contact with the liquid [110].

In the Wenzel state, the macroscopic expectation is that the friction, like the surface free energy, is proportional to the microscopic contact area, $r$, between liquid and substrate. Thus a finely corrugated substrate, $r_{1}=1.865$ is expected to give rise to more friction (with respect to the projected substrate area) than a coarsely corrugated one, $r_{2}=1.289$.

Fig. 4.16a presents the slip length, $\delta$, as a function of the solid-liquid attraction. The qualitative behavior is similar for flat and corrugated substrates. For strong attraction, the slip lengths is microscopic but upon approaching $\epsilon_{\mathrm{s}} \rightarrow 0$ (purely repulsive substrate), the contact angle approaches $180^{\circ}$ (drying transition) and a lubricating thin vapor layer intervenes between the substrate and liquid giving rise to large slippage.

When plotting $\lambda \sim 1 / \delta v s . \epsilon_{\mathrm{s}}^{2}$, one expects to find that the simulation data are compatible with two linear relations with different slopes. One linear relation corresponds to the Wenzel state for large $\epsilon_{\mathrm{s}}$, and another linear relation with a larger slope characterizes the Cassie regime, where the substrate has less contact with the liquid. In our simulation, however, the crossover gradually occurs in the wide range of $\epsilon_{\mathrm{s}} / \epsilon \in(0.275 ; 0.6)$ or $\epsilon_{\mathrm{s}} / \epsilon \in(0.25 ; 0.5)$ for the finely and coarsely corrugated substrate. Only at $\epsilon_{\mathrm{s}}=0.6 \epsilon$ or $\epsilon_{\mathrm{s}} \geqslant 0.5 \epsilon$, respectively, we observe the system consistently in the Wenzel state. Therefore, we cannot identify the linear behavior that marks the Wenzel state.

In the crossover region, the friction coefficients of both substrates do not significantly differ as shown in Fig. 4.16b. According to macroscopic considerations, we expected the finely corrugated substrate to generate more friction because of the larger contact area with the fluid. In our simulations, however, the liquid is rather trapped inside the 


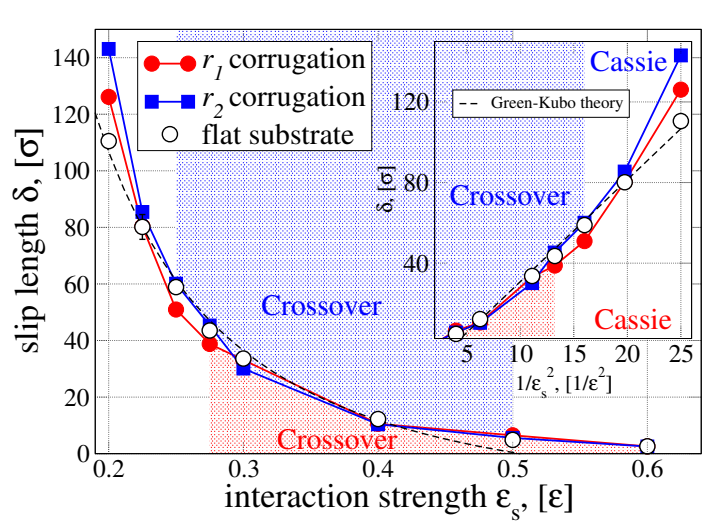

(a)

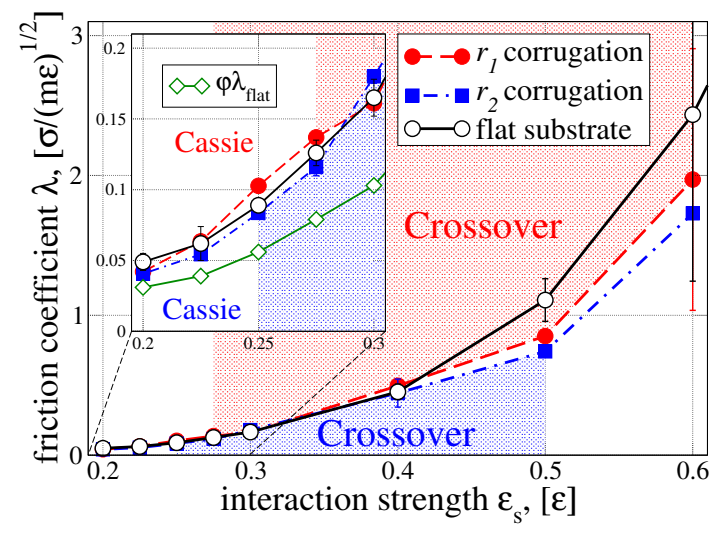

(b)

Figure 4.16: (a) The slip length $\delta$ for a liquid at flat (open circles) and patterned substrates of $r_{1}$ and $r_{2}$ corrugations (filled circles and squares, correspondingly) at coexistence pressure. The inset shows the slip length $\delta$ as a function of inverse squared interaction strength $\epsilon_{\mathrm{s}}$. (b) Friction coefficient $\lambda$ for different topographies of the substrate. The line with diamonds in the inset corresponds to the friction at the flat substrate multiplied by the area fraction $\varphi=$ 0.625 covered by grooves. It is smaller than measured friction coefficients for corrugated substrates due to the friction at the edges of the grooves. The crossover regions, separating Cassie and Wenzel states, are shown by shaded areas for the different corrugations.

finely corrugated grooves, and the friction is not predominately generated at the solidliquid interface but by viscous dissipation due to the velocity gradient at the interface between the trapped liquid inside of the groove and the flowing liquid at the center.

In the Cassie state, the friction of the corrugated substrate is comparable to that of the flat substrate. The macroscopic prediction that the friction is reduced by a factor of $\varphi=0.625$ is not observed in our model. Moreover, the friction on the finely corrugated substrate is slightly higher than on the coarsely corrugated one. This effect can be partially rationalized by the effect of edges.

In Fig. 4.17 we present two-dimensional density plots in the vicinity of the corrugated substrate. We observe that density oscillations, which indicate layering effects of the particle fluid, emerge from the edges of the grooves and propagate a few particle diameters, $\sigma$, into the channel. We also note that even for the rather small body force, $f=0.005 \sigma / \tau^{2}$, that drives the fluid through the channel, the flow breaks the left-right symmetry, and the packing effects at the left and right edges of a groove differ slightly. The edge facing the flow (on the right side of the cavity) exhibits stronger layering effects than the edge on the left side.

To a first approximation, we try to quantify these deviations from the macroscopic prediction due to localized perturbations of the flow by augmenting the Cassie model of friction by a term that accounts for additional friction at the edges of the grooves.

$$
\lambda=\varphi \lambda_{\text {flat }}+K\left(\epsilon_{\mathrm{s}}, d, w\right) \Sigma_{\text {edge }}
$$




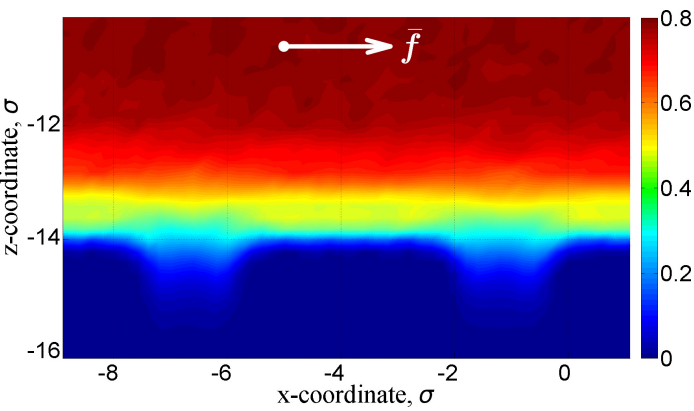

(a)

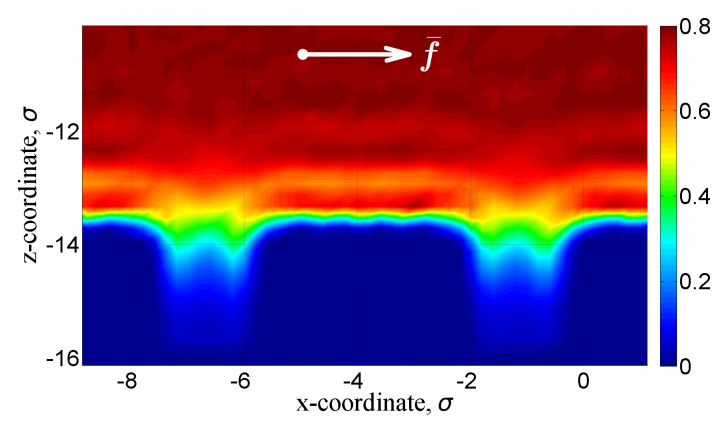

(c)

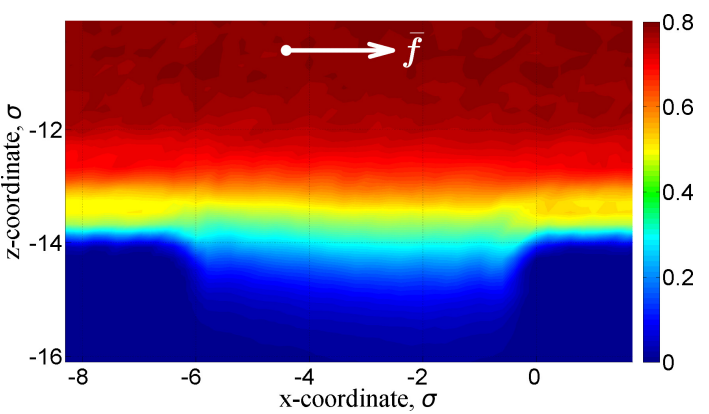

(b)

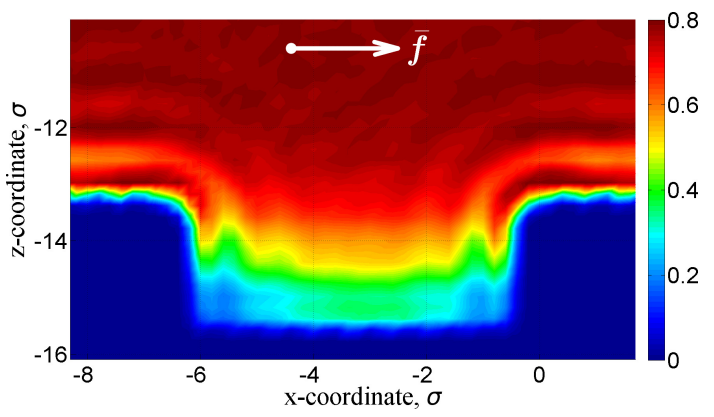

(d)

Figure 4.17: Two-dimensional map of the number density of the particle liquid in the vicinity of the finely (left column) and coarsely (right column) corrugated substrate for $\epsilon_{\mathrm{s}}=0.3 \epsilon$ (top row) and $\epsilon_{\mathrm{s}}=0.4 \epsilon$ (bottom row). A body force $f=0.005 \sigma / \tau^{2}$ generates a Poiseuillelike flow whose direction is indicated by the arrow.

where $\Sigma_{\text {edge }}=\frac{2}{d+w}$ is the line density of edges and $K\left(\epsilon_{\mathrm{s}}, d, w\right)$ quantifies the additional friction coefficient per edge length. $K$ slightly depends on the geometry but in the limit of isolated edges, $d, w \rightarrow \infty$, we expect $K$ to adopt a finite value. The line density of edges is three-fold higher for the finely corrugated substrate than for the coarsely corrugated one. From the data of Fig. $4.16 \mathrm{~b}$ we extract the effective edge friction $K$. The values are in the range of $0.03 \leqslant K \leqslant 0.16$ for the finely corrugated substrate and somewhat larger, $0.09 \leqslant K \leqslant 0.22$, for the wider grooves. The order of magnitude of the edge friction, $K \sim 2.5 \lambda_{\text {flat }} \sigma$, is plausible, i.e., each edge generates an additional friction that corresponds to roughly two additional rows of the substrate. The increase of $K$ with increasing the distance between the edges is in accord with Fig. 4.17, where we observe that liquid layering effects are more pronounced for wider grooves than for more narrow ones.

\subsubsection{Pressure-driven flow in patterned channels}

For an incompressible liquid confined into a channel with flat boundaries, the flow that is generated by a body force and pressure-driven Poiseuille flow are equivalent. For corrugated substrates, however, both methods of generating flow are no longer 


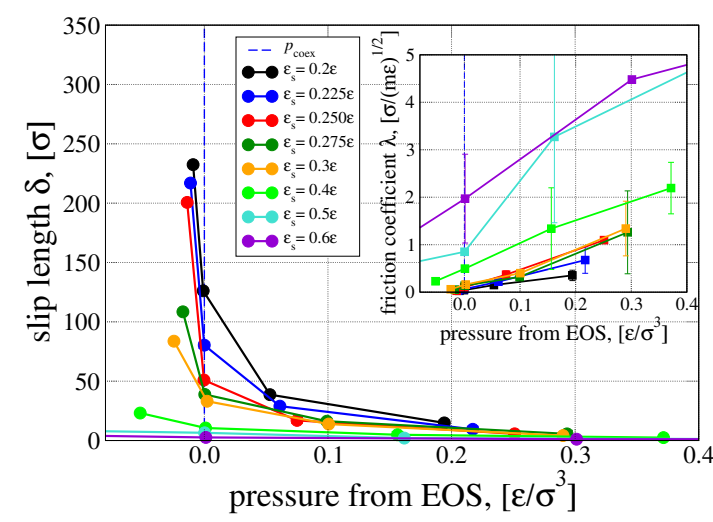

(a)

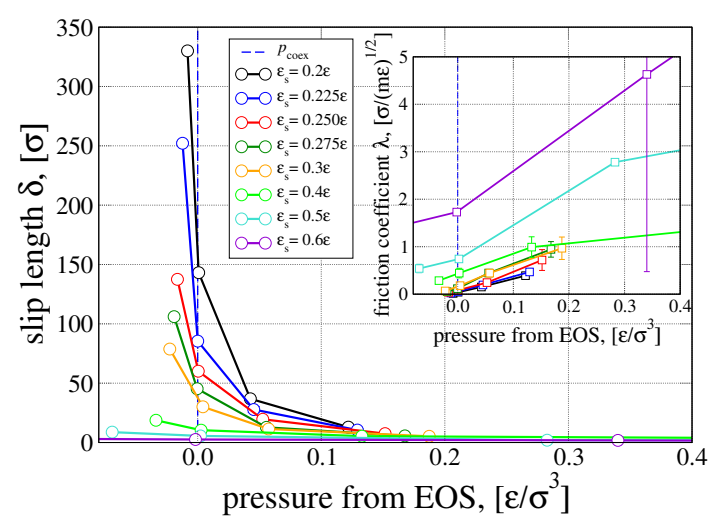

(b)

Figure 4.18: Slip length, $\delta$, as a function of the pressure measured from EOS of the channel confined by finely (a) or coarsely (b) corrugated substrates, respectively. The insets present the friction coefficient, $\lambda$, and the vertical line marks $p_{\text {coex }}$. The widths of the channels $L_{z}$ at fixed $\epsilon_{\mathrm{S}}$ and corrugation $r_{i}$ correspond to the widths $L_{z}^{\text {coex }}\left(\epsilon_{\mathrm{S}}, r_{i}, N_{0}\right)$, calculated in Sec. 4.2.2.

equivalent: If we use a body force to set the fluid in motion, the system will remain translationally invariant along the flow direction, and the pressure at the center of the channel will be independent from the position along the channel. If we generate the flow by a pressure gradient, instead, the pressure will decrease along the channel. Even if the liquid were incompressible and hence its shear viscosity would not depend on pressure, we expect that the parameters of the hydrodynamic boundary condition of a corrugated substrate - slip length, $\delta$, and position, $z_{\mathrm{h}}$ - will depend on the pressure and therefore will vary along the channel. The ability of the fluid to explore the cavities of the substrate makes the total system effectively compressible. Upstream, where the pressure is high, the fluid is more likely to enter the cavities of the substrate (Wenzel state), and the friction will be high. Farther downstream, in turn, the pressure is low and the liquid is more likely to adopt the Cassie state, which results in a lower friction. At negative pressures, $p<p_{\text {coex }}$, one might expect either again an increase of friction because the liquid-vapor interface in the Cassie state is curved (bubble mattress) [134] or a decrease of friction because the vapor layer at the solid is formed and acts as a perfect lubricant $[110,135]$.

In order to study how the slip length depends on pressure, we generate Poiseuillelike flow via a body force and vary the number of polymers inside the slit-pore. We measure the density at the center of the channel and use the bulk equation of state (EOS, cf. Appendix A) to determine the pressure.This pressure corresponds to the normal pressure inside the channel. The simulation results in Fig. 4.18 demonstrate that the slip length sensitively depends on pressure.

If the pressure is high, the slip length, $\delta$, is microscopically small and the friction coeffi- 


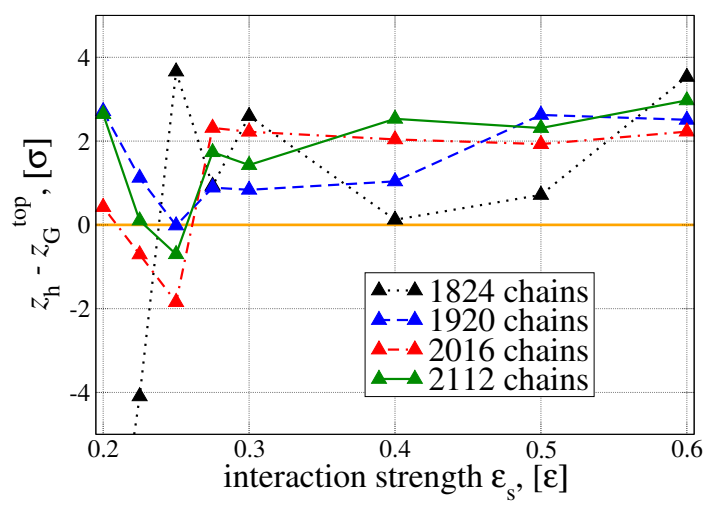

(a)

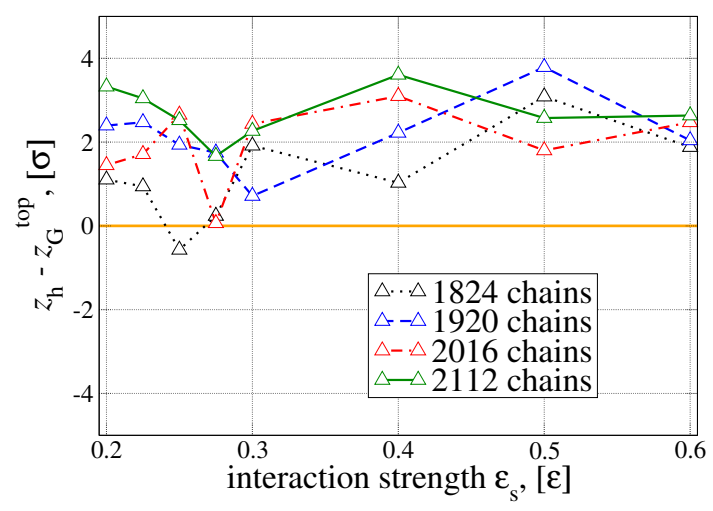

(b)

Figure 4.19: Distance of the position, $z_{h}$, at which Navier's boundary condition 3.36 is to be applied, to the innermost substrate atom $z_{\mathrm{G}}^{\text {top }}$ for channels with fine (a) and rough (b) corrugations, respectively. Positive values, $z_{\mathrm{h}}-z_{\mathrm{G}}^{\text {top }}>0$, indicate that the effective boundary position is closer to the center of the channel than any particle of the wall. Different line types correspond to different number of polymers in a channel at fixed $L_{z}=L_{z}^{\text {coex }}\left(\epsilon_{\mathrm{s}}, r_{i}, N_{0}\right)$, giving rise to different pressures.

cient $\lambda$ is very high. In this case we observe a parabolic profile with an effective no-slip boundary condition. Upon reducing the pressure, the slip length increases. When approaching the coexistence pressure, the slip length increases for weakly attractive substrates because the grooves are gradually emptied. At $p_{\text {coex }}$ the liquid adopts the Cassie state. For stronger attractions, $\epsilon_{\mathrm{s}}$ the liquid remains in the Wenzel state even when we approach $p_{\text {coex }}$. The continuous transition between Cassie and Wenzel states at $p_{\text {coex }}$ as a function of $\epsilon_{\mathrm{s}}$ has been studied in Sec. 4.2.1. For $p<p_{\text {coex }}$ very large slip lengths can be achieved because a thin vapor layer is formed at the substrate that acts as lubricant. In this case, we observe plug flow because the slip lengths exceeds our system size, $L_{z}$ by far, and the friction is very small. Such a lubrication layer forms more readily for small $\epsilon_{\mathrm{s}}$.

The dependence of the hydrodynamic boundary position, $z_{\mathrm{h}}$, on pressure is shown in Fig. 4.19. As in the case of flows at coexistence pressure, discussed in Sec. 4.2.3, for most parameters the hydrodynamic boundary is found inside the liquid above the top of the ridges between the grooves. The significant change of hydrodynamic position, $z_{\mathrm{h}}$ at low pressures and at finely corrugated substrate, which goes along with a large statistical uncertainty, may be explained by the formation of a lubricant layer of vapor at the solid boundary and the concomitant, large increase of slippage (plug flow).

Thus slippage and friction greatly depend on the pressure inside the channel, and the pressure dependence may significantly affect the flow profile across the channel and the pressure drop along the channel. Using Navier's hydrodynamic boundary condition, we compute how the flow rate, $Q$, depends on the slip length, $\delta$, and half of the 
effective channel width, $z_{\mathrm{h}}$

$$
Q=2 \int_{0}^{L_{y}} d y \int_{0}^{z_{\mathrm{h}}} v_{x}^{\mathrm{P}}(z) d z=-\frac{2 L_{y} z_{\mathrm{h}}^{3}}{3 \eta} \frac{\mathrm{d} p}{\mathrm{~d} x}\left[3 \frac{\delta(p)}{z_{\mathrm{h}}}+1\right]
$$

where $\frac{d p}{d z}$ is the local drop of pressure along the channel. Mass conservation requires that $Q$ be constant along the channel and the pressure profile along the channel is dictated by

$$
\frac{\mathrm{d} p}{\mathrm{~d} x} \sim-\frac{1}{3 \frac{\delta(p(x))}{z_{\mathrm{h}}}+1}
$$

If the slip length is constant along the channel, the pressure decreases linearly with the coordinate, $x$, along the channel. For the corrugated substrate, however, $\delta$ increases with $p$ and, consequentially, the pressure decreases rapidly upstream and more slowly downstream. In the case of negative pressures at the end of the channel (suction) and small $\epsilon_{\mathrm{s}}$, we expect plug flow with vanishingly small pressure drop.

\subsection{Discussion}

In this chapter we studied droplets on symmetrically structured substrates and flows of a polymer liquid in channels with patterned walls by particle-based Molecular Dynamics simulations.

A technique to estimate the height of the energetic barriers for drops in Cassie and Wenzel states is suggested. By this method, we showed that the height of the energetic barrier increases with the strength of solid-liquid interaction $\epsilon_{\mathrm{s}}$, as was initially expected. To overcome an energetic barrier for the drop on a corrugated substrate, one has to apply an external force. We confirmed predictions of lattice Boltzmann simulations [72], where the droplet with the contact angle $\theta_{C} \approx 145^{\circ}$ on a corrugated substrate exhibited parabolic velocity profile at the substrate, $v_{x}(z) \sim z^{2}$, that was followed by the linear one further away from it, $v_{x}(z) \sim z$. However, we also found that for larger contact angle $\theta_{\mathrm{C}} \approx 160^{\circ}$, the velocity profile is well approximated by the linear dependence across the entire height of the droplet, as predicted by scaling arguments [125].

Additionally, we incorporated a chessboard corrugation of the solid with the same ratio $\varphi$ of the area covered by posts to the projected area, as in the case of symmetric grooves. At most of the strengths of solid-liquid interaction $\epsilon_{\mathrm{s}}$, the velocity profiles of droplets pushed by a body force over the chessboard substrate are somewhat higher than the ones of droplets on symmetric grooves, as was shown by Moradi et al. [126]. However, at $\epsilon_{\mathrm{s}}=0.4 \epsilon$, the topography of the substrate did not influence the velocity profile. Therefore, the reduction of the friction is not always possible to reach by modification of substrate topography, as additional friction arises from the edges of the corrugations. 
Then, the flow of a polymer liquid in channels with patterned and flat walls was studied. First, we measured the normal component of the pressure in the bulk at different widths of the channels of different topography and compared the slippage and the friction at the coexistence pressure. Significant differences between flat and corrugated substrates were found for liquids in Cassie state. Owing to the friction at the edges of the grooves, however, the friction coefficient $\lambda$ does not scale like the ratio of area covered by the grooves, $\varphi$, to the projected area. For microscopically corrugated substrates, a correction associated with the fiction at the edges of the corrugations is suggested, c.f. Eq. 4.15.

We showed that there is no sharp phase transition between Cassie and Wenzel states but only a rather gradual crossover. These observations indicate that the macroscopic concepts cannot be straightforwardly extrapolated down to substrate topographies with dimensions that correspond to tens of fluid particle diameters. Using Couette and Poiseuille flow, we extracted the hydrodynamic boundary position, $z_{\mathrm{h}}$, and slip length, $\delta$, that characterize the Navier slip condition. In applications to superhydrophobic substrates of complex topography the position of the hydrodynamic boundary is not intuitive. While the equivalent no-slip plane, which depends on the type of flow, might be located between the top and bottom of the grooves [130], we find that the position of the hydrodynamic boundary, $z_{\mathrm{h}}$, was almost always located above the top of the roughness. 


\section{CHAPTER 5}

\section{ASYMMETRICALLY STRUCTURED SUBSTRATES}

I like pushing boundaries.

Lady Gaga

A possibility to drive a liquid droplet in a controlled way is crucial for micro- and nanofluidics. Directed motion could be used to transport substances suspended in the drop to distinct parts of the lab-on-a-chip or any other microfluidic device.

In order to actuate a droplet, sitting on the substrate, one has to employ surface-energy gradients. These surface-energy gradients may be divided into two general groups [136]: static and dynamic. Good examples of the former ones are methods based on asymmetric dewetting: solids patterned by posts of variable density [136, 137] or chemical gradients [138]. Dynamic gradients, on the other hand, are made by changing the wettability in time, for example, by electrowetting [139] or continuous reactive wetting $[5,140]$. There are actuating approaches using a combination of both, static and dynamic, gradients. The drop on a static substrate can be driven by dynamical temperature gradient [141], driving force [142] or vibrations of the solid [143]. The vibrations in the latter case can be asymmetric or symmetric on asymmetric substrates [144-146]. In this section we study conditions for realization of a directed transport of droplets, sitting on asymmetrically structured and vibrating substrates (ASVS). The response of drops is studied as a function of its size and period of vibrations. To this end, our main concern consists in resolving two questions:

- what is the driving mechanism? A typical mechanism is the one, when a drop is driven by its contact lines (CLs), as the responses of the advancing and receding contact lines on a vibrating asymmetric substrate are not identically rapid [137, 147]. We, on the other side, find a range of vibration periods, leading to the droplets driven additionally by the contact area (CA) of the substrate between the contact lines. In this regime both mechanisms are simultaneously active.

- what is the character of directed motion? In general, there are three possibilities of droplet motion [27, 72]: sliding, rotating and a combination of both. All of them 


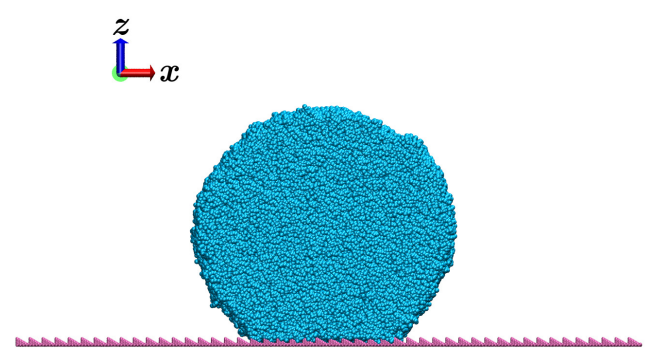

(a)

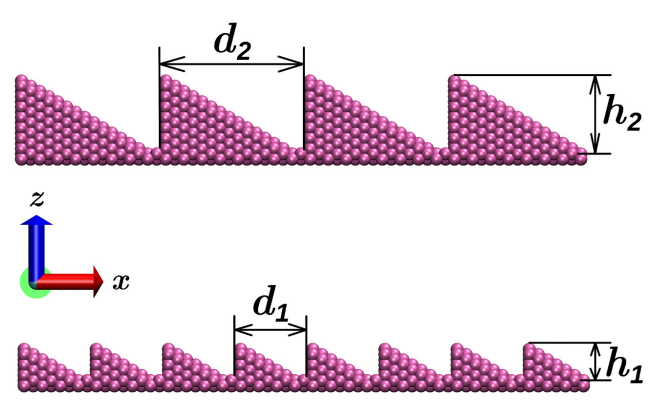

(b)

Figure 5.1: (a) A cylindrical liquid drop on an asymmetrically structured substrate. Side view. (b) The geometries of asymmetrically structured substrates. Two substrates with grooves of different size are studied: roughly corrugated, R (top) and finely corrugated, $\mathrm{F}$ (bottom). The dimensions of the former one are $d_{1}=4.81 \sigma$ and $h_{1}=2.78 \sigma$. The later one is 2 times greater, $d_{2}=9.62 \sigma$ and $h_{2}=5.56 \sigma$. The angle of corrugation is $30^{\circ}$ in both cases.

dissipate the input power by different means. We investigate the character of directed motion as a function of period of substrate vibrations $T_{\text {per, }}$ strength of solid-liquid interaction $\epsilon_{\mathrm{s}}$ and corrugation of the substrate (F- or R-type). We find that most of the droplets are sliding, but the corrugation may give a rise to additional rotation.

This chapter is organized as follows: at first, in section 5.1.1, we present the topography of the substrate and dimensions of the system. Then, in section 5.1.2, we explain the response of a droplet onto agitation and the direction of motion. The results on mechanisms of directed motion are reported in section 5.1.3. The section 5.1.4 deals with character of directed motion, manners of dissipations and efficiency of directed motion. We conclude with a short discussion and give an outlook in section 5.2.

\subsection{Directed transport of drops on agitated superhydrophobic substrates}

\subsubsection{Agitated systems}

Here, we present details about the simulation set-up and the topography of ASVSs. Additionally, we discuss the agitation process and its parameters.

At first, we note, that a polymer droplet is modeled in three dimensional simulation domain of volume $V=L_{x} L_{y} L_{z}$. Periodic boundary conditions are used in $x$ - and $y$ directions, whereas an ideal repulsive wall is placed far above the droplet, sitting on a supporting substrate, in $z$-direction. The lengths of the simulation box are chosen in a way to provide the formation of cylindrical droplets, namely $L_{y}=30.25 \sigma \ll L_{x}$. 


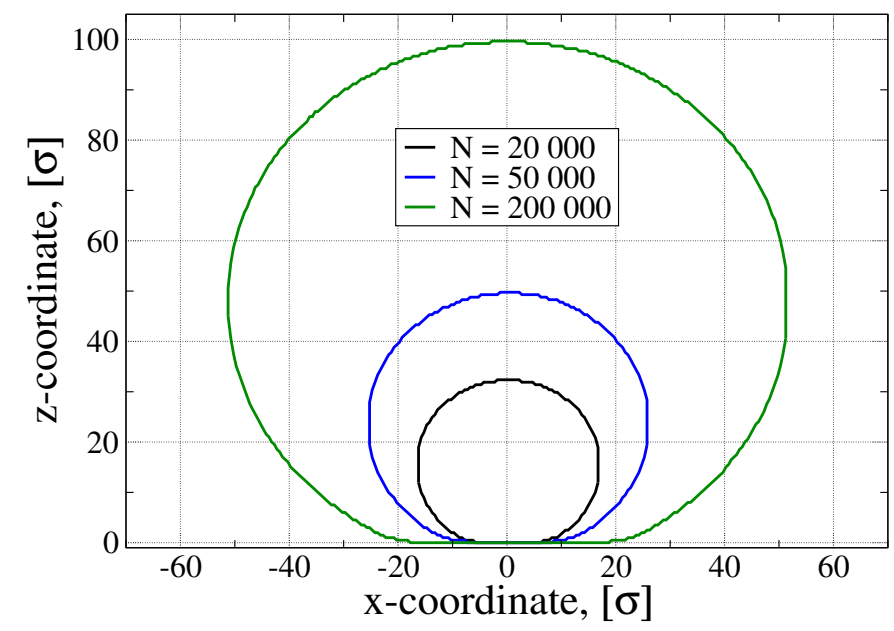

Figure 5.2: The profiles of drops of varying size on F-type substrate at $\epsilon_{\mathrm{S}}=0.4 \epsilon$ and period of substrate vibration $T_{\text {per }}=41 \tau$.

The side view of a system is shown in Fig 5.1a, the axis of a drop is parallel to $y$-axis of the box. We remind that due to cylindrical geometry, one may (i) study drops of bigger radii than in the case of spherical droplets and (ii) neglect line tension effects as explained in Section 3.2.

Then, we limit us to two substrates of fine (F-type) and rough (R-type) corrugations. The grooves of both substrates have triangular shape in side view as shown in Fig. 5.1b, but differ in size by a factor of 2 . The largest cathetus $d_{i}$ forms a $30^{\circ}$ angle with the hypotenuse.

Finally, we set the drop in motion by starting in-phase oscillations of the substrate atoms. Their velocity is given by the harmonic law

$$
\overrightarrow{v^{\mathrm{s}}}(t)=v_{z}^{\mathrm{s}}(t) \vec{n}=A \vec{n} \omega \sin \left(\omega\left[t-t_{0}\right]\right),
$$

where $A, \omega$ and $t_{0}$ are the amplitude, frequency and the time when agitation starts, correspondingly. $\vec{n}$ is the unit vector normal to the bottom layer of the substrate. The initial velocity $v_{z}^{\mathrm{s}}\left(t_{0}\right)$ is zero and therefore there is no sudden energy and momentum infusion into the system. This facilitates the equilibration phase, because the drops do not detach from the substrate at the moment when the vibrations start.

We investigate the response of the droplet on agitation at interaction strengths $\epsilon_{\mathrm{s}}=$ 0.4 and $0.5 \epsilon$ and at the periods of vibrations $15 \tau \leqslant T_{\text {per }}=2 \pi / \omega \leqslant 251 \tau$. The contact angles of the drops on a flat substrate for these values of $\epsilon_{\mathrm{S}}$ are $138.1^{\circ}$ and $120.8^{\circ}$ upon increasing $\epsilon_{\mathrm{s}}$, respectively. Also, droplets of different size are modeled to address the question of its influence onto the mechanism and efficiency of directed motion. The drops consist of 20000, 50000 or 200000 beads and Fig. 5.2 displays the drops of varying size at $T_{\text {per }}=41 \tau$ on F-type substrate at $\epsilon_{\mathrm{s}}=0.4 \epsilon$. Note, that not only the radii of the droplets differ, but also the contact areas. The contact angles, however, remain equal. 


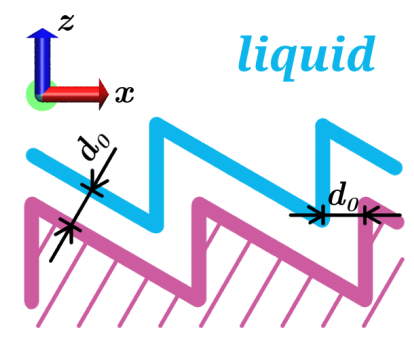

(a)

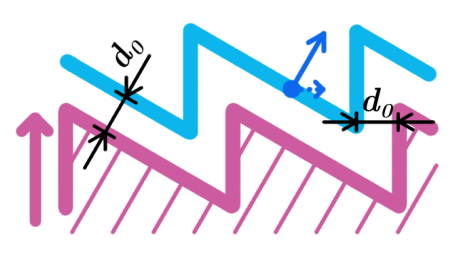

(b)

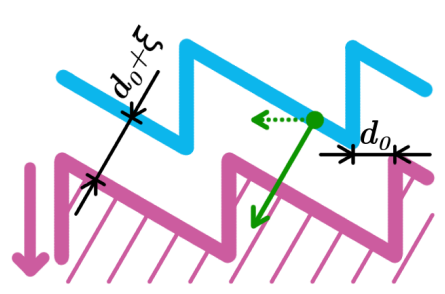

(c)

Figure 5.3: Explanation of the direction of motion: (a) The solid-liquid interface (blue contour) of the droplet resting on a asymmetrically structured substrate (lila contour). The distance from the interface to the solid is $d_{0}$. (b) Raising of the ASVS is marked by the lila arrow. The liquid is pushed by the solid (blue arrow), and the repulsion drives the droplet to the right (blue pointed arrow). Due to steep repulsive part of the LJ potential, the distance from the solid to the interface changes insignificantly. (c) Lowering of the ASVS. The distance from the solid changes by amount of $\xi$, shifting the effective interaction into attractional range of the potential (green arrow). It has a component directed to the left (green pointed arrow) that on average is greater than repulsive one and provides the directed motion to the left.

\subsubsection{Response of a drop on agitation}

In this section we explain the direction of motion by simple analytical considerations. Then the hypothesis is supported by computer simulations. Later, the shape of the droplets and the parameters that affect it are discussed.

A droplet placed on a resting asymmetrically structured substrate can not move in any specific direction (Fig. 5.3a), as it would act like a Maxwell's demon[148] taking advantage of thermal fluctuations and violating the second law of thermodynamics. However, if one constantly inputs energy into the system, e.g. by vibration of the substrate, the droplet can be moved in a definite direction in a controlled way. The direction itself can be justified by the following considerations.

A naive thought would be that during the raise of the substrate from its minimal position to the maximal one the liquid is pushed into direction normal to the one of the grooves, as shown in Fig. 5.3b. The force the substrate exerts on a drop $\overrightarrow{F^{\mathrm{s}}}(t)$ (blue solid arrow) depends on time $t$ and has a component $F_{\text {rais }}(t) \vec{i}$ (blue pointed arrow), pointing to the right side, where $\vec{i}$ is a unit vector in $x$ direction.

We expect that the drop would indeed go in this direction if the solid-liquid interaction is purely repulsive. However, we use the LJ potential that has an attractive part (Eq. 2.5). Therefore, the other stage of substrate movement should also be considered, namely the one with the solid moving downwards as in Fig. 5.3c. At this stage the solid-liquid distance increases by amount of $\xi$, and atoms of the substrate attract the 


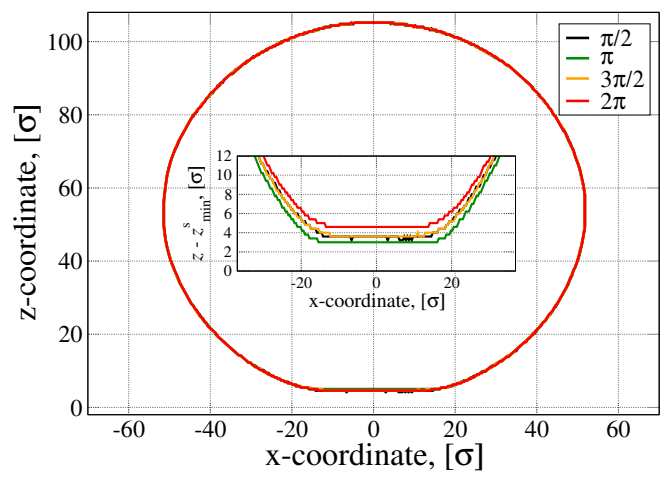

(a)

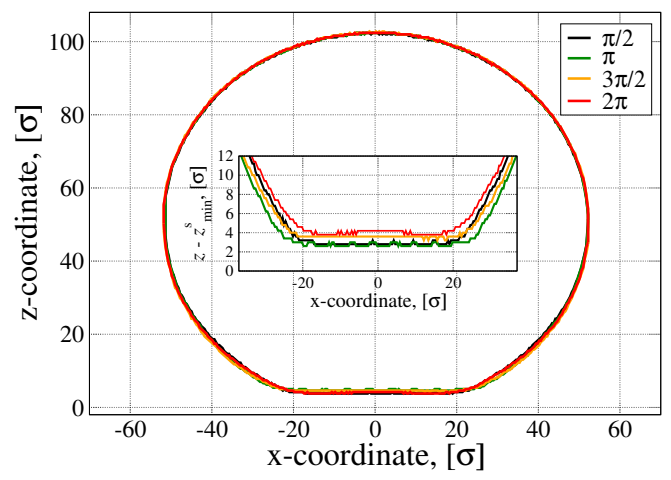

(b)

Figure 5.4: (a) and (b) show profiles of drops of $N=200000$ beads averaged at the same phase $\omega\left[t-t_{0}\right]$ of oscillations on F-type substrate at $\epsilon_{\mathrm{s}}=0.4 \epsilon$ with $T_{\text {per }}=15 \tau$ and $63 \tau$ vibrating periods, correspondingly. Insets zoom into the region close to the substrate and show the distance from the solid-liquid interface to the bottommost layer of the substrate $z_{\min }^{\mathrm{s}}$.

liquid (green solid arrow). This attraction pulls the liquid to the left with the force $F_{\text {lower }}(t) \vec{i}$ (green pointed arrow) that act against the force $F_{\text {rais }}(t) \vec{i}$. Now, taking into account steep repulsive part of the LJ potential and relatively gentle slope of attractive part, we conclude that the average position of the solid-liquid interface is shifted towards larger distances with respect to the minimum of the potential. This explains, that effectively the droplet is driven by substrate vibrations to the left.

We justify our considerations in three steps:

- considering the biggest droplet profiles averaged at specific phases of substrate vibrations with periods $T_{\text {per }}=15 \tau$ and $63 \tau$ in Fig. 5.4a and 5.4b, respectively. In particular, we look how the distance from solid-liquid interface to the bottommost substrate atom plane, $z_{\text {min }}^{\mathrm{s}}$, depends on the phase (the insets). Indeed, as was suggested, the solid-liquid interface is located farther away from the solid when the substrate is moving down (phases $3 \pi / 2$ and $2 \pi$, orange and red lines) then when raising up (phases $\pi / 2$ and $\pi$, black and green lines);

- considering the velocity fields of the biggest droplets averaged at specific phases of substrate vibrations with the same periods as in previous step. The velocity fields are shown in Fig. 5.5 and 5.6 for $T_{\text {per }}=15 \tau$ and $T_{\text {per }}=63 \tau$, respectively. In particular, we look how the particles of the liquid are moving in the vicinity of the solid. When raising the substrate, the boundary layer of the liquid is pushed upwards and mostly to the right, as expected. However, the strength of the phenomena depends on the period and phase. For example, the phase $\pi$, when the substrate reaches its maximum position has very symmetric velocity field for all the periods. This is explained by the fact that the compression of the liquid at 


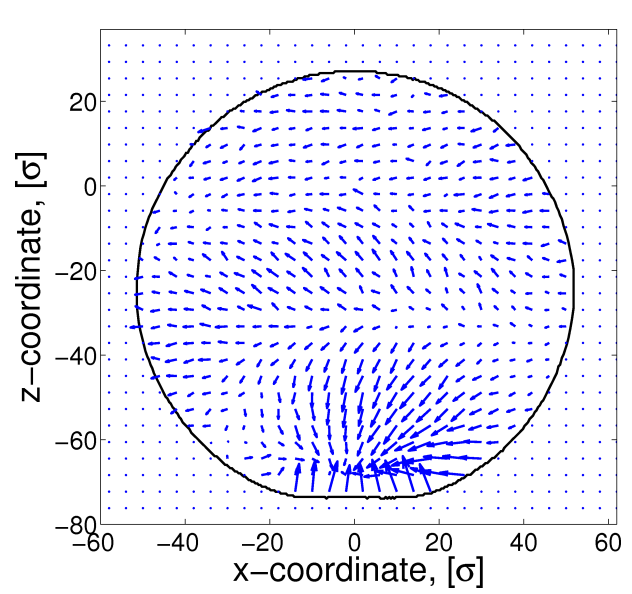

(a)

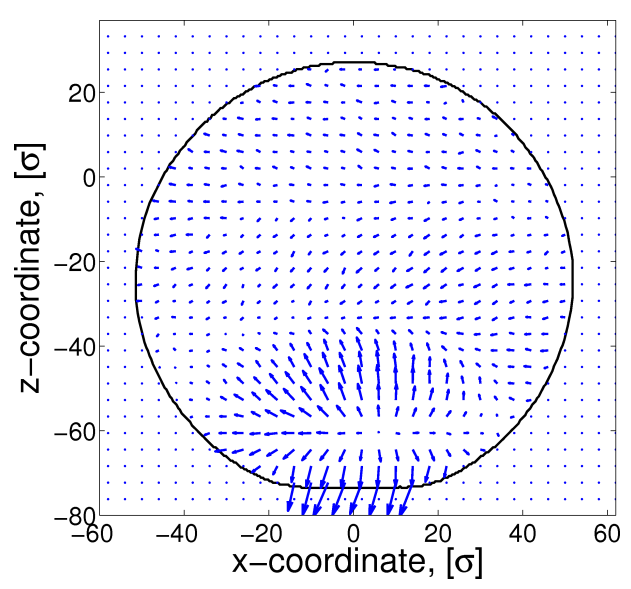

(c)

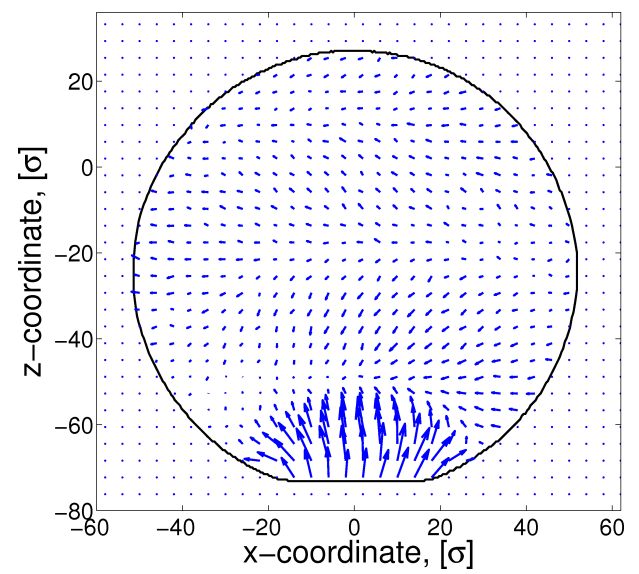

(b)

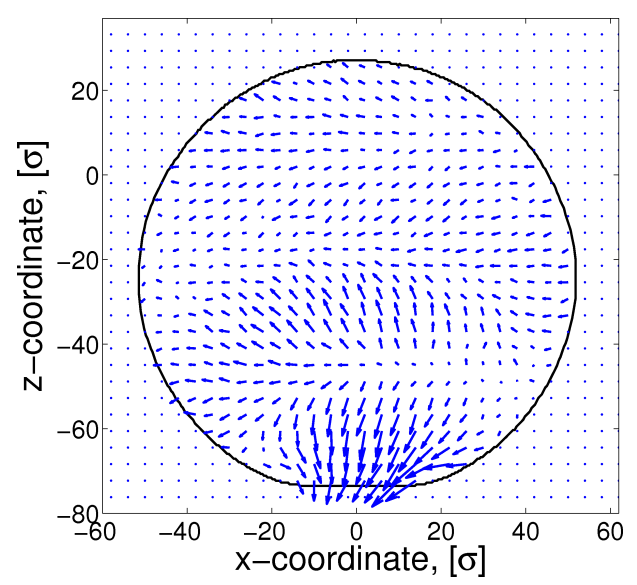

(d)

Figure 5.5: Velocity fields of droplets of $N=200000$ beads averaged at the same phase $\omega\left[t-t_{0}\right]$ of the F-type substrate vibrations with period $T_{\text {per }}=15 \tau$ and at solid-liquid interaction strength $\epsilon_{\mathrm{s}}=0.4 \epsilon$. (a) and (b) correspond to the raising of the substrate at phases $\pi / 2$ and $\pi$, respectively. (c) and (d) correspond to the lowering of the substrate at phases $3 \pi / 2$ and $2 \pi$, respectively. The corrugations of the substrate are not spatially resolved on the profiles at these strength of the solid-liquid interaction and period of vibrations.

this stage of substrate vibrations is the highest and the liquid's response is nearly isotropic.

When the substrate is going down and starts to attract liquid the picture is different. We observe mostly the breaking of the symmetry of the velocity field with horizontal net component of the velocity, pointing to the left. It is also interesting to notice that the propagation of the flux created by the substrate vibrations is different for two periods. If we consider the positions of the substrate at its maximum and minimum ( $\pi$ and $2 \pi$, correspondingly), the substrate sets in motion the whole bottom half of the droplet at higher period (cf. 5.6b and 5.6d), whereas for a smaller one, considerably smaller part of the droplet is involved (cf. 5.5b 


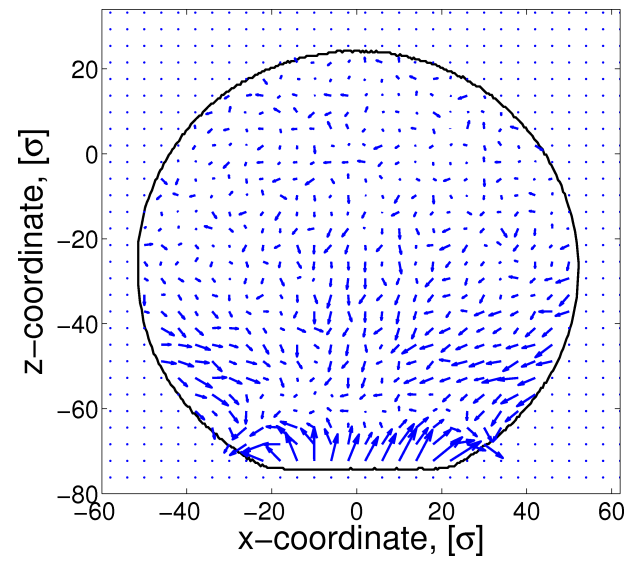

(a)

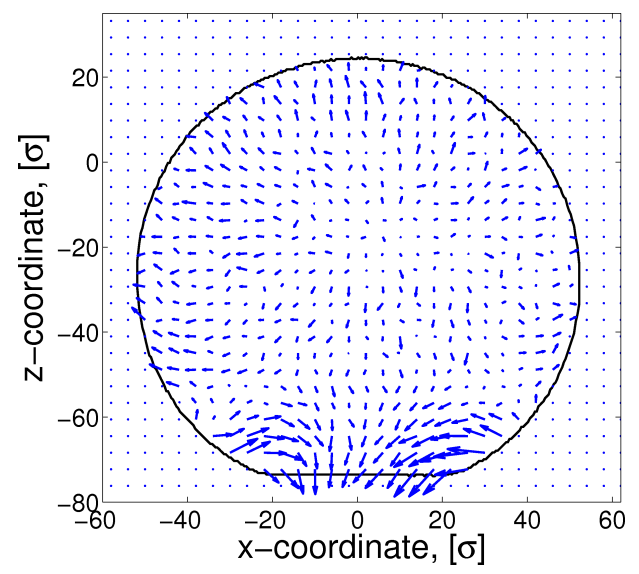

(c)

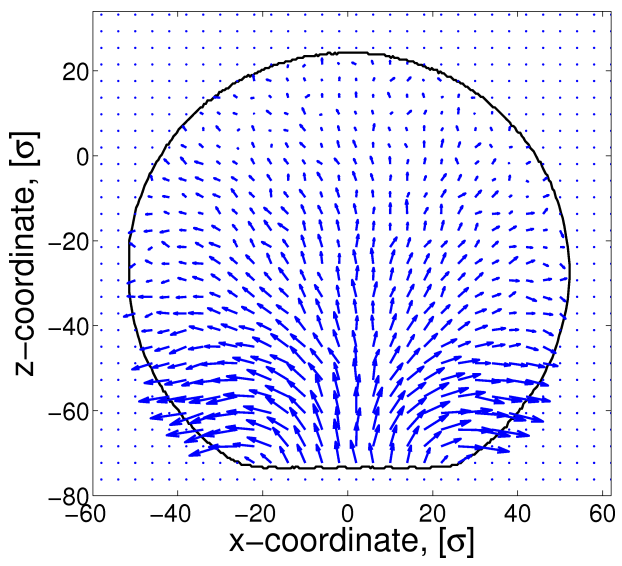

(b)

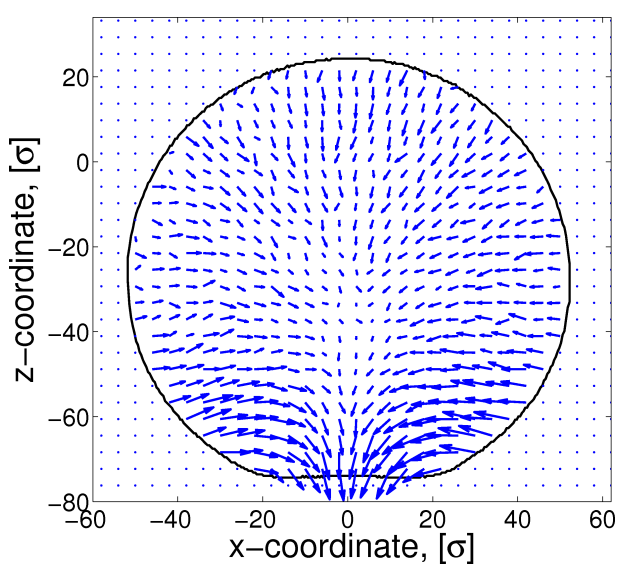

(d)

Figure 5.6: Velocity fields of droplets of $N=200000$ beads averaged at the same phase $\omega\left[t-t_{0}\right]$ of the F-type substrate vibrations with period $T_{\text {per }}=63 \tau$ and at solid-liquid interaction strengths $\epsilon_{\mathrm{s}}=0.4 \epsilon$. (a) and (b) correspond to the raising of the substrate at phases $\pi / 2$ and $\pi$, respectively. (c) and (d) correspond to the lowering of the substrate at phases $3 \pi / 2$ and $2 \pi$, respectively.

and 5.5d). It may also be explained by a simple suggestion that at higher period, the influence of the substrate has more time to propagate into the liquid;

- calculating the average forces $\overline{F_{\text {rais }}}$ and $\overline{F_{\text {lower }}}$, acting on the biggest droplet when raising (phase of vibrations from 0 to $\pi$ ) and lowering (phase from $\pi$ to $2 \pi$ ) the F-type substrate, respectively. These forces are quantified by

$$
\overline{F_{\text {rais }}}=\left\langle\frac{1}{T_{\text {per }}} \int_{0}^{\pi} F_{\text {rais }}(t) d t\right\rangle=\left\langle\frac{1}{T_{\text {per }}} \int_{0}^{\pi} \overrightarrow{F^{\mathrm{s}}}(t) \cdot \vec{i} d t\right\rangle,
$$

and

$$
\overline{F_{\text {lower }}}=\left\langle\frac{1}{T_{\text {per }}} \int_{\pi}^{2 \pi} F_{\text {lower }}(t) d t\right\rangle=\left\langle\frac{1}{T_{\text {per }}} \int_{\pi}^{2 \pi} \overrightarrow{F^{\mathrm{s}}}(t) \cdot \vec{i} d t\right\rangle,
$$

where $\overrightarrow{F^{\mathrm{s}}}$ is the force the substrate exerts on a drop. The angle brackets $\langle\ldots\rangle$ stand for ensemble averages. The results are brought together in Table 5.1. The 


\begin{tabular}{c|c|c}
$T_{\text {per }}$ & $\overline{F_{\text {rais }}},\left[\sigma / \tau^{2}\right]$ & $\overline{F_{\text {lower }}},\left[\sigma / \tau^{2}\right]$ \\
\hline $15 \tau$ & $12.8 \pm 3$ & $-16.0 \pm 2.9$ \\
$63 \tau$ & $1.1 \pm 4.5$ & $-11.9 \pm 4.2$
\end{tabular}

Table 5.1: The average forces $\overline{F_{\text {rais }}}$ and $\overline{F_{\text {lower }}}$, acting on a droplet during raising and lowering F-type substrate at period $T_{\text {per, }}$, respectively. The average net force has a negative sign and therefore point to the left, driving droplet in this direction.

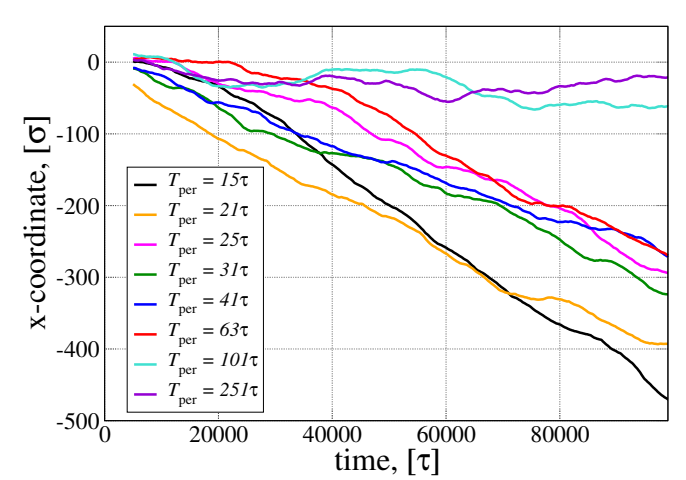

(a)

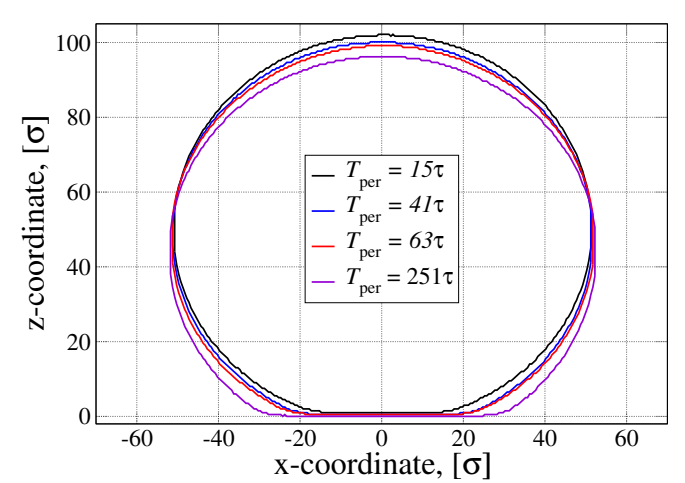

(b)

Figure 5.7: (a) The position of the center of mass of the drop of $N=200000$ monomers at $\epsilon_{\mathrm{s}}=0.4 \epsilon$ during the simulation at different periods of substrate vibrations $T_{\text {per }}$. (b) The profiles of drops of $N=200000$ beads on F-type substrate at interaction strength $\epsilon_{\mathrm{s}}=0.4 \epsilon$. Different colors represent profiles at various periods of substrate oscillation.

average net force has a negative sign and therefore point to the left, driving the droplet in this direction.

However, although the net force at moderate period of vibrations $T_{\text {per }}=63 \tau$ is greater then the one at $T_{\mathrm{per}}=15 \tau$, this does not necessarily indicate that the velocity of the center of mass of the drop $(\mathrm{CM}), V_{\mathrm{CM}}$, will be higher in the first case. The reason is that not all the energy provided by the substrate is converted into translational motion of the droplet, but part of it is dissipated by viscous and frictional forces. The detailed study of the efficiency of driving is given in Sec. 5.1.3.

After a short time after the start of substrate vibrations, the drop establishes a steadystate motion regime. In Fig. 5.7a we report on the coordinate of the CM of the droplet during the simulation. Different colors represent different periods of substrate vibrations $T_{\text {per }}$ and nearly constant slope of the curves indicates established steady states.

The last detail connected to the response of the droplet is the change of the contact angle as a function of period of vibrations, $T_{\text {per, }}$, as shown in Fig. $5.7 \mathrm{~b}$. It may be explained by the fact, that the balance of surface tensions at the CL is influenced by the oscillating substrate. The smaller the period, the greater the change of the contact angle (and the shape) of the drop with respect to the one on a substrate at rest, that can be regarded in the limit of high periods (e.g. at $T_{\text {per }}=251 \tau$ ). 

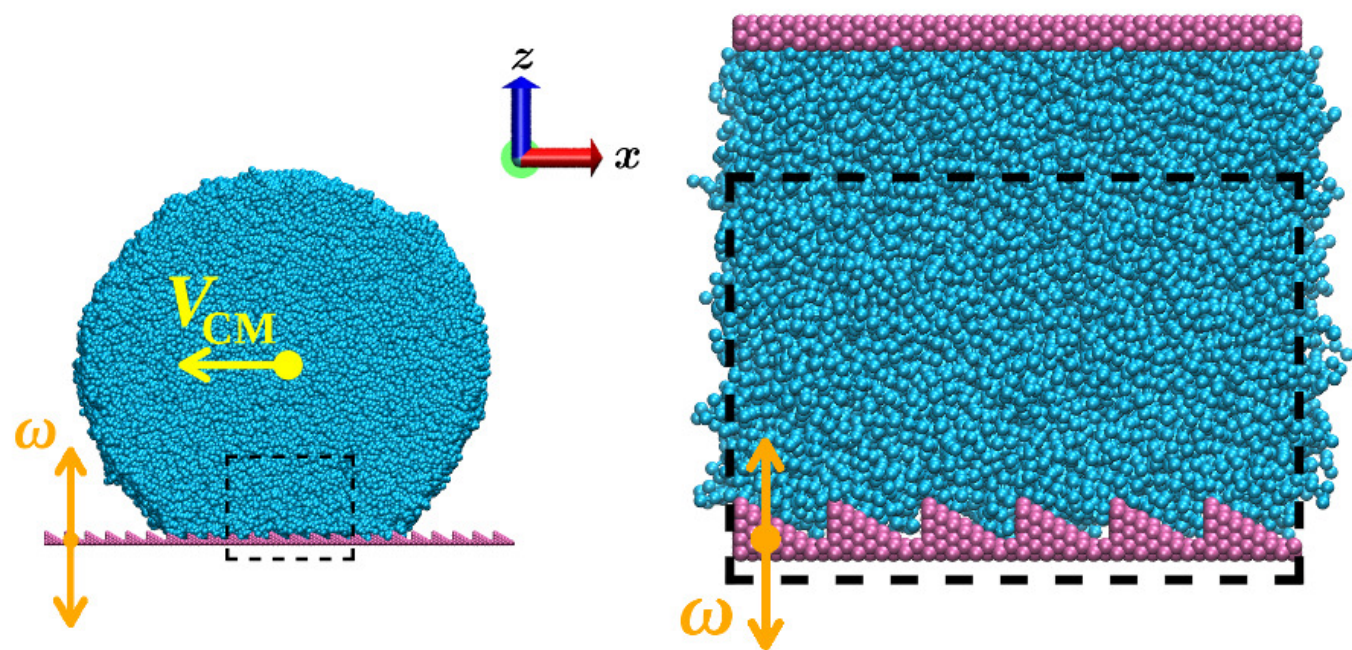

Figure 5.8: Shown are side views of (a) a droplet on the ASVS of F-type and (b) a flow of the liquid between a flat wall and the ASVS of F-type. The dashed regions are equivalent, if the width of the channel is big enough to avoid an influence of the flat wall onto liquid's structure in the dashed box. The lack of advancing and receding CLs allows to study the flows induced by the CA of the liquid and the substrate. If a flow in such system exists, the CA with ASVS will drive the drop together with the CLs. If the flow is missing, the CLs will drive the droplet alone.

\subsubsection{Mechanisms of droplet motion}

In order to sort out the driving mechanisms of droplets on ASVS, we study a liquid volume close to its CA with the solid, corresponding to the dashed box as shown in Fig. 5.8 (left). In $z$ direction, the liquid is confined by a flat wall as displayed in Fig. 5.8 (right), whereas in $x$ and $y$ the periodic boundary conditions are applied. The dashed regions are equivalent, if the packing of the liquid in the vicinity of a flat wall does not influence the dashed box, and the distance between the substrates provides the coexistence pressure in the bulk of the liquid. The density of the vapor phase in pockets of the ASVS is negligible and the coexistence pressure $p_{\text {coex }} \approx 0$. The virtue of a system with a liquid confined between the flat and the ASVS consists in the absence of advancing and receding three-phase CLs. The vibrations of the asymmetric substrate in the channel are set up in the same way as for droplets, as discussed above. We measure the velocity profiles of the liquid along the line connecting two substrates (normal to the flat substrate) at different periods of oscillations $T_{\text {per }}$.

The profiles presented in Fig. 5.9 show that liquid can be set in motion without receding and advancing CLs. Therefore, the droplets on ASVS at short periods of oscillations $T_{\text {per }}$ can be driven by the CA. However, it does not necessarily imply that the CLs are not participating in motion at all, but only that the area of contact with the substrate is an active mechanism of driving in this regime. Therefore, we refer to the situation as the directed motion driven by both, CA and CLs. On the other side, at higher periods there 


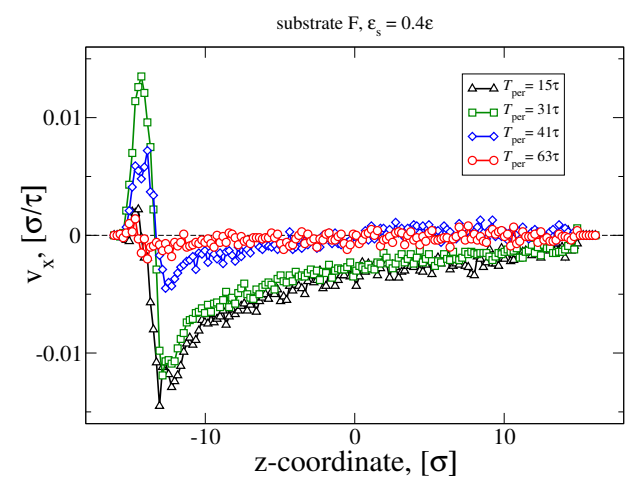

(a)

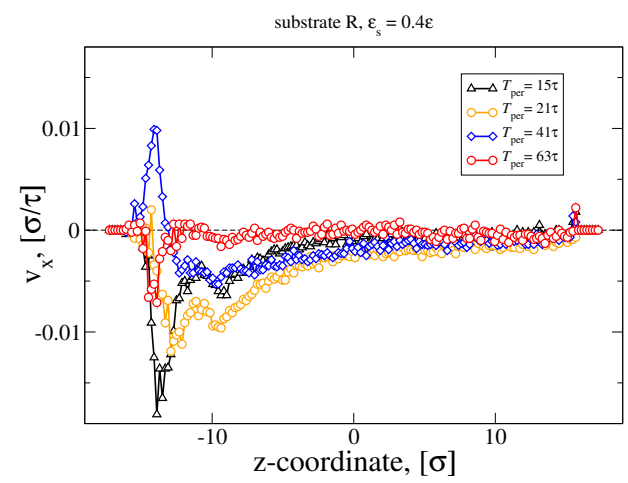

(c)

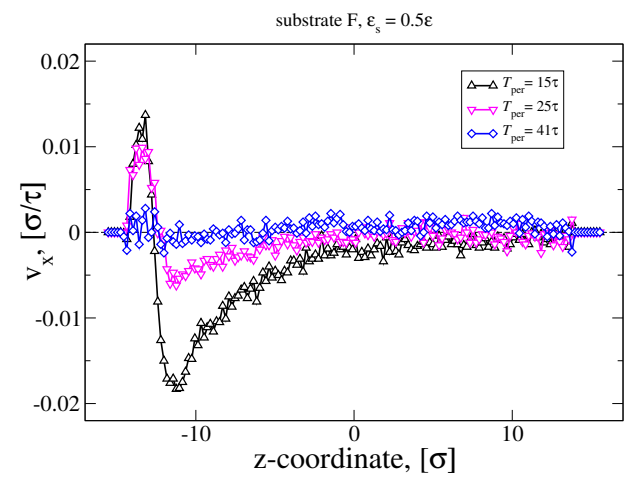

(b)

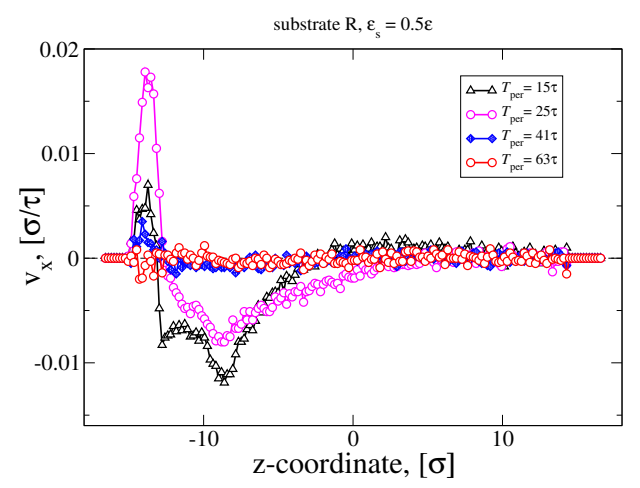

(d)

Figure 5.9: Velocity profiles of the liquid confined between a flat and F-type ASVS at $\epsilon_{\mathrm{S}}=$ $0.4 \epsilon$ (a) and $\epsilon_{\mathrm{s}}=0.5 \epsilon$ (b). The liquid is set in motion for $T_{\text {per }} \leqslant 41 \tau$ and $T_{\text {per }} \leqslant 25 \tau$, for (a) and (b), respectively. The same for a flat and R-type ASVS at $\epsilon_{\mathrm{s}}=0.4 \epsilon$ (c) and $\epsilon_{\mathrm{s}}=0.5 \epsilon$ (d). The liquid is set in motion for periods of oscillations upto $T_{\text {per }} \leqslant 63 \tau$ and $T_{\text {per }} \leqslant 41 \tau$, for (c) and (d), respectively.

is no driving by the CA. Drops are set in motion due to the advancing and receding CLs only. This regime starts for the F-type ASVS at shorter periods than for the R-type ASVS, namely $T_{\text {per }}>41 \tau$ and $T_{\text {per }}>25 \tau$ for the F-type ASVS, and $T_{\text {per }}>63 \tau$ and $T_{\text {per }}>41 \tau$ for the R-type ASVS at $\epsilon_{\mathrm{s}}=0.4 \epsilon$ and $\epsilon_{\mathrm{s}}=0.5 \epsilon$, respectively.

Returning back to the case of droplets on ASVSs, we report in Fig. 5.10 on the velocity of the droplet's $\mathrm{CM}, V_{\mathrm{CM}}$, as a function of period of vibrations $T_{\text {per }}$ and solid-liquid interaction strength $\epsilon_{\mathrm{s}}=0.4 \epsilon$ at F-type substrate. The inset stands for same parameters, but at $\epsilon_{\mathrm{S}}=0.5 \epsilon$. The behavior of $V_{\mathrm{CM}}$ correlates with the mechanisms of driving.

When the CA and CLs are active simultaneously (shaded region), the change of droplet's size significantly affects the CM velocity. Here we note that the length of the CLs, $2 L_{y}$, is independent of the size, and that the CA, $A_{\text {drop }}$, is proportional to the radius of the drop, $R$, and the sinus of contact angle, $\sin \alpha$. Therefore, in this regime for drops of varying size one sees scaling of the $V_{\mathrm{CM}}$ with a factor of the CA, $A_{\mathrm{drop}}$. The other regime is the one when only CLs are active. Since the length of the CLs is independent 


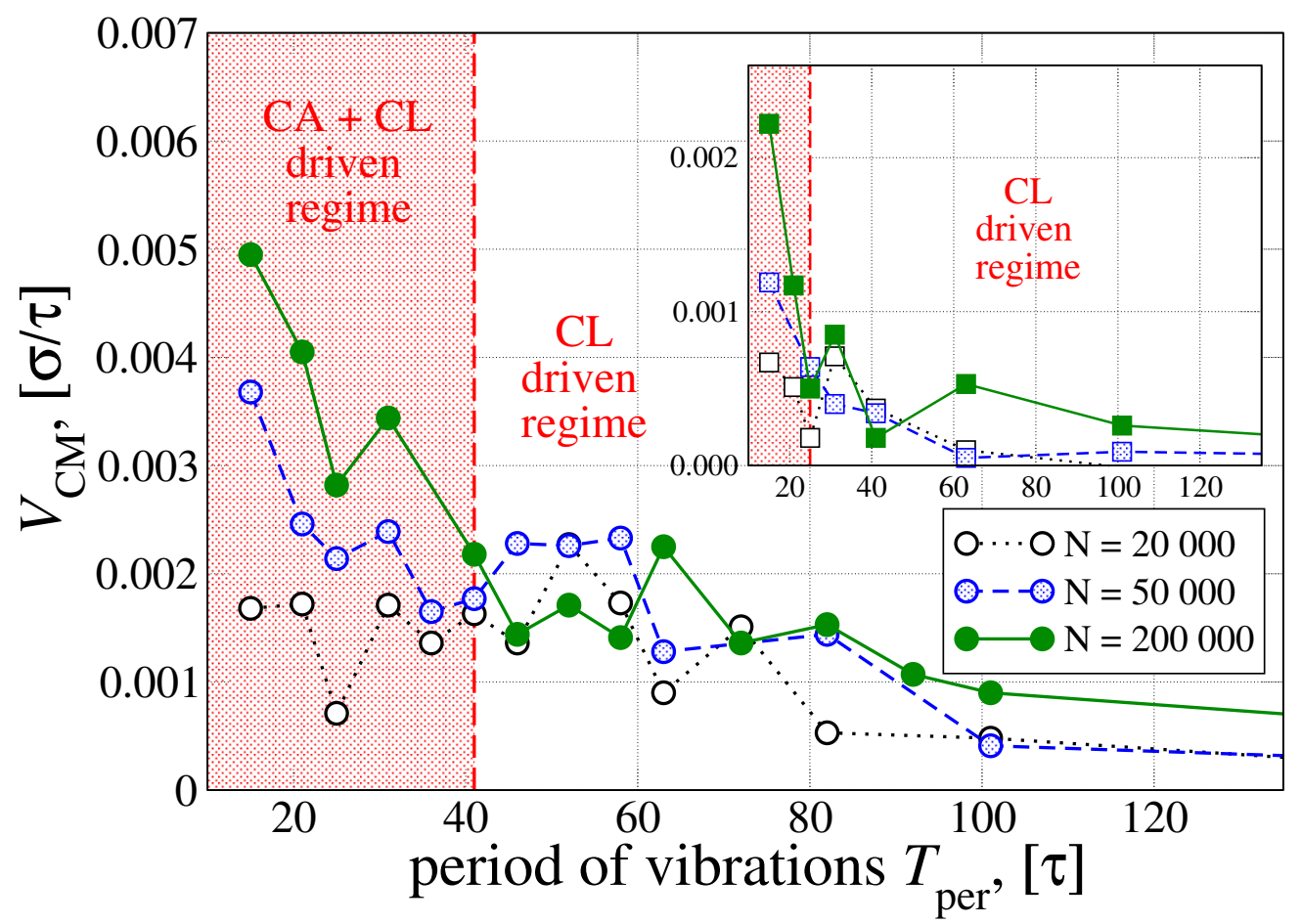

Figure 5.10: Velocity of the $\mathrm{CM}, V_{\mathrm{CM}}$, for drops of different sizes as a function of period of F-type substrate vibrations at $\epsilon_{\mathrm{S}}=0.4 \epsilon$. The inset displays the same dependence at $\epsilon_{\mathrm{S}}=0.5 \epsilon$.

of droplet's size, there is no more any considerable difference of the $V_{\mathrm{CM}}$ for different droplets.

To conclude, we emphasize that there are two possible mechanisms of driving: (i) at short periods of substrate vibrations the drop is driven by both, the area of contact with the substrate and three-phase contact lines; (ii) upon increasing the period the driving due to the CA looses its power and at higher periods the drop is driven solely by CLs. The investigation of the efficiency of the driving follows in the next section.

\subsubsection{Character of motion. On the way to sort out the dissipations}

This section is devoted to the investigation of the character of directed motion (whether sliding, rotational one or combination of both) and estimate the dissipations during the motion. At first, we quantify the power input provided by the substrate. Later, the different manners this power is dissipated are reviewed. In the end, we explain how the droplet is moving and compare character of motion as a function of period of substrate vibrations $T_{\text {per }}$, strength of solid-liquid interaction $\epsilon_{\mathrm{s}}$ and corrugation of the substrate.

The droplet placed onto the ASVS is reacting onto vibrations: it adjusts its shape and starts to move in a certain direction. This motion is always supported by the substrate 


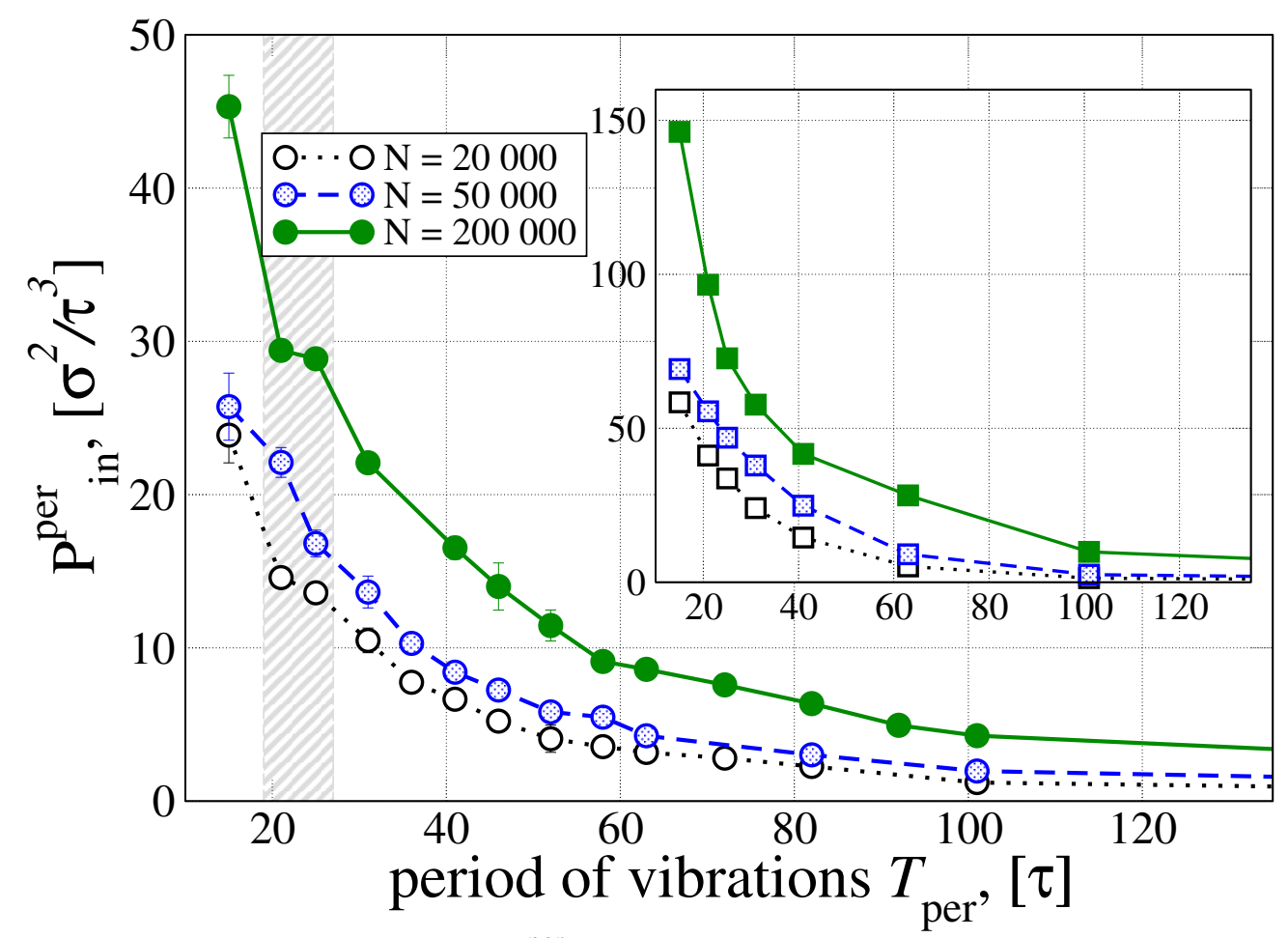

Figure 5.11: The input power $P_{\text {in }}^{\text {per }}$ during one period $T_{\text {per }}$ of vibration of F-type substrate at $\epsilon_{\mathrm{s}}=0.4 \epsilon$ and as a function of the size of the drop. The common trend is the decrease of the power with the period of oscillations. However, one can notice peculiar behavior in the region of $20-25 \tau$. See main text for details. The inset presents the input power per period at $\epsilon_{\mathrm{S}}=0.5 \epsilon$ for drops of different size.

vibrations, but not all the power introduced by the ASVS is spent onto translational motion. Substantial part of it is dissipated by different means. The starting point on the way to estimate the efficiency of directed motion of the droplet on the ASVS is the knowledge of the power input, $P_{\text {in }}^{\text {per }}$, provided during a single period of substrate oscillations.

The power input to the droplet on the ASVS provided during a single period of substrate oscillations can be written as

$$
P_{\text {in }}^{\text {per }}=\frac{1}{T_{\text {per }}} \int_{0}^{T_{\text {per }}} v_{z}^{\mathrm{s}}(t) \vec{F} \mathrm{~s}(t) \cdot \vec{n} d t
$$

The velocity of the substrate, $v_{z}^{\mathrm{s}}(t)$, decreases with period of oscillations, and therefore we expect that the input power is also decreasing function of $T_{\text {per. }}$. Indeed, one finds this type of behavior in Fig. 5.11. The power input of a single vibration, $P_{\text {in }}^{\text {per }}$, is shown there for drops of varying size at $\epsilon_{\mathrm{s}}=0.4 \epsilon$ as a function of the period of vibrations, $T_{\text {per }}$. The inset shows the same data at $\epsilon_{\mathrm{s}}=0.5 \epsilon$. In general, the dependences are monotonic for drops of a fixed size. It is natural to suggest that the amount of input power is proportional to the CA of the droplet, $A_{\text {drop }} \sim R \sin \alpha$, i.e. $P_{\text {in }}^{\text {per }} \sim R \sin \alpha$.

The common feature for drops of all sizes are the peculiarities in the region of $20-25 \tau$ 
(shaded area in Fig. 5.11). To investigate the reason behind it, one should look at the balance of dissipations in the system.

Following the notation introduced by de Gennes [80], the total dissipation during droplet motion, $T \Sigma$, is the sum of the viscous dissipations, $T \Sigma_{w}$, frictional dissipations at the $\mathrm{CA}, T \Sigma_{A}$, dissipations at the three-phase CLs, $T \Sigma_{l}$, and dissipations in the precursor film, $T \Sigma_{f}[27,125]$ :

$$
T \Sigma=T \Sigma_{w}+T \Sigma_{A}+T \Sigma_{l}+T \Sigma_{f}
$$

The last term in our system is zero, as we do not have any precursor film, but the other types of dissipations reduce the amount of the power input transformed into directed motion:

$$
V_{\mathrm{CM}}^{2} \sim P_{\mathrm{in}}^{\mathrm{per}}-T \Sigma_{w}-T \Sigma_{A}-T \Sigma_{l}
$$

Now, let us consider the origin of individual terms of Eq. 5.6. The viscous dissipation in the droplet with velocity field $\vec{u}$ is defined as

$$
T \Sigma_{w}=\eta \int_{V_{\mathrm{diss}}}(\nabla \vec{u})^{2} d V_{\mathrm{diss}}
$$

where $V_{\text {diss }}$ is the volume over which the viscous dissipations occur. The third term on r.h.s. of Eq. 5.6 stems from the fact that the liquid slips over the solid that results in friction. The last type of dissipations, that can take place in the studied system, is the one related to dissipations at the CLs. The origin of this dissipation lies in: (i) elastic deformations of the CL itself [149] and (ii) displacements of liquid beads at the CLs [150]. The displacements here are related to the change of the positions in time and are disconnected to the solid displacements.

To sort out the character of droplet motion and associated dissipation mechanisms we plot velocity field diagrams of droplets at the ASVS of F-type in Table 5.2. The velocity fields are measured with respect to the CM of the droplet, but its velocity is not subtracted. Shown are the largest droplets of $N=200000$ beads, however, the behavior of the smaller ones is similar. Top row corresponds to solid-liquid strength $\epsilon_{\mathrm{s}}=0.4 \epsilon$, bottom one to the $\epsilon_{\mathrm{s}}=0.5 \epsilon$. Only two periods of vibrations are plotted, namely $T_{\text {per }}=15 \tau$ and $63 \tau$ (left and right columns, correspondingly). The choice of the periods is dictated by the driving mechanisms: for smaller one it is CA plus CLs, for larger one it is only CLs that drive the drop. The other periods corresponding to the one or the other mechanism of driving are similar to the ones that are shown. The only difference consists in the strength of the effect.

For all the periods of vibrations at $\epsilon_{\mathrm{s}}=0.4 \epsilon$ (top row in Table 5.2) the drop slides to the left without any significant steady-state rotation. However, in the vicinity of the receding CL (right side) there is always a flux in clockwise direction. This effect is more pronounced if one increases the strength of solid-liquid interaction to $\epsilon_{\mathrm{s}}=0.5$ (bottom 


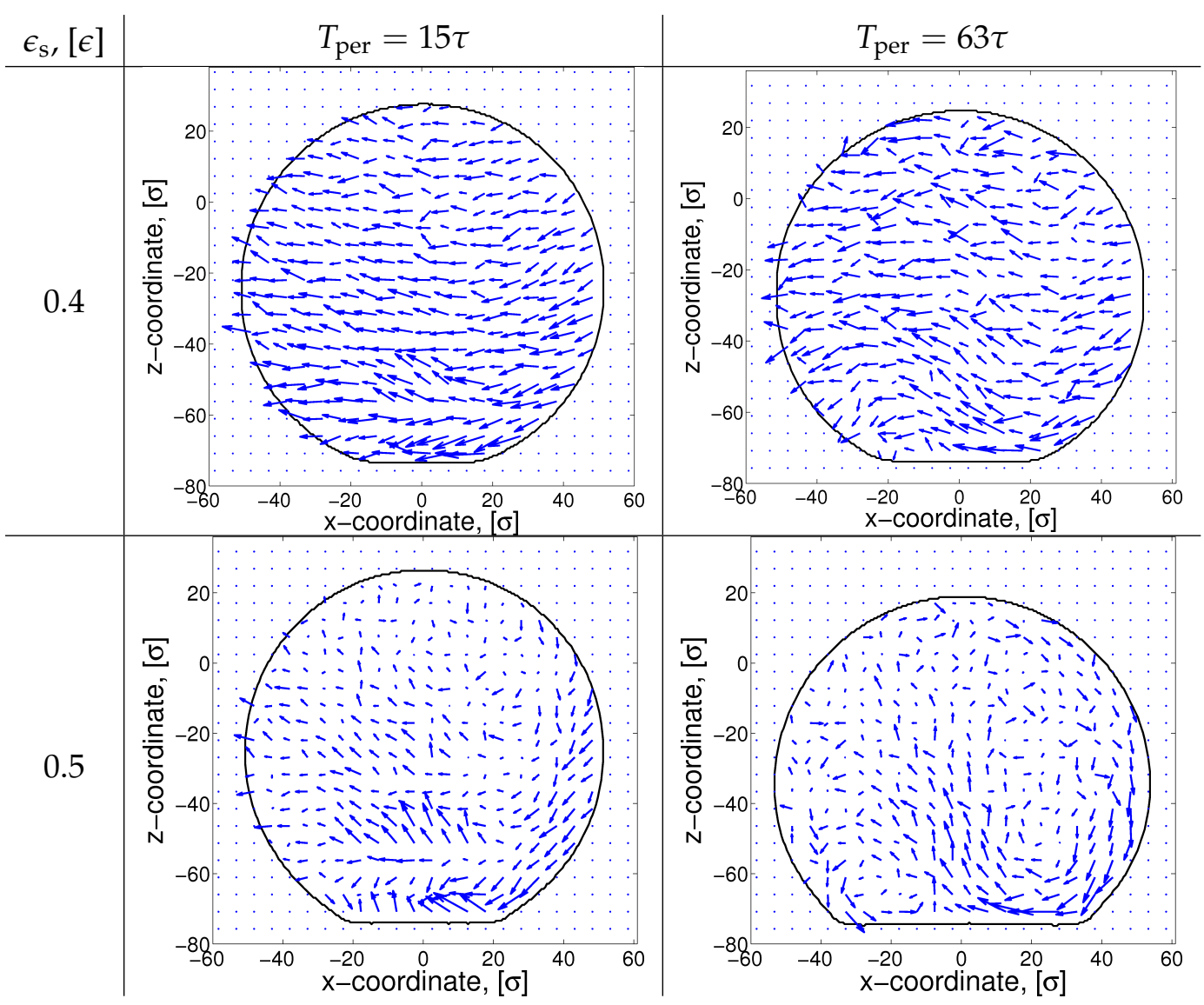

Table 5.2: Velocity fields of moving droplets of $N=200000$ beads at the F-type substrate with solid-liquid interaction strengths $\epsilon_{\mathrm{s}}=0.4 \epsilon$ (top row) and $\epsilon_{\mathrm{s}}=0.5 \epsilon$ (bottom row). The left column stands for $T_{\text {per }}=15 \tau$, the right one for $T_{\text {per }}=63 \tau$.

row in Table 5.2). As one could expect the attraction to the substrate significantly influences the motion: for smaller attraction the droplet is highly mobile, as the friction is substantially reduced.

Upon increasing the period of vibrations, the CA also increases giving rise to the friction and the frictional dissipations, $T \Sigma_{A}$. The rate of viscous dissipations is not strongly affected by the period of vibrations, as the droplet take the same volume $T \Sigma_{w}$. Additionally, the elasticity of the contact line with its vicinity in $y$ direction, and displacement of liquid particles visible by flux arrows at the CLs give rise to dissipations at CLs, $T \Sigma_{l}$. These dissipations increase with period $T_{\text {per }}$ (cf. the liquid's motion in the vicinity of the receding CL in Table 5.2). As a consequence, the part of the power input left for translational motion of the $\mathrm{CM}$ is smaller at long periods $T_{\text {per }}=25 \tau$ than at shorter ones (cf. Fig. 5.10).

Additionally, we show the velocity field diagrams of droplets at the ASVS of R-type in Table 5.3. The appearance of the table is organized in a similar way, as earlier for the ASVSs of F-type. Having the common features with motion at the R-type substrates 


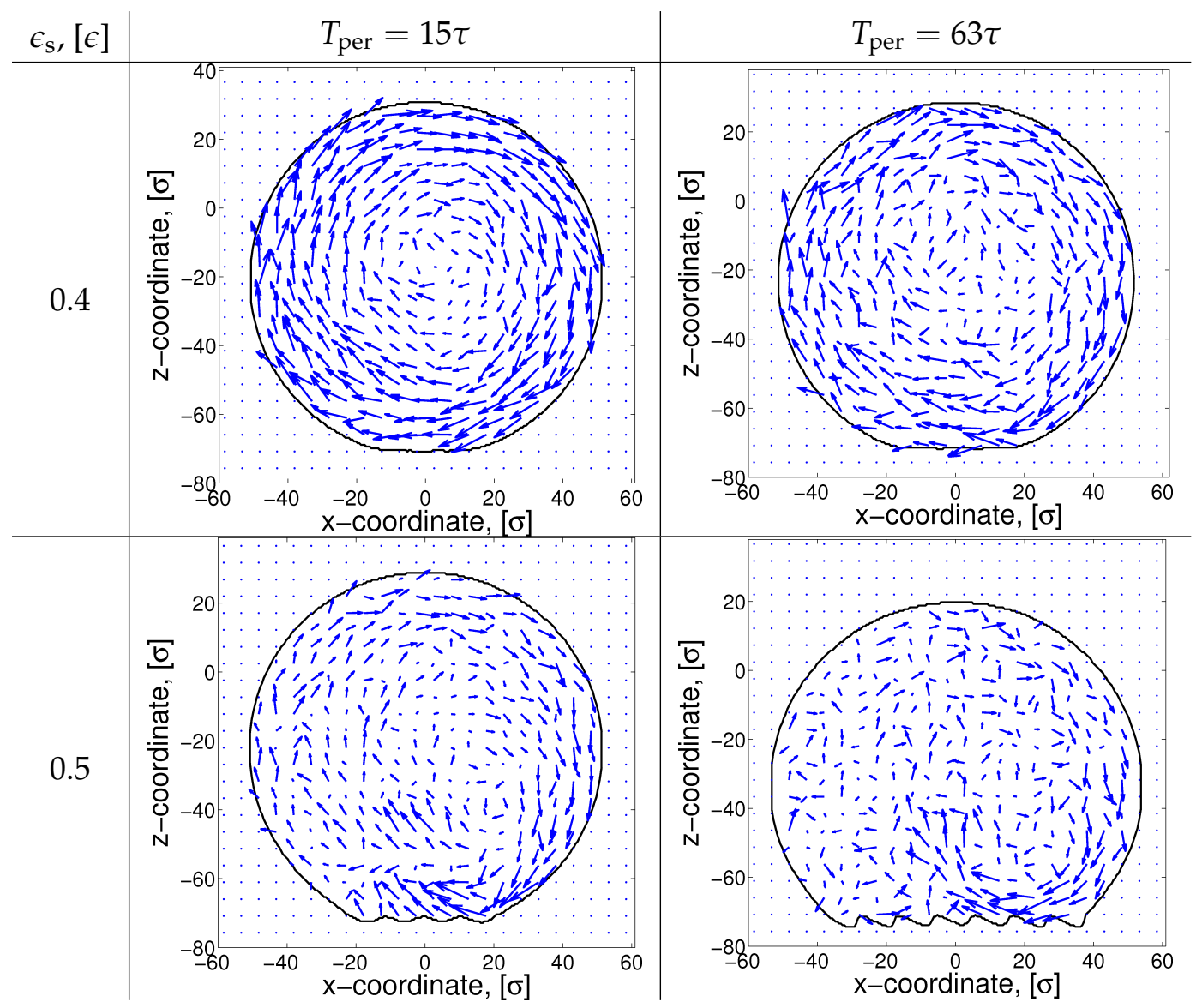

Table 5.3: Velocity fields of moving droplets of $N=200000$ beads at the R-type substrate with solid-liquid interaction strengths $\epsilon_{\mathrm{s}}=0.4 \epsilon$ (top row) and $\epsilon_{\mathrm{s}}=0.5 \epsilon$ (bottom row). The left column stands for $T_{\text {per }}=15 \tau$, the right one for $T_{\text {per }}=63 \tau$.

connected to the strength of solid-liquid interaction $\epsilon_{\mathrm{S}}$ and mobility of the droplet, the motion at the R-type substrate changes significantly. The rotation in clockwise direction is clearly seen at $\epsilon_{\mathrm{s}}=0.4 \epsilon$ and short periods at $\epsilon_{\mathrm{s}}=0.5 \epsilon$. However, the droplet itself is moving to the left. That may be explained accounting for slippage at the solid-liquid boundary. An analogy may be found in the coiled reel on ice pulled by a thread. If the thread is coiled clockwise (i.e. the one pulls the thread touching the reel at the bottom) the reel will rotate clockwise, but because of the slippage will move to the pulling person as a whole.

The shapes of the droplets at $T_{\text {per }}=15 \tau$ and at $\epsilon_{\mathrm{s}}=0.4 \epsilon$ are very close to the perfect cylinder. However, as was discussed, the character of directed motion is different. While in both cases the drops are sliding over the substrate to the left, the surface of the R-type sets up additional rotation to the droplet. Therefore, we conclude that varying topography of the solid one can switch off the rotation or, the other way round, switch it on. The possibility to control the character of droplet's motion may find an application in real microfluidic devices. 


\subsection{Discussion}

In this Chapter, we reported on a realization of the directed transport of droplets at asymmetrically structured and vibrating substrates (ASVSs). At first, we presented an analytical model of the solid-liquid interface movements to explain the direction of motion at ASVSs. The model was verified by the calculation of distances from the substrate to the interface in droplet profiles at different phases of substrate vibrations, by velocity fields diagrams at different phases of vibrations and by the measurement of horizontal components of forces acting on the drop during rising and lowering of the substrate.

Then we investigated the response of the droplets of varying sizes on period of vibrations, and were able to sort out two main mechanisms of driving: along with the driving by the contact lines (CLs), reported in the literature [137, 147], we found that the solid-liquid contact area (CA) itself can drive the droplet additionally to CLs.

The other fundamental problem studied in this Chapter was connected to the character of directed motion and dissipations of input power. It was shown that the roughness of the substrate affects the motion. At a finely corrugated ASVSs the droplet was predominately sliding, whereas at a roughly corrugated ASVSs, an additional steady-state rolling was established. By calculating velocity fields in the droplets we verified the amplification of the frictional dissipations and dissipations at the CLs.

We emphasize, however, that for a better estimation of efficiency of directed motion, the statistics should be significantly improved. The detailed balance between dissipations, however, is a subject to the other profound and detailed investigation. 


\section{CHAPTER 6}

\section{DISCUSSION AND OUTLOOK}

An ambitious target - to build up an entire laboratory set-up on the surface of a labon-a-chip - drives research in micro- and nanofluidics. At present time, such devices experience severe problems due to the limited functionality. However, the future of microelectronics and improvement of standards of life (new textile materials, energy saving and, particularly, water saving) is associated with micro- and, especially, nanodevices. Therefore, more attempts should be devoted to the implementation of ideas on the engineering level.

The aim of this dissertation was to provide a theoretical explanation of the properties and behavior of a small amount of liquid on different kinds of substrates and of the nanoscopic flows in confined geometries. The validity of macroscopic concepts was tested by means of particle-based simulations. This study should by no means be considered as ready-to-real-life-implementation research. However, it gives an insight into wetting phenomena, statics, dynamics and hydrodynamics of micro- and nanoliquids at substrates of different topography. Our observations indicate that the macroscopic concepts cannot be straightforwardly extrapolated down to substrate topographies with dimensions that correspond to tens of fluid particles.

The red line through the dissertation was the topography of the substrate. We started with the simplest one - a flat substrate in Chapter 3 - moved on with symmetrically structured one in Chapter 4 and finished with the most complex, asymmetrically structured substrate in Chapter 5.

In the chapter on flat substrates, a parameter-passing technique was implemented that transfers information from particle-based models based on the phenomenological material constants to the continuum description (CD). The latter has an advantage in direct addressing of engineering time and length scales. A . The liquid-vapor interfacial tension, $\gamma$, and the interface potential $g(h)$ in its dependence on the height of the film were determined by particle-based simulations in the canonical ensemble. We have devised a simple method, which is based on the anisotropy of the pressure inside the film. This general computational strategy can be applied to dense liquids of large macro- 
molecules and can be implemented in standard Molecular Dynamics programs. We particularly stress, that no calculations of chemical potential were needed.

Then, the profiles of drops of varying size obtained by both descriptions (MD and CD) were compared. We found quantitative agreement if (i) the full curvature is used in the continuum model for large contact angles and (ii) the size of the drop is larger than the lateral correlation length, $\xi_{\|}$, of interface fluctuations. We also found that for contact angles up to about $30^{\circ}$ the long-wavelength approximation that is normally used in thin film models describes the droplet shapes even quantitatively quite well. This is an excellent starting point for comparing the dynamics of droplets driven by external forces, which we will pursue in the future.

Additionally, we considered in Chapter 3 hydrodynamic properties of liquid flows confined between flat substrates. It was shown, that applying macroscopic Navier's condition to a microscopic model, two different setups of the flow should be considered: Couette and Poiseuille flows. Then, the solid-liquid interface is described by two material parameters: slip length, $\delta$, and hydrodynamic boundary position, $z_{\mathrm{h}}$. The latter does not necessarily corresponds to the position of the solid-liquid interface, but even in the case of flat walls is located somewhat inside the liquid.

The investigation of properties of liquids at symmetrically structured substrates is discussed in Chapter 4. A technique to estimate the height of the energetic barriers for drops in Cassie and Wenzel states was suggested. It consists in simulations with constraints and quantifies the dependence of the free energy difference on the force applied to the droplet's center of mass to fix it at two close positions. By this method we showed that the height of the energetic barrier increases with strength of solid-liquid interaction $\epsilon_{\mathrm{s}}$, as was initially expected.

To overcome an energetic barrier and set the drop in motion on a corrugated substrate, one has to apply an external force. After some equilibration time, the droplet reaches its steady state and its velocity profile measured across the height of the drop $v_{x}(z)$ allows one to make conclusions about the character of motion. We confirmed predictions of lattice Boltzmann simulations, where the droplet with a contact angle $\theta_{\mathrm{C}} \approx 145^{\circ}$ on a corrugated substrate exhibited a parabolic velocity profile at the substrate that was followed by the linear one further away from it. However, we found that upon increasing the contact angle to $\theta_{\mathrm{C}} \approx 160^{\circ}$, the velocity profile is well approximated by the linear dependence on the distance to the substrate, $z$, across the entire height of the droplet.

Additionally, we incorporated a chessboard corrugation of the solid with the same ratio $\varphi$ of the substrate area covered by posts to the projected area, as in the case of symmetric grooves. It was shown by Moradi et al. [126], that this type of topography can reduce the pinning of the contact line and leads to the higher mobility of the droplet. Indeed, for most of the strengths of solid-liquid interaction $\epsilon_{\mathrm{s}}$, the velocity profiles of 
droplets pushed by a body force over chessboard substrate are somewhat higher than for droplets on symmetric grooves. However, at $\epsilon_{\mathrm{s}}=0.4 \epsilon$, the topography of the substrate did not influence the velocity profile. We suggested that it is explained by an additional friction at the edges of the posts. Therefore, it is not always possible to reduce friction by modification of the substrate's topography.

Then, the flows of a polymer liquid at the coexistence pressure in channels with patterned and flat walls were studied. In applications to superhydrophobic substrates of complex topography the position of the hydrodynamic boundary is not intuitive. Using Couette and Poiseuille flow, we extracted the hydrodynamic boundary position, $z_{\mathrm{h}}$, and slip length, $\delta$, that characterize the Navier slip condition. While the equivalent no-slip plane, which depends on the type of flow, might be located between the top and bottom of the grooves [130], we find that the position of the hydrodynamic boundary, $z_{\mathrm{h}}$, was almost always located above the top of the roughness.

Significant differences between flat and corrugated substrates were found for liquids in the Cassie state. Owing to the friction at the edges of the grooves, however, the friction coefficient $\lambda$ does not scale like the ratio of area covered by the grooves, $\varphi$, to the projected area. For microscopically corrugated substrates, a correction associated with the fiction at the edges of the corrugations is suggested, cf. Eq. 4.15.

Contrary to the expectation, the hydrodynamic properties of the liquid in Wenzel state are not significantly influenced by the substrate corrugation, $r$. For the parameters of our simulation, the difference with respect to a flat substrate is rather controlled by the area fraction $\varphi$, but not the size of the grooves. We showed that there is no sharp phase transition between Cassie and Wenzel states (as expected from macroscopic theory) but only a rather gradual crossover.

Finally, we modeled the propagation of the liquid through a long microfluidic channel with patterned walls accounting for the pressure dependence of the liquid morphology and the concomitant friction. We observed that by using a non-wetting liquid (contact angle on a flat substrate $\theta_{\mathrm{E}}>150^{\circ}$ ) the rate of the frictional dissipation is significantly reduced on the whole length of a long channel in comparison to more wettable substrates. Additionally, we found that only for a liquid at low pressure ( $p<p_{\text {coex }}$ ) the microscopic corrugation affects the hydrodynamic properties, whereas for higher pressures it does not exert a pronounced influence onto the slippage and friction.

At last, we looked at the behavior of nanodroplets at asymmetrically structured vibrating substrates (ASVSs) in Chapter 5. By agitation of the substrate in a harmonic manner we were able to provide the directed transport of droplets.

An analytical model of the solid-liquid interface movements in response to substrate vibration was devised and confirmed by a series of computer experiments. Then, we investigated the nature of the driving mechanisms as a function of the period of vibra- 
tions. Along with the mechanism, reported in the literature [137, 147] (the driving by the contact lines (CLs)), we found another one: additionally to the CLs, the solid-liquid contact area (CA) itself can drive the droplet.

Finally, we showed that the size of corrugation affects the character of directed motion and dissipations of the input power. At a finely corrugated ASVS the droplet was predominately sliding, whereas at a roughly corrugated ASVS, an additional steadystate rolling was established.

We emphasize, however, that the study of droplets on symmetrically and asymmetrically structured substrates is far from completion. At this point, we illuminate several questions crucial for nanodroplets: (i) derivation of a rigorous analytical theory of viscous and frictional dissipations for droplets with high contact angles; (ii) possibilities for reduction of dissipations at the contact lines. Here, one has to account for elastic deformations of the CLs and the displacement of atoms in its vicinity; (iii) proposing of a substrate topography to reach a maximum efficiency of driving. The range of solutions varies from chemically treated substrates to the ones representing randomized corrugations to take advantage of the high contact angles and, simultaneously, of the reduction of an energetic barrier associated with the pinning at the three-phase contact lines. 
In this short Appendix we list the properties of the polymeric chain and of the liquid that are used in previous Chapters. At first we provide configurational properties of the polymer chain. Then, the dynamical properties of the bulk phase are compiled and in the end we define the values of coexistence density and pressure from equation of state (EOS) of the liquid.

\section{Configurational properties of the polymer chain model}

Statistical properties of the chain. We start with the smallest length scale of the polymeric chain - its bond length.

The mean bond length of our chain model is of $b_{0}=0.969 \sigma$.

The short polymer chains consist of $N_{\mathrm{P}}=10$ beads.

The end-to-end radius of the chain is $R_{\mathrm{ee}}=3.43 \sigma$.

\section{Dynamical properties of the polymer liquid}

Dynamic properties of the polymeric liquid were calculated in Ref. [27].

The bulk viscosity is $\eta=5.3 \pm 0.1 \sigma^{2} / \sqrt{m \epsilon}$.

The self-diffusion coefficient $D$ of the polymer liquid at coexistence pressure is $D=$ $0.0157 \pm 0.0030 \sigma^{2} / \tau$.

Thus, the Rouse relaxation time of a polymer is $\tau_{\mathrm{R}}=R_{\mathrm{ee}}^{2} /\left(3 \pi^{2} D\right)$ is $\tau_{\mathrm{R}}=25.3 \pm 5 \tau$.

\section{Equation of state of the polymer liquid}

To calculate the EOS we perform simulations of the polymer liquid confined between two flat walls and measure the density and pressure inside the bulk, while changing the distance between the walls. Taking into account, that vapor pressure is negligible, we find coexistence density $\rho_{\text {coex }}$ providing coexistence pressure $p_{\text {coex }} \approx 0$. By means 


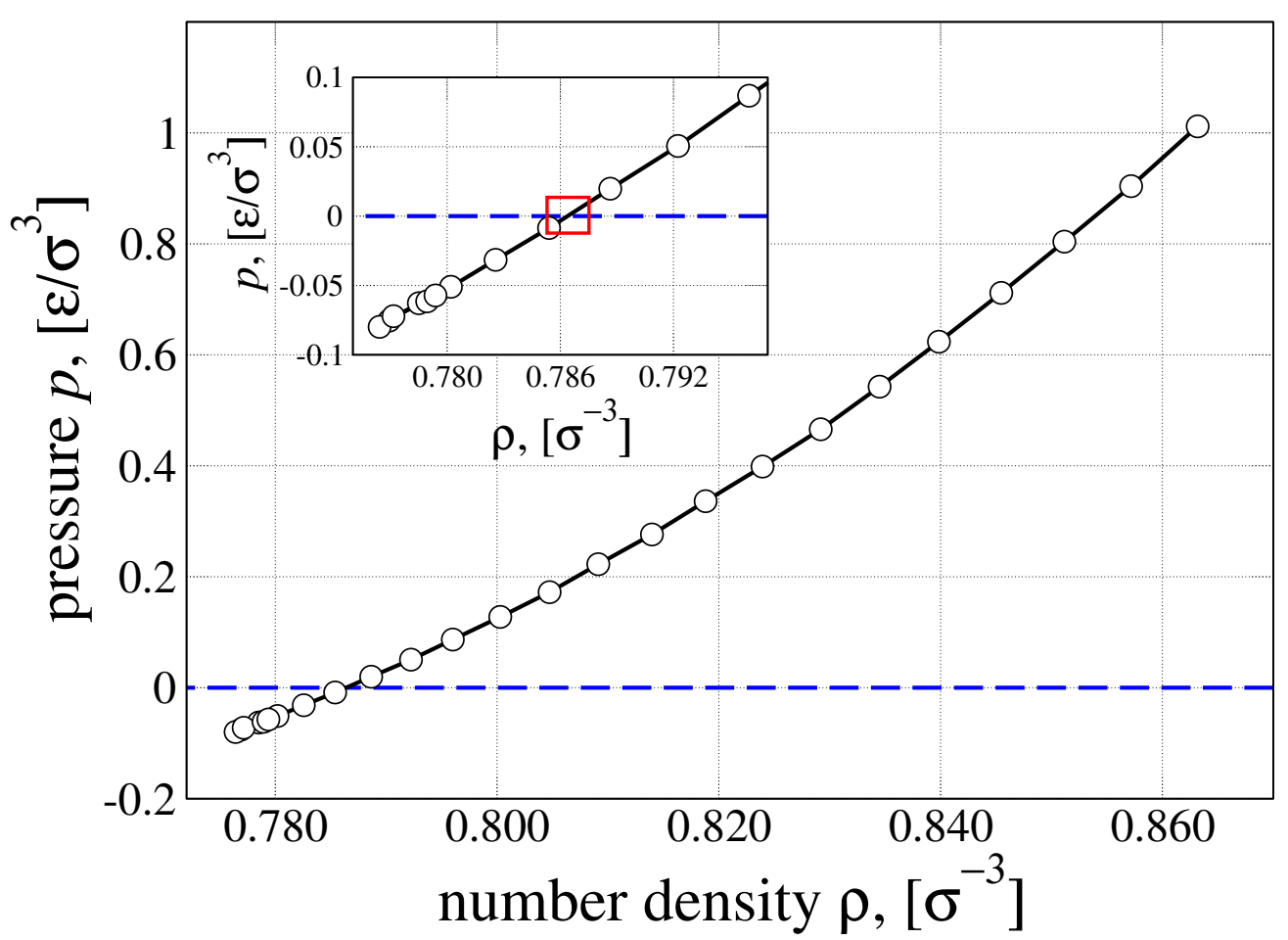

Figure A.1: Equation of state for studied polymeric liquid. The inset zooms into the region of interest. The red square indicates the region, where $p_{\text {coex }} \approx 0$ and $\rho_{\text {coex }} \approx 0.786 \sigma^{-3}$ are defined.

of Fig. A. 1 the coexistence density is found to be $\rho_{\text {coex }} \approx 0.786 \sigma^{-3}$. 


\section{CURRICULUM VITAE}

\section{Personal data}

Name:

Day of birth:

Place of birth:

Nationality:

Address:

\section{Education}

$2003-2009$

March 2009

\section{Nikita Tretyakov}

13th of November 1986

Kaliningrad, Moscow Region, USSR

Russian

Untere-Masch-Str. 20, 37073 Göttingen

Diploma of Physics (Equiv. to M.Sc.) at the Department of Physics of the Lomonosov Moscow State University

Start of the Ph.D. under supervision of Prof. Dr. Marcus Müller at the University of Göttingen 


\section{LIST OF PUBLICATIONS}

- N. Tretyakov, M. Müller, D. Todorova and U. Thiele "Parameter passing between Molecular Dynamics and continuum models for droplets on solid substrates: The static case", submitted to J. Chem. Phys.

- N. Tretyakov and M. Müller "Correlation between surface topography and slippage: a Molecular Dynamics study", submitted to Soft Matter. 


\section{REFERENCES}

[1] F. Durst, An Introduction to the Theory of Fluid Flows. Fluid Mechanics, SpringerVerlag Berlin Heidelberg: Springer, 2010.

[2] T. Young, "An essay on the cohesion of fluids," Phil. Trans. R. Soc. Lond., vol. 95, pp. 65-87, 1805.

[3] P. Mitchell, "Microfluidics-downsizing large-scale biology," Nature Biotechnology, vol. 19 , pp. $717-721,2001$.

[4] H. Bruus, Theoretical Microfluidics. Oxford Master Series in Condensed Matter Physics, Oxford: Oxford Univ. Press, 2008.

[5] S.-W. Lee, D. Y. Kwok, and P. E. Laibinis, "Chemical influences on adsorptionmediated self-propelled drop movement," Phys. Rev. E, vol. 65, p. 051602, 2002.

[6] J. M. K. Ng, I. Gitlin, A. D. Stroock, and G. M. Whitesides, "Components for integrated poly(dimethylsiloxane) microfluidic systems," Electrophoresis, vol. 23, no. 20, pp. 3461-3473, 2002.

[7] G. M. Whitesides, "The origins and the future of microfluidics," Nature, vol. 442, pp. 368-373, 2006.

[8] F. K. Balagaddé, L. You, C. L. Hansen, F. H. Arnold, and S. R. Quake, "Longterm monitoring of bacteria undergoing programmed population control in a microchemostat," Science, vol. 309, no. 5731, pp. 137-140, 2005.

[9] D. B. Weibel, M. Kruithof, S. Potenta, S. K. Sia, A. Lee, and G. M. Whitesides, "Torque-actuated valves for microfluidics," Analytical Chemistry, vol. 77, no. 15, pp. 4726-4733, 2005.

[10] M. Rauscher and S. Dietrich, "Wetting phenomena in nanofluidics," Ann. Rev. Mat. Res., vol. 38, no. 1, pp. 143-172, 2008. 
[11] R. Seemann, M. Brinkmann, T. Pfohl, and S. Herminghaus, "Droplet based microfluidics," Reports on Progress in Physics, vol. 75, no. 1, p. 016601, 2012.

[12] "Hoomd-blue web page, http://codeblue.umich.edu/hoomd-blue."

[13] J. A. Anderson, C. D. Lorenz, and A. Travesset, "General purpose molecular dynamics simulations fully implemented on graphics processing units," J. Comput. Phys., vol. 227, no. 10, pp. 5342 - 5359, 2008.

[14] C. L. Phillips, J. A. Anderson, and S. C. Glotzer, "Pseudo-random number generation for brownian dynamics and dissipative particle dynamics simulations on gpu devices," J. Comput. Phys., vol. 230, no. 19, pp. 7191 - 7201, 2011.

[15] W. Humphrey, A. Dalke, and K. Schulten, "VMD - Visual Molecular Dynamics," Journal of Molecular Graphics, vol. 14, pp. 33-38, 1996.

[16] J. Stone, "An Efficient Library for Parallel Ray Tracing and Animation," Master's thesis, Computer Science Department, University of Missouri-Rolla, 1998.

[17] B. Brooks, R. Bruccoleri, D. Olafson, D. States, S. Swaminathan, and M. Karplus, "Charmm: A program for macromolecular energy, minimization, and dynamics calculations," Journal of Computational Chemistry, vol. 4, pp. 187-217, 1983.

[18] A. MacKerel Jr., C. Brooks III, L. Nilsson, B. Roux, Y. Won, and M. Karplus, CHARMM: The Energy Function and Its Parameterization with an Overview of the Program, vol. 1 of The Encyclopedia of Computational Chemistry, pp. 271-277. John Wiley \& Sons: Chichester, 1998.

[19] W. L. Jorgensen and J. Tirado-Rives, "The opls [optimized potentials for liquid simulations] potential functions for proteins, energy minimizations for crystals of cyclic peptides and crambin," Journal of the American Chemical Society, vol. 110, no. 6, pp. 1657-1666, 1988.

[20] W. D. Cornell, P. Cieplak, C. I. Bayly, I. R. Gould, K. M. Merz, D. M. Ferguson, D. C. Spellmeyer, T. Fox, J. W. Caldwell, and P. A. Kollman, “A second generation force field for the simulation of proteins, nucleic acids, and organic molecules," Journal of the American Chemical Society, vol. 117, no. 19, pp. 5179-5197, 1995.

[21] G. S. Grest and K. Kremer, "Molecular dynamics simulation for polymers in the presence of a heat bath," Phys. Rev. A, vol. 33, pp. 3628-3631, 1986.

[22] M. Murat and K. Kremer, "From many monomers to many polymers: Soft ellipsoid model for polymer melts and mixtures," J. Chem. Phys., vol. 108, no. 10, pp. 4340-4348, 1998. 


\section{REFERENCES}

[23] H. Meyer, O. Biermann, R. Faller, D. Reith, and F. Muller-Plathe, "Coarse graining of nonbonded inter-particle potentials using automatic simplex optimization to fit structural properties," J. Chem. Phys., vol. 113, no. 15, pp. 6264-6275, 2000.

[24] G. Santangelo, A. Di Matteo, F. Müller-Plathe, and G. Milano, "From mesoscale back to atomistic models: A fast reverse-mapping procedure for vinyl polymer chains," The Journal of Physical Chemistry B, vol. 111, no. 11, pp. 2765-2773, 2007.

[25] T. Vettorel, G. Besold, and K. Kremer, "Fluctuating soft-sphere approach to coarse-graining of polymer models," Soft Matter, vol. 6, pp. 2282-2292, 2010.

[26] C. Pastorino, K. Binder, T. Kreer, and M. Müller, "Static and dynamic properties of the interface between a polymer brush and a melt of identical chains," J. Chem. Phys., vol. 124, no. 6, p. 064902, 2006.

[27] J. Servantie and M. Müller, "Statics and dynamics of a cylindrical droplet under an external body force," J. Chem. Phys., vol. 128, no. 1, p. 014709, 2008.

[28] L. G. MacDowell, M. Müller, and K. Binder, "How do droplets on a surface depend on the system size?," Colloids Surf. A: Physicochem. and Eng. Asp., vol. 206, no. 1-3, pp. $277-291,2002$.

[29] O. H. R. B. Bird, R.C. Armstrong, Dynamics of Polymeric Liquids, vol. 1, 2. Wiley, New York, 1977.

[30] K. Kremer and G. S. Grest, "Dynamics of entangled linear polymer melts: A molecular-dynamics simulation," J. Chem. Phys., vol. 92, no. 8, pp. 5057-5086, 1990.

[31] P. J. Hoogerbrugge and J. M. V. A. Koelman, "Simulating microscopic hydrodynamic phenomena with dissipative particle dynamics," Europhys. Lett., vol. 19, no. 3, p. 155, 1992.

[32] P. Español and P. Warren, "Statistical mechanics of dissipative particle dynamics," Europhys. Lett., vol. 30, no. 4, p. 191, 1995.

[33] B. Dünweg and W. Paul, "Brownian dynamics simulations without gaussian random numbers," International Journal of Modern Physics C, vol. 2, no. 3, pp. 817-827, 1991.

[34] F. Léonforte, J. Servantie, C. Pastorino, and M. Müller, "Molecular transport and flow past hard and soft surfaces: Computer simulation of model systems," J. Phys.: Condens. Matter, vol. 23, no. 18, 2011. 


\section{REFERENCES}

[35] B. Leimkuhler, "Comparison of geometric integrators for rigid body simulation.," in Computational Molecular Dynamics: Challenges, Methods, Ideas. (P. Deuflhard, J. Hermans, and B. Leimkuhler, eds.), (Berlin), pp. 349-62, Springer-Verlag, 1999.

[36] W. C. Swope, H. C. Andersen, P. H. Berens, and K. R. Wilson, "A computer simulation method for the calculation of equilibrium constants for the formation of physical clusters of molecules: Application to small water clusters," J. Chem. Phys., vol. 76, no. 1, pp. 637-649, 1982.

[37] H. J. C. Berendsen, Simulating the Physical World. Cambridge University Press, 2007.

[38] L. Verlet, "Computer "experiments" on classical fluids. i. thermodynamical properties of lennard-jones molecules," Phys. Rev., vol. 159, pp. 98-103, 1967.

[39] P. de Gennes, F. Brochard-Wyart, and D. Quéré, Capillarity and Wetting Phenomena: Drops, Bubbles, Pearls, Waves. Springer, 2004.

[40] D. Bhatt, J. Newman, and C. J. Radke, "Molecular simulation of disjoiningpressure isotherms for free liquid, lennard-jones thin films," The Journal of Physical Chemistry B, vol. 106, no. 25, pp. 6529-6537, 2002.

[41] M. Han, "Disjoining properties of Ne and Ar on graphite surface," Colloid Surf. A: Physicochem. Eng. Asp., vol. 317, pp. 679-686, 2008.

[42] M. Allen and D. Tildesley, Computer simulation of liquids. Oxford science publications, Clarendon Press, 1989.

[43] D. Frenkel and B. Smit, Understanding molecular simulation: from algorithms to applications. Computational science, Academic Press, 2002.

[44] J. H. Irving and J. G. Kirkwood, "The statistical mechanical theory of transport processes. iv. the equations of hydrodynamics," J. Chem. Phys., vol. 18, no. 6, pp. 817-829, 1950.

[45] R. C. Tolman, "Consideration of the gibbs theory of surface tension," J. Chem. Phys., vol. 16, no. 8, pp. 758-774, 1948.

[46] F. Varnik, J. Baschnagel, and K. Binder, "Molecular dynamics results on the pressure tensor of polymer films," J. Chem. Phys., vol. 113, no. 10, pp. 4444-4453, 2000.

[47] J. Walton, D. Tildesley, J. Rowlinson, and J. Henderson, "The pressure tensor at the planar surface of a liquid," Mol. Phys., vol. 48, no. 6, pp. 1357-1368, 1983. 


\section{REFERENCES}

[48] P. de Laplace, Théorie de l'action capillaire. Traité de mécanique céleste, Courcier, 1806.

[49] L. A. Girifalco and R. J. Good, "A theory for the estimation of surface and interfacial energies. i. derivation and application to interfacial tension," The Journal of Physical Chemistry, vol. 61, no. 7, pp. 904-909, 1957.

[50] M. Schick, "Introduction in wetting phenomena," in Les Houches Lectures on "Liquids at Interfaces", pp. 415-497, Elsevier Science Publishers BV, Amsterdam, 1990.

[51] M. Müller and K. Binder, "Interface localization-delocalization transition in a symmetric polymer blend: A finite-size scaling Monte Carlo study," Phys. Rev. E, vol. 6302, 2001.

[52] M. Müller and L. G. MacDowell, "Wetting of polymer liquids: Monte carlo simulations and self-consistent field calculations," J. Phys.: Condens. Matter, vol. 15, no. 19, p. R609, 2003.

[53] L. G. MacDowell and M. Müller, "Adsorption of polymers on a brush: Tuning the order of the wetting phase transition," J. Chem. Phys., vol. 124, no. 8, p. 084907, 2006.

[54] E. M. Grzelak and J. R. Errington, "Calculation of interfacial properties via freeenergy-based molecular simulation: The influence of system size," J. Chem. Phys., vol. 132, no. 22, p. 224702, 2010.

[55] A. Vrij, "Possible mechanism for the spontaneous rupture of thin, free liquid films," Discuss. Faraday Soc., vol. 42, pp. 23-33, 1966.

[56] R. Seemann, S. Herminghaus, and K. Jacobs, "Dewetting patterns and molecular forces: A reconciliation," Phys. Rev. Lett., vol. 86, pp. 5534-5537, 2001.

[57] A. Herring and J. Henderson, "Simulation study of the disjoining pressure profile through a three-phase contact line," J. Chem. Phys., vol. 132, p. 084702, 2010.

[58] V. S. Mitlin, "Dewetting of solid surface: Analogy with spinodal decomposition," Journal of Colloid and Interface Science, vol. 156, pp. 491-497, 1993.

[59] U. Thiele, "Thin film evolution equations from (evaporating) dewetting liquid layers to epitaxial growth," J. Phys.: Condens. Matter, vol. 22, p. 084019, 2010.

[60] U. Thiele, M. G. Velarde, K. Neuffer, M. Bestehorn, and Y. Pomeau, “Sliding drops in the diffuse interface model coupled to hydrodynamics," Phys. Rev. E, vol. 64, p. 061601, 2001. 


\section{REFERENCES}

[61] R. Lipowsky and M. E. Fisher, "Scaling regimes and functional renormalization for wetting transitions," Phys. Rev. B., vol. 36, pp. 2126-2141, 1987.

[62] S. Dietrich, Phase Transitions and Critical Phenomena. Academic Press, New York, 1988.

[63] J. Indekeu, "Line tension near the wetting transition: results from an interface displacement model," Physica A: Statistical Mechanics and its Applications, vol. 183, no. 4, pp. $439-461,1992$.

[64] G. Reiter, "Dewetting of thin polymer films," Phys. Rev. Lett., vol. 68, pp. 75-78, 1992.

[65] J. Becker, G. Grün, R. Seemann, H. Mantz, K. Jacobs, K. R. Mecke, and R. Blossey, "Complex dewetting scenarios captured by thin-film models," Nat. Mater., vol. 2, pp. 59-63, 2003.

[66] T. Qian, X.-P. Wang, and P. Sheng, "Molecular scale contact line hydrodynamics of immiscible flows," Phys. Rev. E, vol. 68, p. 016306, 2003.

[67] J. Koplik and J. R. Banavar, "Molecular simulations of dewetting," Phys. Rev. Lett., vol. 84, pp. 4401-4404, 2000.

[68] D. Bonn, J. Eggers, J. Indekeu, J. Meunier, and E. Rolley, "Wetting and spreading," Rev. Mod. Phys., vol. 81, pp. 739-805, 2009.

[69] M. E. R. Shanahan and A. Carre, "Viscoelastic dissipation in wetting and adhesion phenomena," Langmuir, vol. 11, no. 4, pp. 1396-1402, 1995.

[70] M. Müller, C. Pastorino, and J. Servantie, "Flow, slippage and a hydrodynamic boundary condition of polymers at surfaces," J. Phys.: Condens. Matter, vol. 20, no. 49 , p. $494225,2008$.

[71] U. Thiele, M. G. Velarde, K. Neuffer, and Y. Pomeau, "Film rupture in the diffuse interface model coupled to hydrodynamics," Phys. Rev. E, vol. 64, p. 031602, 2001.

[72] B. M. Mognetti, H. Kusumaatmaja, and J. M. Yeomans, "Drop dynamics on hydrophobic and superhydrophobic surfaces," Faraday Discuss., vol. 146, 2010.

[73] A. Milchev and K. Binder, "Polymer melt droplets adsorbed on a solid wall: A monte carlo simulation," J. Chem. Phys., vol. 114, no. 19, pp. 8610-8618, 2001.

[74] J. Koplik, S. Pal, and J. R. Banavar, "Dynamics of nanoscale droplets," Phys. Rev. E, vol. 65, p. 021504, 2002.

[75] D. R. Heine, G. S. Grest, and E. B. Webb, "Spreading dynamics of polymer nanodroplets," Phys. Rev. E, vol. 68, p. 061603, 2003. 


\section{REFERENCES}

[76] J. De Coninck and T. Blake, "Wetting and molecular dynamics simulations of simple liquids," Ann. Rev. Mat. Res., vol. 38, no. 1, pp. 1-22, 2008.

[77] F. Brochard-Wyart, J. M. Di Meglio, D. Quere, and P. G. De Gennes, "Spreading of nonvolatile liquids in a continuum picture," Langmuir, vol. 7, no. 2, pp. 335-338, 1991.

[78] U. Thiele, M. G. Velarde, and K. Neuffer, “Dewetting: Film rupture by nucleation in the spinodal regime," Phys. Rev. Lett., vol. 87, p. 016104, 2001.

[79] A. Dupuis and J. M. Yeomans, "Modeling droplets on superhydrophobic surfaces: equilibrium states and transitions," Langmuir, vol. 21, no. 6, pp. 2624-2629, 2005.

[80] P. G. de Gennes, "Wetting: statics and dynamics," Rev. Mod. Phys., vol. 57, pp. 827-863, 1985.

[81] V. M. Starov and M. G. Velarde, "Surface forces and wetting phenomena," J. Phys.: Condens. Matter, vol. 21, p. 464121, 2009.

[82] V. P. Carey and A. P. Wemhoff, "Disjoining pressure effects in ultra-thin liquid films in micropassages: Comparison of thermodynamic theory with predictions of molecular dynamics simulations," ASME Conference Proceedings, vol. 2005, no. 42223, pp. 511-520, 2005.

[83] J. Koplik and J. R. Banavar, "Continuum deductions from molecular hydrodynamics," Annual Review of Fluid Mechanics, vol. 27, pp. 257-292, 1995.

[84] N. G. Hadjicostantinou, "Combining atomistic and continuum simulations of contact-line motion," Phys. Rev. E, vol. 59, pp. 2475-2478, 1999.

[85] M. Cieplak, J. Koplik, and J. R. Banavar, “Boundary conditions at a fluid-solid interface," Phys. Rev. Lett., vol. 86, pp. 803-806, 2001.

[86] T. Z. Qian, X. P. Wang, and P. Sheng, "Power-law slip profile of the moving contact line in two-phase immiscible flows," Phys. Rev. Lett., vol. 93, p. 094501, 2004.

[87] N. V. Priezjev, A. A. Darhuber, and S. M. Troian, “Slip behavior in liquid films on surfaces of patterned wettability: Comparison between continuum and molecular dynamics simulations," Phys. Rev. E, vol. 71, p. 041608, 2005.

[88] J. O. Indekeu, "Line tension at wetting," International Journal of Modern Physics B, vol. 8, no. 3, pp. 309-345, 1994.

[89] A. Oron, S. H. Davis, and S. G. Bankoff, "Long-scale evolution of thin liquid films," Rev. Mod. Phys., vol. 69, pp. 931-980, 1997. 


\section{REFERENCES}

[90] U. Thiele, "Structure formation in thin liquid films," in Thin films of Soft Matter (S. Kalliadasis and U. Thiele, eds.), (Wien), pp. 25-93, Springer, 2007.

[91] E. Doedel, H. B. Keller, and J. P. Kernevez, "Numerical analysis and control of bifurcation problems (I) Bifurcation in finite dimensions," Int. J. Bifurcation Chaos, vol. 1, pp. 493-520, 1991.

[92] D. Todorova, U. Thiele, and L. Pismen, "The relation of steady evaporating drops fed by an influx and freely evaporating drops," J. Eng. Math., vol. 73, pp. 17-30, 2012.

[93] A. Sharma, "Equilibrium contact angles and film thicknesses in the apolar and polar systems: Role of intermolecular interactions in coexistence of drops with thin films," Langmuir, vol. 9, p. 3580, 1993.

[94] V. M. Starov, "Equilibrium and hysteresis contact angles," Adv. Colloid Interface Sci., vol. 39, pp. 147-173, 1992.

[95] G. O. Berim and E. Ruckenstein, "Nanodrop on a nanorough solid surface: Density functional theoryconsiderations," J. Chem. Phys., vol. 129, no. 1, p. 014708, 2008.

[96] D. Abraham, F. Latremoliere, and P. Upton, "Divergence of the point tension at wetting," Phys. Rev. Lett., vol. 71, no. 3, pp. 404-407, 1993.

[97] A. Hariharan and J. G. Harris, "Structure and thermodynamics of the liquidvapor interface of fluorocarbons and semifluorinated alkane diblocks: A molecular dynamics study," J. Chem. Phys., vol. 101, no. 5, pp. 4156-4165, 1994.

[98] J. L. Rivera, C. McCabe, and P. T. Cummings, "Molecular simulations of liquidliquid interfacial properties: Water- $n$-alkane and water-methanol- $n$-alkane systems," Phys. Rev. E, vol. 67, p. 011603, 2003.

[99] L. D. Landau and E. M. Lifshitz, Fluid Mechanics, Second Edition: Volume 6 (Course of Theoretical Physics). Butterworth-Heinemann, 2 ed., 1987.

[100] J. P. Rothstein, "Slip on superhydrophobic surfaces," Annual Review of Fluid Mechanics, vol. 42, no. 1, pp. 89-109, 2010.

[101] O. Bäumchen and K. Jacobs, "Slip effects in polymer thin films," J. Phys.: Condens. Matter, vol. 22, no. 3, p. 033102, 2010.

[102] O. I. Vinogradova and A. V. Belyaev, "Wetting, roughness and flow boundary conditions," J. Phys.: Condens. Matter, vol. 23, no. 18, p. 184104, 2011. 


\section{REFERENCES}

[103] C. L. M. H. Navier, "Mémoire sur les lois du mouvement des fluids," Mem. Acad. Sci. Inst. Fr., vol. 6, pp. 432-6, 1823.

[104] J.-L. Barrat and L. Bocquet, "Large slip effect at a nonwetting fluid-solid interface," Phys. Rev. Lett., vol. 82, pp. 4671-4674, 1999.

[105] J.-L. Barrat and L. Bocquet, "Influence of wetting properties on hydrodynamic boundary conditions at a fluid/solid interface," Faraday Discuss., vol. 112, pp. 119-128, 1999.

[106] M. Müller and C. Pastorino, "Cyclic motion and inversion of surface flow direction in a dense polymer brush under shear," Europhys. Lett., vol. 81, no. 2, p. 28002, 2008.

[107] J. Smiatek, M. Allen, and F. Schmid, "Tunable-slip boundaries for coarse-grained simulations of fluid flow," The European Physical Journal E: Soft Matter and Biological Physics, vol. 26, pp. 115-122, 2008.

[108] L. Bocquet and J.-L. Barrat, "Flow boundary conditions from nano- to microscales," Soft Matter, vol. 3, 2007.

[109] C. Cottin-Bizonne, S. Jurine, J. Baudry, J. Crassous, F. Restagno, and E. Charlaix, "Nanorheology: An investigation of the boundary condition at hydrophobic and hydrophilic interfaces," The European Physical Journal E: Soft Matter and Biological Physics, vol. 9, pp. 47-53, 2002.

[110] C. Cottin-Bizonne, J.-L. Barrat, L. Bocquet, and E. Charlaix, "Low-friction flows of liquid at nanopatterned interfaces," Nat. Mater., vol. 2, pp. 237-240, 2003.

[111] S. Granick, Y. Zhu, and H. Lee, "Slippery questions about complex fluids flowing past solids," Nat. Mater., vol. 2, no. 4, pp. 221-227, 2003.

[112] C. Cottin-Bizonne, C. Barentin, E. Charlaix, L. Bocquet, and J. Barrat, “Dynamics of simple liquids at heterogeneous surfaces: Molecular-dynamics simulations and hydrodynamic description," The European Physical Journal E: Soft Matter and Biological Physics, vol. 15, pp. 427-438, 2004.

[113] C. Lee, C.-H. Choi, and C.-J. C. Kim, "Structured surfaces for a giant liquid slip," Phys. Rev. Lett., vol. 101, p. 064501, 2008.

[114] S. Schmieschek, A. V. Belyaev, J. Harting, and O. I. Vinogradova, “Tensorial slip of superhydrophobic channels," Phys. Rev. E, vol. 85, p. 016324, 2012.

[115] T. Qian, X.-P. Wang, and P. Sheng, "Hydrodynamic slip boundary condition at chemically patterned surfaces: A continuum deduction from molecular dynamics," Phys. Rev. E, vol. 72, p. 022501, 2005. 
[116] N. V. Priezjev, "Molecular diffusion and slip boundary conditions at smooth surfaces with periodic and random nanoscale textures," J. Chem. Phys., vol. 135, no. 20, p. 204704, 2011.

[117] J. Servantie and M. Müller, “Temperature dependence of the slip length in polymer melts at attractive surfaces," Phys. Rev. Lett., vol. 101, pp. 4989-5001, 2008.

[118] R. N. Wenzel, "Resistance of solid surfaces to wetting by water," Industrial $\mathcal{E}$ Engineering Chemistry, vol. 28, no. 8, pp. 988-994, 1936.

[119] A. B. D. Cassie and S. Baxter, "Wettability of porous surfaces," Trans. Faraday Soc., vol. 40, pp. 546-551, 1944.

[120] H. Kusumaatmaja, C. M. Pooley, S. Girardo, D. Pisignano, and J. M. Yeomans, “Capillary filling in patterned channels," Phys. Rev. E, vol. 77, p. 067301, 2008.

[121] M. Gross, F. Varnik, and D. Raabe, "Fall and rise of small droplets on rough hydrophobic substrates," Europhys. Lett., vol. 88, no. 2, p. 26002, 2009.

[122] M. Gross, F. Varnik, D. Raabe, and I. Steinbach, "Small droplets on superhydrophobic substrates," Phys. Rev. E, vol. 81, p. 051606, 2010.

[123] J. Bico, C. Marzolin, and D. Quéré, "Pearl drops," Europhys. Lett., vol. 47, no. 2, p. 220, 1999.

[124] D. Quéré, A. Lafuma, and J. Bico, "Slippy and sticky microtextured solids," Nanotechnology, vol. 14, no. 10, p. 1109, 2003.

[125] L. Mahadevan and Y. Pomeau, "Rolling droplets," Physics of Fluids, vol. 11, no. 9, pp. 2449-2453, 1999.

[126] N. Moradi, F. Varnik, and I. Steinbach, "Roughness-gradient-induced spontaneous motion of droplets on hydrophobic surfaces: A lattice boltzmann study," Europhys. Lett., vol. 89, no. 2, p. 26006, 2010.

[127] D. Herde, U. Thiele, S. Herminghaus, and M. Brinkmann, "Driven large contact angle droplets on chemically heterogeneous substrates," Europhysics Letters (EPL), vol. 100, 2012.

[128] J. Ou, B. Perot, and J. P. Rothstein, "Laminar drag reduction in microchannels using ultrahydrophobic surfaces," Physics of Fluids, vol. 16, no. 12, pp. 4635-4643, 2004.

[129] M. Queralt-Martín, M. Pradas, R. Rodríguez-Trujillo, M. Arundell, E. Corvera Poiré, and A. Hernández-Machado, "Pinning and Avalanches in Hydrophobic Microchannels," Phys. Rev. Lett., vol. 106, no. 19, p. 194501, 2011. 


\section{REFERENCES}

[130] C. Kunert, J. Harting, and O. I. Vinogradova, "Random-roughness hydrodynamic boundary conditions," Phys. Rev. Lett., vol. 105, p. 016001, 2010.

[131] A. O. Parry, C. Rascon, N. B. Wilding, and R. Evans, "Condensation in a capped capillary is a continuous critical phenomenon," Phys. Rev. Lett., vol. 98, no. 22, p. 226101, 2007.

[132] R. Roth and A. O. Parry, "Drying in a capped capillary," Mol. Phys., vol. 109, no. 7-10, pp. 1159-1167, 2011.

[133] C. Y. Soong, T. H. Yen, and P. Y. Tzeng, "Molecular dynamics simulation of nanochannel flows with effects of wall lattice-fluid interactions," Phys. Rev. E, vol. 76, p. 036303, 2007.

[134] A. Steinberger, C. Cottin-Bizonne, P. Kleimann, and E. Charlaix, "High friction on a bubble mattress," Nat. Mater., vol. 6, no. 9, pp. 665-668, 2007.

[135] O. Vinogradova, N. Bunkin, N. Churaev, O. Kiseleva, A. Lobeyev, and B. Ninham, "Submicrocavity structure of water between hydrophobic and hydrophilic walls as revealed by optical cavitation," Journal of Colloid and Interface Science, vol. 173, no. 2, pp. $443-447,1995$.

[136] A. Shastry, M. J. Case, and K. F. Böhringer, "Directing droplets using microstructured surfaces," Langmuir, vol. 22, no. 14, pp. 6161-6167, 2006.

[137] M. Reyssat, F. Pardo, and D. Quéré, "Drops onto gradients of texture," Europhys. Lett., vol. 87, no. 3, p. 36003, 2009.

[138] M. K. Chaudhury and G. M. Whitesides, "How to make water run uphill," Science, vol. 256, no. 5063, pp. 1539-1541, 1992.

[139] F. Mugele and J.-C. Baret, "Electrowetting: from basics to applications," J. Phys.: Condens. Matter, vol. 17, no. 28, p. R705, 2005.

[140] F. D. Dos Santos and T. Ondarçuhu, "Free-running droplets," Phys. Rev. Lett., vol. 75, pp. 2972-2975, 1995.

[141] F. Brochard, "Motions of droplets on solid surfaces induced by chemical or thermal gradients," Langmuir, vol. 5, no. 2, pp. 432-438, 1989.

[142] H. Kusumaatmaja, J. Léopoldès, A. Dupuis, and J. M. Yeomans, "Drop dynamics on chemically patterned surfaces," Europhys. Lett., vol. 73, no. 5, p. 740, 2006.

[143] S. Daniel and M. K. Chaudhury, "Rectified motion of liquid drops on gradient surfaces induced by vibration," Langmuir, vol. 18, no. 9, pp. 3404-3407, 2002. 


\section{REFERENCES}

[144] A. Buguin, L. Talini, and P. Silberzan, "Ratchet-like topological structures for the control of microdrops," Applied Physics A: Materials Science E Processing, vol. 75, pp. 207-212, 2002.

[145] K. John and U. Thiele, "Liquid transport generated by a flashing field-induced wettability ratchet," Applied Physics Letters, vol. 90, no. 26, p. 264102, 2007.

[146] K. John and U. Thiele, "Self-ratcheting stokes drops driven by oblique vibrations," Phys. Rev. Lett., vol. 104, p. 107801, 2010.

[147] M. Prakash, D. Quéré, and J. W. M. Bush, "Surface tension transport of prey by feeding shorebirds: The capillary ratchet," Science, vol. 320, no. 5878, pp. 931-934, 2008.

[148] J. Maxwell, Theory of Heat (1871). Dover Books on Physics Series, Dover Publications, 2001 ed., 1871.

[149] J. F. Joanny and P. G. de Gennes, "A model for contact angle hysteresis," J. Chem. Phys., vol. 81, no. 1, pp. 552-562, 1984.

[150] T. D. Blake, "The physics of moving wetting lines," Journal of Colloid and Interface Science, vol. 299, no. 1, pp. 1 -13, 2006. 